\title{
Optimal Reporting Systems with Investor Information Acquisition
}

\author{
by \\ Zeqiong Huang \\ Department of Business Administration \\ Duke University \\ Date: \\ Approved: \\ Qi Chen, Supervisor \\ S. Viswanathan, Co-Supervisor \\ \begin{tabular}{c}
\hline Frank Ecker \\
\hline $\mathrm{Xu}$ Jiang
\end{tabular}
}

Katherine Schipper

Rahul Vashishtha

Dissertation submitted in partial fulfillment of the requirements for the degree of

Doctor of Philosophy in the Department of Business Administration in the Graduate School of Duke University

2016 


\title{
$\underline{\text { ABSTRACT }}$ \\ Optimal Reporting Systems with Investor Information Acquisition
}

\author{
by \\ Zeqiong Huang \\ Department of Business Administration \\ Duke University
}

Date:

Approved:

Qi Chen, Supervisor

S. Viswanathan, Co-Supervisor

Frank Ecker

\begin{tabular}{c}
\hline Xu Jiang \\
\hline Katherine Schipper
\end{tabular}

Rahul Vashishtha

An abstract of a dissertation submitted in partial fulfillment of the requirements for the degree of Doctor of Philosophy in the Department of Business Administration in the Graduate School of Duke University 2016 
Copyright (c) 2016 by Zeqiong Huang All rights reserved except the rights granted by the Creative Commons Attribution-Noncommercial Licence 


\section{Abstract}

This paper analyzes a manager's optimal ex-ante reporting system using a Bayesian persuasion approach (Kamenica and Gentzkow (2011)) in a setting where investors affect cash flows through their decision to finance the firm's investment opportunities,

possibly assisted by the costly acquisition of additional information (inspection). I examine how the informativeness and the bias of the optimal system are determined by investors' inspection cost, the degree of incentive alignment between the manager and investors, and the prior belief that the project is profitable. I find that a misaligned manager's system is informative only when the market prior is pessimistic and is always positively biased. As investors' inspection cost decreases, this bias decreases and the optimal system becomes more conservative. In contrast, a wellaligned manager's system is fully revealing when investors' inspection cost is high, and is counter-cyclical to the market belief when the inspection cost is low: It is positively (negatively) biased when the market belief is pessimistic (optimistic). I explore the extent to which the results generalize to a case with managerial manipulation and discuss the implications for investment efficiency. Overall, the analysis describes the complex interactions among determinants of firm disclosures and governance, and offers explanations for the mixed empirical results in this area. 


\section{Contents}

Abstract $\quad$ iv

List of Tables viii

List of Figures $\quad$ ix

List of Abbreviations and Symbols $\quad \mathrm{x}$

Acknowledgements $\quad$ xii

1 Introduction 1

1.1 Related literature . . . . . . . . . . . . . . . . . . . 8

2 The Basic Model $\quad 11$

2.1 The firm and the manager . . . . . . . . . . . . . . 11

2.2 Disclosure strategy as the design of a reporting system . . . . . . . . 12

2.3 The investor . . . . . . . . . . . . . . . . . . . . . . . . . . 15

3 Optimal Reporting Systems $\quad 17$

3.1 Investor's reaction to disclosure . . . . . . . . . . . . . . . . . . 17

3.2 The manager's problem of designing a reporting system . . . . . . . . 20

3.3 Mis-aligned manager's optimal reporting system . . . . . . . . . . . . 22

3.3.1 Mis-aligned manager and high investor inspection costs . . . . 23

3.3.2 Mis-aligned manager and low investor inspection costs . . . . 26

3.4 Well-aligned manager's optimal reporting system _. . . . . . . . . 30

3.4.1 Well-aligned manager and high investor inspection costs . . . 31 
3.4.2 Well-aligned manager and low investor inspection costs . . . . 32

3.5 Further discussions . . . . . . . . . . . . . . . . . 36

3.5.1 Private information about the manager's incentives . . . . . . 37

3.5.2 Private information when designing the reporting system . . . 37

3.5.3 Heterogeneous priors . . . . . . . . . . . . . . 39

3.5.4 Alternative payoff/cost functions of investor inspection . . . . 39

3.5.5 The manager internalizes the inspection cost . . . . . . . . . 40

3.5.6 Variable investment . . . . . . . . . . . . . . . . 41

3.5.7 Alternative timeline: investor inspection and manager's choice are simultaneous . . . . . . . . . . . . . . . . . . 41

4 The Extended Model $\quad 43$

4.1 Model Setup . . . . . . . . . . . . . . . . . 44

4.2 The equilibrium . . . . . . . . . . . . . . . . . 45

4.3 Comparison with the no-manipulation case . . . . . . . . . . 45

4.4 Well-aligned manager with ex-post manipulation . . . . . . . . . 46

4.5 Mis-aligned manager with ex-post manipulation . . . . . . . . . . . 47

4.5.1 Mis-aligned manager and high investor inspection costs . . . . 47

4.5.2 Mis-aligned manager and low investor inspection costs . . . . 49

5 Empirical Implications $\quad 53$

6 Conclusion $\quad 58$

$\begin{array}{ll}\text { A Proofs } & 60\end{array}$

A.1 Proof of Lemma 1 . . . . . . . . . . . . . . . 60

A.2 Proof of Proposition $1 \ldots \ldots \ldots$. . . . . . . . . . . 61

A.3 Proof of Corollary 1. . . . . . . . . . . . . . . 64

A.4 Proof of Corollary 2 . . . . . . . . . . . . . . . 65

A.5 Proof of Proposition $2 \ldots \ldots \ldots$. . . . . . . . . . . . 65 
A.6 Proof of Corollary $3 \ldots \ldots$. . . . . . . . . . . . . . . . . 67

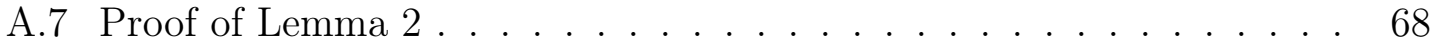

A.8 Proof of Lemma $3 \ldots \ldots \ldots$. . . . . . . . . . . . . . . . . . . . 69

A.9 Proof of Lemma $4 \ldots \ldots \ldots$. . . . . . . . . . . . . 70

A.10 Proof of Lemma $5 \ldots \ldots \ldots$. . . . . . . . . . . . . . 71

A.11 Lemma A1 and its proof . . . . . . . . . . . . . . 71

A.12 Lemma A2 and its proof . . . . . . . . . . . . . . . 72

A.13 Lemma A3 and its proof . . . . . . . . . . . . . . 74

A.14 Proof of Proposition $3 \ldots \ldots \ldots$. . . . . . . . . 76

A.15 Lemma A4 and its proof . . . . . . . . . . . . . . 77

A.16 Proof of Proposition $4 \ldots \ldots$. . . . . . . . . . . . . . 81

A.17 Proof of Corollary 4 . . . . . . . . . . . . . . . . . . 81

A.18 Proof of Corollary 5 . . . . . . . . . . . . . . . . 82

A.19 Proof of Corollary $6 \ldots \ldots \ldots$. . . . . . . . . . . . 82

B Summary of Results $\quad 84$

$\begin{array}{lr}\text { Bibliography } & 89\end{array}$

$\begin{array}{ll}\text { Biography } & 95\end{array}$ 


\section{List of Tables}

B.1 The Mis-aligned Manager's Optimal System in the Basic Model . . . 85

B.2 The Well-aligned Manager's Optimal System in the Basic Model . . . 86

B.3 The Mis-aligned Manager's Optimal System in the Extended Model . 87

B.4 The Well-aligned Manager's Optimal System in the Extended Model 88 


\section{List of Figures}

2.1 The Reporting System in the Basic Model . . . . . . . . . . . . . . . 13

2.2 The Timeline in the Basic Model . . . . . . . . . . . . . . . . . . . . 16

3.1 The Investor's Optimal Action . . . . . . . . . . . . . . . . . . . . . . 19

3.2 The Mis-aligned Manager's Expected Payoff . . . . . . . . . . . . . . 23

3.3 The Mis-aligned Manager's Optimal System . . . . . . . . . . . . . 28

3.4 The Well-aligned Manager's Expected Payoff . . . . . . . . . . . . . . 31

3.5 The Well-aligned Manager's Optimal System . . . . . . . . . . . . . . 33

3.6 Optimal Reporting Systems for Mis-aligned and Well-aligned Managers 36

4.1 The Reporting System in the Extended Model . . . . . . . . . . . . . 44

4.2 The Timeline in the Extended Model . . . . . . . . . . . . . . . . . 44

4.3 Properties of the Optimal Reporting System when Manipulation is Allowed .......................... 49 


\title{
List of Abbreviations and Symbols
}

\author{
Symbols in the Basic Model
}

$N_{g}, N_{b} \quad$ The net present value of the project in the good and bad states, respectively.

$\mu_{0} \quad$ The commonly known prior that the state of the project (firm) is good.

$\lambda_{g}, \lambda_{b} \quad$ The probability that the reporting system maps the good or bad state $\omega \in\{g, b\}$ into a high signal $h$.

$s \quad$ The manager's share of equity.

$m \quad$ The investor inspection cost.

$B \quad$ The manager's private benefit from an approved project.

$k \quad$ The cost of the reporting system per unit of entropy reduction.

$u(\mu, a) \quad$ The investor's expected payoff when her belief that the state is good is $\mu$ and her action is $a \in\{A, I, R\}$.

$\mu_{h}, \mu_{l} \quad$ The investor's posterior belief that the state is good after observing the signal $h$ or $l$, respectively.

$\bar{m} \quad$ The threshold of investor inspection cost above which the investor never finds inspection optimal.

$\bar{\mu}_{1} \quad$ The threshold belief above which the investor prefers inspection, compared to outright rejection; $\bar{\mu}_{1}=\frac{m}{(1-s) N_{g}}$.

$\bar{\mu}_{2} \quad$ The threshold belief above which the investor prefers outright approval, compared to inspection; $\bar{\mu}_{2}=1-\frac{m}{(1-s)\left|N_{b}\right|}$.

$\hat{v}_{c}\left(\mu_{0}\right) \quad$ The manager's expected payoff conditional on belief $\mu_{0}$ when the reporting system is costly $(k>0) . \quad \hat{v}_{c}\left(\mu_{0}\right)=\hat{v}\left(\mu_{0}\right)-$ $k\left(H\left(\mu_{r}\right)-H\left(\mu_{\pi}\left(\mu, \mu_{r}\right)\right)\right)$. 
$\hat{v}\left(\mu_{0}\right) \quad$ The manager's expected payoff at $\mu_{0}$ when the reporting system cost approaches $0\left(k \rightarrow 0^{+}\right)$.

$v_{F I}\left(\mu_{0}\right) \quad$ The manager's expected payoff at $\mu_{0}$ under a fully revealing reporting system.

$V\left(\mu_{0}\right) \quad$ The manager's maximum expected payoff at $\mu_{0}$ when $k \rightarrow 0^{+}$.

\section{Additional Symbols in the Extended Model}

$\delta \quad$ The scope of manipulation: If the manager manipulates, he alters the final output of the reporting system with probability $\delta$.

F The fixed cost of manipulating the reporting system.

$\chi_{h^{\prime}}, \chi_{l^{\prime}} \quad$ The probability that the investor approves outright upon observing a $h^{\prime}\left(l^{\prime}\right)$ signal.

$\phi_{h}, \phi_{l} \quad$ The equilibrium probability that a manager manipulates the system upon observing a $h(l)$ output.

$\mu_{y^{\prime}}\left(\phi_{h}, \phi_{l}\right) \quad$ The posterior belief that the state is good, after observing the signal $h^{\prime}$ or $l^{\prime}$, when the $h$-signal (l-signal) manager manipulates with probability $\phi_{h}\left(\phi_{l}\right)$.

$V_{\delta}\left(\mu_{0}\right) \quad$ The manager's maximum expected payoff at $\mu_{0}$, when the scope of manipulation is $\delta$. 


\section{Acknowledgements}

I would like to express my deepest appreciation to my dissertation committee members, Qi Chen, S. "Vish" Viswanathan, Frank Ecker, Xu Jiang, Katherine Schipper and Rahul Vashishtha, for their continuous guidance and support. I learned tremendously from them about how to conduct research and how to contribute to the profession. I am deeply enlightened by their work and teaching, and both influenced and inspired by their immense knowledge and enormous enthusiasm for research. Without their encouragement and patience, this dissertation would not be possible.

I greatly appreciate valuable comments from Jeremy Bertomeu, Scott Dyreng, Shane Dikolli, Giuseppe (Pino) Lopomo, Bill Mayew, Mohan Venkatachalam. I also thank Shiming Fu, Thomas Steffen, Alex Young, Mani Sethuraman, Huihui Wang, Christopher Calvin, Huihao Yan, and other Ph.D. colleagues for helpful conversations.

I benefited greatly from helpful comments from workshop participants at Duke University, University of Chicago, University of Minnesota, Pennsylvania State University, Purdue University, Stanford University and Yale University.

Last but not least, I thank my family for their love and support throughout my life, and thank all my friends who encouraged me to strive towards my goal. 


\section{Introduction}

This dissertation analyzes firms' ex-ante optimal reporting systems in a stylized setting in which reported information can affect investor's subsequent decisions to acquire additional information and to approve (finance) firms' future investments, which ultimately alters firms' cash flows. ${ }^{1}$ The primary purpose is to understand how managers optimally choose strategies for reporting forward-looking information about future profitability when that information affects subsequent investments through affecting investors' reactions to these disclosures. To answer this question, my model relaxes three assumptions in the prior theoretical literature. The first assumption is that investors (shareholders) use the disclosed information either for stewardship purposes, i.e., to assess managers' past performance and determine their compensation (e.g., Gigler and Hemmer (2001)), or for passive valuation purposes, i.e., to estimate the firm's exogenously given cash flows (e.g., Dye (1985), Bertomeu

${ }^{1}$ I use reporting system, reporting strategy, and disclosure strategy interchangeably. All refer to managers' discretionary choices that affect the amount and nature of information released to investors. In the context of mandatory periodic reporting, managers are often permitted discretion in choosing accounting methods and estimates which can affect investors' assessment of the profitability of firms' future investments. In the context of voluntary disclosure, managers exercise significant discretion in whether to disclose certain information and how informative the disclosure is. 
et al. (2011)). In both cases, the investors' decision after receiving the disclosure does not affect firms' future investment decisions and cash flows. Second, investors rely solely on firm information for their decision-making without actively seeking additional private information. ${ }^{2}$ Third, managers' disclosure strategy is executed ex post, contingent on the private information they receive exogenously. Managers choose whether to disclose (e.g., Jung and Kwon (1988)) or to take real actions to signal their private information (e.g., Kanodia and Lee (1998), Beyer and Guttman (2012))

I show that relaxing these assumptions generates predictions that speak to a large body of empirical findings, and provides new insights on how several commonly discussed factors, such as managerial incentive misalignment and investors' prior beliefs, jointly shape firms' reporting behaviors. Specifically, I relax the first assumption to highlight that firm disclosures often contain forward-looking elements useful for investors to make decisions that affect firms' future operations, ${ }^{3}$ and that such decisions affect managers' future payoffs. Most disclosures fit this description, including both voluntary disclosures of forward-looking information and mandatory reports of past performance that investors use to assess firms' future profitability. ${ }^{4}$ I relax the second assumption to describe more realistically how investors collect information prior

${ }^{2}$ Dye and Sridhar (2002) and Gao and Liang (2013) explicitly consider investors' information acquisition, but investors do not directly affect firms' investment decisions in their models.

3 This decision-making view is broadly consistent with the perspective of standard setters as expressed in Statement of Financial Accounting Concepts No. 8: "General purpose financial reports are not designed to show the value of a reporting entity; but they provide information to help existing and potential investors, lenders, and other creditors to estimate the value of the reporting entity." (FASB (2010)). While the conceptual framework does not specify how investors estimate firm values, in practice, investors often do so by relying on financial statements to evaluate the profitability of firms' future investment plans.

${ }^{4}$ In fact, viewing firms as going concerns whose values largely depend on future decisions and the outcomes of these decisions, a significant number of disclosures are potentially useful for assessing firms' future. Potentially useful does not necessarily mean observed disclosures actually move price or change investors' priors. In fact, in my model, whether observed disclosure changes investors' prior beliefs is the outcome of the endogenous choice by managers on the informativeness of the disclosures. 
to decision-making. Relaxing this assumption also enables me to address the important and increasingly pertinent question of how the reporting properties of reporting systems are affected by investors' costs to acquire additional information, which have arguably been significantly reduced by computational and communication technology (see Magee (2001), Healy and Palepu (2001)). ${ }^{5}$ I relax the third assumption of ex-post disclosure (i.e., the manager's disclosure behavior is contingent on his exogenously endowed private information) by examining firms' disclosure strategy from an ex-ante perspective. This perspective is suitable to study the disclosure of forward-looking information, for which information asymmetry between managers and investors is less likely. In addition, examining the ex-ante strategy also allows me to endogenize both the informativeness and the bias of disclosed information. ${ }^{6}$

My model describes a firm with a potential investment project that requires a representative investor's approval to proceed. The project's payoff is uncertain and depends on the underlying state of the world. The state can be either good or bad, and the project has a positive net present value (NPV) only when the state is good. Neither the firm nor the investor observes the true state, and their uncertainty is captured by a common prior belief. In addition, the firm has an information system that maps each possible state (good or bad) into a binary signal (high or low) to be released to the investor. Importantly, after observing the firm's reported signal, but prior to making her approval decision, the investor has the option to acquire additional information, at her own expense, that would reveal the true state.

${ }^{5}$ The Financial Accounting Standards Board acknowledges this possibility in Statement of Financial Accounting Concepts No. 8: "if needed information is not provided, users incur additional costs to obtain that information elsewhere or to estimate it" (FASB (2010)). Magee (2001) called for more research on this issue: "... a common denominator in these questions [is] uncertainty about the role of accounting measurement when the economics of information delivery are changing rapidly."

${ }^{6}$ Prior literature has also studied ex-ante disclosure strategies (e.g., Diamond and Verrecchia (1991) and Gao and Liang (2013)). However, these papers restrict the information structure (e.g., to choosing the precision of an additive, normally-distributed noise term, or to choosing the probability of disclosure), and thus cannot endogenize both informativeness and bias. 
I model the firm's reporting strategy as the manager designing the mapping rule governing how the reporting system maps the underlying state of the world into signals reported to the investor. ${ }^{7}$ The mapping rule determines the informational properties of the reported signal, including the likelihood of reporting good versus bad news (i.e., the bias in the disclosure), as well as the investor's residual uncertainty about the project's profitability upon receiving the disclosure (i.e., the informativeness of the disclosure). The manager decides the mapping rule ex ante, that is, prior to receiving any private information about the project's profitability. For my main analysis on the basic model, I follow prior literature in assuming that the manager must truthfully report the signal generated by the system, due, for example, to external auditor verification or legal penalties. I relax this assumption in the extended model of Chapter 4 .

To capture the effect of managerial incentives, I assume the manager has partial ownership of the firm, and, if the project is approved, stands to receive a fraction of the project's payoff as well as a private benefit that does not depend on the project's success. To abstract from the stewardship use of reported information (e.g., Chen et al. (2007), Hemmer and Labro (2008)), I take the manager's ownership share and private benefit as given, and separately analyze the strategies of two types of managers: a mis-aligned manager whose private benefit is so large that he always prefers to invest regardless of the underlying state, and a well-aligned manager who prefers to invest only when the underlying state is good. ${ }^{8}$ Because the investor's

\footnotetext{
7 Viewing disclosure as a signal generated by a reporting system designed ex ante applies naturally to mandatory disclosures, where the existence of the signal, as well as its disclosure to investors, is dictated by the regulatory framework. One can also view firms' voluntary disclosure as generated by a reporting system designed ex ante, where the system follows a set of pre-specified rules to decide whether and how to disclose certain information, as a function of exogenously given and possibly time-varying variables (such as the existing market condition and investors' common prior). This view admits no disclosure as a special case where the ex-ante disclosure rule specifies the release of an uninformative signal when certain conditions are met.

8 I am agnostic about the extent to which firms successfully implement optimal contracts that neutralize the effect of private benefits. To the extent that managers' incentive to over-invest is not
} 
approval decision depends on the information available to her, the manager will choose the mapping rule to influence the investor's information environment, and set the rule as a function of three determinants: his incentive alignment with the investor, the investor's cost of acquiring additional information, and the common prior belief about the underlying state.

I apply the concavification technique developed by Kamenica and Gentzkow (2011) to solve for the manager's optimal reporting system. I find that the misaligned manager will choose an uninformative reporting system, or equivalently, no disclosure, when the investor's prior is optimistic, and will choose an informative but positively biased system when the investor's prior is pessimistic. Furthermore, the optimal system becomes more informative and less liberal when the investor's inspection cost decreases.

The intuition for no disclosure when the investor's prior is optimistic is as follows: Without additional information, the investor's default action is to approve, which is the manager's preferred action. Providing additional information can only lead the investor to choose an action less preferred by the manager. In contrast, when the investor's prior is sufficiently pessimistic so that her default action is to disapprove the project, the mis-aligned manager has nothing to lose by providing informative disclosures. Specifically, the optimal system entails a positive bias in that it is more likely to report good news than bad news. ${ }^{9}$ While this system reduces the investor's posterior (that the true state is good) to zero upon receiving bad news disclosures, it also increases her posterior upon good news disclosures, ${ }^{10}$ which increases the likelihood of approval. In other words, an informative system gives the manager an "option" to benefit from disclosure when it changes the investor's decision in his a prevalent empirical phenomenon, my analysis regarding the reporting strategies of well-aligned managers applies.

${ }^{9} \mathrm{I}$ introduce the formal definition and measurement of bias in Chapter 2.

10 This is because the information system must obey the law of iterated expectations. 
favor. ${ }^{11}$ Furthermore, when the investor can inspect, any positive posterior induced by the good news disclosure needs to be sufficiently informative to reduce the investor's residual uncertainty, so that she would approve without inspection. Since the mis-aligned manager prefers over-investment, he would prefer outright approval (i.e., without inspection) over approval after inspection, because the latter eliminates over-investment. To improve the informativeness of good news disclosures, the system needs to reduce the likelihood of reporting a bad state as good news, i.e., it becomes less liberal and more informative.

For a well-aligned manager, the optimal system is fully revealing (the most informative) when the investor faces a prohibitively high inspection cost, such that firm disclosures are effectively her sole source of information. This is because the well-aligned manager prefers the investor to make the right decision. Therefore, it is in his best interest to disclose all relevant information. However, when the investor's inspection cost is low, the optimal reporting system is no longer fully revealing; instead, it becomes counter-cyclical: It is positively biased when the investor's prior is pessimistic and negatively biased when the prior is optimistic. The intuition is that, unlike the mis-aligned manager who designs the system to discourage investor inspection, the well-aligned manager designs the system to motivate investor inspection, since inspection reveals the true state and ensures the right decision will be made. Investor inspection is particularly valuable when the opportunity cost of the investor's default action (in the absence of inspection) is high. Thus, when the investor's prior is high, the default action is to approve without inspection, which is costly when the true state is bad. A conservative system is more likely to provide "warnings" (in that it is more likely to report low signals), casting doubt on the default action, and therefore, motivating the investor to inspect. On the other hand, when the investor's

11 While a negatively biased system also buys the manager this "option", it is, compared to the positively biased system, less likely to be "in the money" and is therefore not his optimal choice. 
prior is low, the investor's default action is to disapprove, which is costly when the true state is good. A positively biased system is more likely to disclose a high signal, also casting doubt on the default action and inducing inspection.

The basic model assumes that the manager must truthfully disclose the signal generated by the system. This corresponds to the case where no information asymmetry exists between the manager and the investor at the time of the disclosure. In the extended model, I relax this assumption and allow the manager to alter the disclosure after privately observing the original signal generated by the system, albeit at a cost. This corresponds to the case where the manager has more, but still incomplete information about future profitability than does an outside investor. I find that the qualitative results from the no-manipulation case carry through. In fact, the mis-aligned manager is strictly better off if he can credibly commit not to manipulate ex post. The reason is that the manager can replicate every distribution of post-manipulation posteriors under the no-manipulation case, and hence receives the same expected gross payoff, but without the cost of manipulation. As such, allowing manipulation does not change the well-aligned manager's optimal system ex ante as he will never manipulate ex post. However, it may increase the informativeness of a mis-aligned manager's ex-ante optimal system (compared to cases when manipulation is not possible). This is because investors are less responsive to reported signals if they suspect manipulation, forcing the manager to counteract the suspicion by increasing the informativeness of the system. In addition, I find that a larger scope of manipulation can actually improve investment efficiency in firms with mis-aligned managers and low investor inspection costs, because it makes investors more cautious and more likely to acquire additional information. 


\subsection{Related literature}

This paper contributes to the literature on Bayesian persuasion (Kamenica and Gentzkow (2011), Gentzkow and Kamenica (2014), Michaeli (2014), Bertomeu and Cheynel (2015), Friedman et al. (2015)). Kamenica and Gentzkow (2011) provide a solution algorithm for a set of communication games in which the sender influences a rational Bayesian receiver's information environment, with the goal of inducing the receiver to change her action. This resembles my setting in which a manager (the sender) designs a reporting system in order to induce the investor (the receiver) to take the action desired by the manager. While the sender can perfectly control the receiver's information environment in Kamenica and Gentzkow (2011), my model allows the investor to acquire additional information.

I relax the commitment assumption in the extended model, described in Chapter 4, and study to what extent the results generalize to a setting where the manager privately observes the output of the reporting system and can manipulate at a cost ex post. The extended model furthers our understanding of how managerial manipulation affects the properties of the accounting system (e.g., Arya et al. (1998), Laux (2014), Beyer et al. (2014), Gao (2013)). For example, Gao (2013) shows that, to safeguard against ex post managerial manipulation, the socially optimal ex ante accounting rule will require more verification for transaction characteristics favorable to managers. The extended model studies the optimal ex ante reporting system from the managers' perspective when they anticipate the possibility of future opportunistic manipulation.

I also extend the literature on public information disclosures and investors' private information acquisition (e.g., Demski and Feltham (1994), Diamond (1985), McNichols and Trueman (1994), Kim and Verrecchia (1997), Gao and Liang (2013)) to a setting in which disclosures affect investment efficiency. This paper also con- 
tributes to the literature on the interaction between firm investment and disclosure decisions, which highlights the real effects of accounting disclosures (Kanodia et al. (2004, 2005), Sapra (2002), Kumar et al. (2012), Beyer and Guttman (2012)). Beyer and Guttman (2012) study a setting in which a manager chooses jointly an investment, whether to disclose it and whether to raise capital. My paper differs in that I study the disclosure of forward-looking information about the firm's investment opportunities, and I model the manager's disclosure strategy as designing a reporting system ex ante.

There is a large literature on the properties of the optimal reporting system in specific settings, such as debt contracting (e.g., Goex and Wagenhofer (2009), Gigler et al. (2009), Jiang (2012), Caskey and Hughes (2011)) and performance measurement (e.g., Gigler and Hemmer (2001), Chen et al. (2007)). For example, Goex and Wagenhofer (2009) study a setting in which the firm reports the value of collateral to a lender to obtain financing for a risky project, and the manager is subject to moral hazard. They find that the optimal accounting system reports impairments only when the collateral's value falls below a threshold level. Gigler et al. (2009) find that the optimal accounting system is conditionally liberal in a debt contracting setting, because the cost of falsely liquidating a good project is larger than the cost of wrongly continuing a bad project. Chen et al. (2007) find that the optimal accounting system is conservative when accounting serves both a valuation and a performance measurement role. Jiang and Yang (2015) study the properties of optimal accounting standards in a setting where disclosure can reveal a finite amount of information and a seller can signal her private information by retaining assets, and find that the optimal system features revealing an infimum. My paper contributes to this stream of literature by analyzing a firm's optimal reporting system when disclosures affect the investor's information acquisition and intervention decisions.

The paper is organized as follows. Chapter 2 introduces the basic model with- 
out manipulation. Chapter 3 derives the optimal reporting systems for four cases: mis-aligned versus well-aligned managers, and high versus low investor inspection costs. Within each case, the common prior belief is allowed to vary. Chapter 4 studies the extended model with private information to the manager and possible ex-post manipulation. Chapter 5 concludes. Appendix A contains detailed proofs, and Appendix B presents a tabular summary of the results. 


\section{The Basic Model}

The model spans five dates $t \in\{0,1,2,3,4\}$ and involves two risk-neutral players, the manager and the investor.

\subsection{The firm and the manager}

At $t=0$, the firm is endowed with an investment opportunity that requires an initial fixed investment $I$ and needs the representative investor's approval to proceed. The project produces a random cash flow, which is $R>0$ if the project succeeds and 0 if it fails. The project succeeds with probability $\theta_{\omega}$ and fails with probability $1-\theta_{\omega}$, where $\omega \in\{g, b\}$ refers to the underlying state of the project, which can be either good $(g)$ or bad $(b)$. Both the manager and the investor hold the common prior that the project is in the good state with probability $\mu_{0} \in(0,1)$. Without loss of generality, I assume the NPV of the project in the good (bad) state is positive (negative):

$$
\begin{aligned}
& N_{g}=\theta_{g} R-I>0 \\
& N_{b}=\theta_{b} R-I<0
\end{aligned}
$$


The manager owns $s$ percent of the firm's equity and the investor holds the remaining $1-s$ percent. If the project is approved, the resulting cash flow is shared between the manager and the investor according to their equity shares, and the manager also receives a non-contractible, fixed private benefit $B$ from the project. The manager aims to maximize his expected payoff, including the private benefit. I assume the manager's private benefit is too small to compensate for the total loss from investing in a bad project, i.e., $B+N_{b}<0$.

While investing in a good project $(\omega=g)$ benefits both the manager and the investor, investing in a bad project hurts the investor, but may benefit the manager. Specifically, if $B+s N_{b}>0$, the manager's private benefit exceeds his share of the loss from the bad project, and his incentive is mis-aligned with the investor's. If $B+s N_{b}<0$, the manager's private benefit does not compensate for his share of the loss, and his incentive is well-aligned with the investor's. Both the equity share $s$ and the private benefit $B$ are common knowledge, so the investor knows whether the incentives are well-aligned. ${ }^{1}$ I show that incentive-alignment is critical in shaping the firm's reporting system, and discuss the mis-aligned and well-aligned manager's cases separately.

\subsection{Disclosure strategy as the design of a reporting system}

The manager chooses a reporting system ex ante, i.e., before he learns any private information. More specifically, at $t=1$, the manager determines the disclosure strategy by designing a reporting system which produces a high or low signal $\pi \in$ $\{h, l\}$ about the firm's true state $\omega \in\{g, b\}$ at $t=2$, with

$$
\operatorname{Pr}(h \mid g)=\lambda_{g} \geqslant \lambda_{b}=\operatorname{Pr}(h \mid b)
$$

1 The assumption that the incentive-alignment is known to the investor is not critical. Under the alternative assumption that the manager privately knows his incentive, the results remain the same. The well-aligned and the mis-aligned managers will choose different reporting systems, which reveals their type, and they will not have an incentive to mimic the other type. 


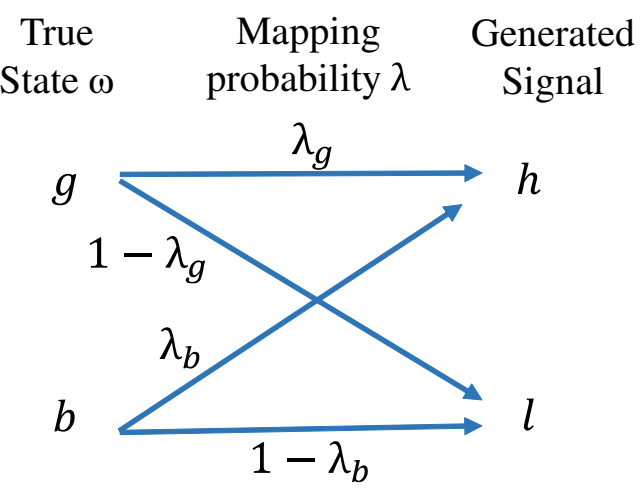

Figure 2.1: The Reporting System in the Basic Model

Figure 2.1 illustrates how the reporting system maps each underlying state into a signal. Given that the investor has two choices, approve or reject the project, the reporting system is, without loss of generality, limited to produce a binary signal. ${ }^{2}$ In the basic model, the manager must truthfully disclose the signal generated by the reporting system. ${ }^{3}$

The properties of the reporting system, $\lambda_{g}$ and $\lambda_{b}$, become common knowledge after they have been determined by the manager. ${ }^{4}$ An important feature of this binary structure is that the manager chooses both the informativeness and the bias of the reporting system. I define these two properties as follows:

Neutrality and bias. The reporting system is neutral if the probability of a good project generating a high signal is equal to the probability of a bad project generating a low signal, i.e., $\lambda_{g}=1-\lambda_{b}$. A positively biased (liberal) reporting system has a comparatively higher probability of a good project generating a high signal, relative

2 The payoff from more detailed reporting systems can be replicated by a simple binary system. To the extent more detailed reporting systems are more costly, a binary system is strictly preferred.

${ }^{3}$ In Chapter 4, I relax this assumption and allow the manager to privately observe the original signal and choose whether to manipulate that signal.

${ }^{4}$ In practice, the properties of the reported information can be disclosed in critical accounting policies or footnotes, or investors can learn over time the properties of reported information through comparing it with realized outcomes. 
to the probability of a bad project generating a low signal, i.e., $\lambda_{g}>1-\lambda_{b}$. A negatively biased (conservative) reporting system generates a high signal with a comparatively lower probability, i.e., $\lambda_{g}<1-\lambda_{b}$.

To compare the relative levels of bias between two reporting systems, I follow Gigler et al. (2009). ${ }^{5}$ A reporting system with $\pi\left(\lambda_{g}, \lambda_{b}\right)$ is more conservative (i.e., less liberal) than an alternative system $\pi^{\prime}\left(\lambda_{g}^{\prime}, \lambda_{b}^{\prime}\right)$, if $\operatorname{Pr}(g \mid \pi=h) \geqslant \operatorname{Pr}\left(g \mid \pi^{\prime}=h\right)$ and $\operatorname{Pr}(g \mid \pi=l) \geqslant \operatorname{Pr}\left(g \mid \pi^{\prime}=l\right)$, with at least one inequality holding strictly. As conservatism increases, the reporting system requires a higher threshold for reporting good news relative to bad news, meaning it is less likely the system will produce a high signal $h$. To the investor, a high signal from a conservative system is more convincing evidence of a good project, while a low signal is less indicative of a bad project, since more good projects are pooled to receive low signals. ${ }^{6}$

Informativeness. The informativeness of a system is measured as the expected reduction in uncertainty relative to a fixed reference belief $\mu_{r}$. I use the entropy measure for uncertainty. If the investor's belief that the state is good is $\mu$, the entropy is $H(\mu)=-\mu \ln \mu-(1-\mu) \ln (1-\mu)$. The informativeness of the system is measured as $L(\pi)=H\left(\mu_{r}\right)-\mathbb{E}_{\pi \mid \mu_{r}}\left(H\left(\mu_{\pi}\right)\right)$, where $\mu_{\pi}$ is the investor's posterior belief upon receiving the disclosure. In the limit, the system is defined as uninformative if the prior remains unchanged after the disclosure.

In practice, a reporting system is costly, and more so when it is more informative (Sims (2006) and Gentzkow and Kamenica (2014)). To capture this observation, I

5 The condition of $\operatorname{Pr}(g \mid h, \pi) \geqslant \operatorname{Pr}\left(g \mid h, \pi^{\prime}\right)$ and $\operatorname{Pr}(g \mid l, \pi) \geqslant \operatorname{Pr}\left(g \mid l, \pi^{\prime}\right)$ can be rewritten as $\frac{\operatorname{Pr}(h \mid g, \pi)}{\operatorname{Pr}(h \mid b, \pi)} \geqslant \frac{\operatorname{Pr}\left(h \mid g, \pi^{\prime}\right)}{\operatorname{Pr}\left(h \mid b, \pi^{\prime}\right)}$ and $\frac{\operatorname{Pr}(l \mid g, \pi)}{\operatorname{Pr}(l \mid b, \pi)} \geqslant \frac{\operatorname{Pr}\left(l \mid g, \pi^{\prime}\right)}{\operatorname{Pr}\left(l \mid b, \pi^{\prime}\right)}$, which is Gigler et al.'s (2009) Condition (A3).

6 The Gigler et al. (2009) definition may not always rank two systems in terms of the degree of conservatism. In other words, there are pairs of systems that cannot be distinguished under this definition. 
model the cost of the reporting system as proportional to its informativeness:

$$
c(\pi)=k L(\pi)=k\left(H\left(\mu_{r}\right)-\mathbb{E}_{\pi \mid \mu_{r}}\left(H\left(\mu_{\pi}\right)\right)\right)
$$

where $k$ is the cost for a one-unit reduction in entropy. ${ }^{7}$

I derive the optimal reporting system for the general case with $k>0$, and characterize its bias and informativeness. However, there is generally no closed-form solution in the $k>0$ case; to keep tractability and to highlight the intuition, in the basic model, I assume that the per-unit cost of the system is positive but approaches zero $\left(k \rightarrow 0^{+}\right){ }^{8}$ The optimal system is obtained by taking the limit of the optimal system in the $k>0$ case, and it is unique. The qualitative results in the $k \rightarrow 0^{+}$ case generalize to the case where $k>0$.

\subsection{The investor}

The investor is Bayesian and interprets the firm's disclosure rationally. After receiving the disclosure at $t=3$, the investor derives her posterior belief that the firm is in the good state $(\mu)$, and chooses whether to inspect, i.e., to collect additional information about the firm. If she chooses to inspect, she will incur an inspection cost $m$ and observe the true state of the firm $\omega \in\{g, b\}$ perfectly. She then decides whether to invest in the firm on the basis of her information set. The investor aims to maximize her expected payoff $u(\mu, a)$, where $a$ is her action. At $t=4$, the payoffs are realized. Figure 2.2 summarizes the sequence of events in the model.

The project proceeds only if the investor approves, in which case the manager obtains the private benefit $B$. Since my main focus is to examine how investors'

7 Following Gentzkow and Kamenica (2014), I measure uncertainty reduction against a fixed reference belief $\mu_{r}$, rather than the prior $\mu_{0}$, to ensure that the cost of reporting system does not vary with prior beliefs.

8 The cost function does not have to follow an entropy specification; other cost functions would work as well. The key assumption is that, all else equal, the manager prefers a less informative system to a more informative and hence hence more costly one. 


\begin{tabular}{lll}
\multicolumn{1}{c}{$t=0$} & $t=2$
\end{tabular}

Figure 2.2: The Timeline in the Basic Model

information acquisition affects firms' disclosures, I model investors as a single representative investor, and abstract from the approval process. In practice, the approval can be explicit, for example, in the form of shareholder voting on major strategic initiatives such as acquisitions. It can also be implicit in the form of investors pricing firms' securities, or providing capital to firms seeking external financing. The key feature in these approval mechanisms is that disclosures affect investors' decisions, and in turn, affect managers' payoff. In my model, investors have the option to acquire costly, private information, in addition to firms' disclosures, and their information acquisition is not contractible. To avoid trivializing the problem, I also assume the manager cannot internalize the investor's inspection cost, which creates a friction insofar as the manager may free-ride on the investor's information acquisition. 


\section{3 \\ Optimal Reporting Systems}

In this chapter, I derive the optimal reporting system in the basic model using backward induction. Specifically, I first derive the investor's optimal response to the disclosure at $t=2$. I then analyze the manager's problem at $t=1$, when his anticipation of the (subsequent) investor reaction will shape the ex-ante optimal reporting system.

\subsection{Investor's reaction to disclosure}

At $t=3$, i.e., after receiving the firm's disclosure, the investor updates her belief about the firm's state, and makes two decisions: whether to inspect, and whether to invest. ${ }^{1}$ The investor's set of possible actions $(\mathbb{A})$ includes three choices: to reject the investment outright $(N)$, to approve the investment outright $(A)$, or to inspect and invest only if inspection reveals that the project is good $(I)$. Let the investor's expected payoff be denoted by $u(\mu, a)$, where $\mu$ is the investor's posterior belief about the state being good, and $a \in \mathbb{A}=\{A, I, R\}$ is the investor's action. The expected

\footnotetext{
${ }^{1}$ This subgame, in this basic characterization, is similar to Povel et al. (2007).
} 
payoffs from each of the three choices are listed below:

$$
u(\mu, a)=\left\{\begin{array}{l}
u(\mu, a=A)=(1-s)\left(\mu N_{g}+(1-\mu) N_{b}\right) \\
u(\mu, a=I)=(1-s) \mu N_{g}-m \\
u(\mu, a=R)=0
\end{array}\right.
$$

where the payoff from outright rejection is standardized to be zero, i.e., $u(\mu, a=R)=$ 0. For completeness, and without loss of generality, I assume that when indifferent between two actions, the investor will take the action the manager prefers.

Obviously, the investor's optimal action depends on her posterior belief about the state being good $(\mu)$, as summarized by the following Lemma.

Lemma 1. Define $\bar{m}=\frac{(1-s) N_{g}\left|N_{b}\right|}{\left|N_{b}\right|+N_{g}}, \bar{\mu}=\frac{\left|N_{b}\right|}{\left|N_{b}\right|+N_{g}}, \bar{\mu}_{1}=\frac{m}{(1-s) N_{g}}$ and $\bar{\mu}_{2}=1-\frac{m}{(1-s)\left|N_{b}\right|}$.

1. When $m>\bar{m}$, the investor will approve outright if $\mu \geqslant \bar{\mu}$ and will reject outright if $\mu<\bar{\mu}$.

2. When $m<\bar{m}$, the investor will inspect and invest only in good projects if $\bar{\mu}_{1}<\mu<\bar{\mu}_{2}$; the investor will reject outright if $\mu<\bar{\mu}_{1}$, and invest without inspection if $\mu>\bar{\mu}_{2}$.

Lemma 1 can be proved by noting that $\left\{\bar{\mu}, \bar{\mu}_{1}, \bar{\mu}_{2}\right\}$ are the three threshold levels of the investor's posterior belief at which the investor is indifferent between two of her potential choices. Specifically, at $\bar{\mu}$ the investor is indifferent between directly approving and rejecting the investment, i.e., $u(\bar{\mu}, a=A)=0$. Since $u(\mu, a=A)$ is increasing in $\mu$, the investor strictly prefers investing without inspection to rejecting when $\mu \geqslant \bar{\mu}$. Likewise, at $\bar{\mu}_{1}$ the investor is indifferent between inspecting and rejecting; and at $\bar{\mu}_{2}$ the investor is indifferent between investing outright and inspecting. When $\mu>\bar{\mu}_{1}$, the investor strictly prefers inspecting over rejecting; when $\mu<\bar{\mu}_{2}$, the investor strictly prefers inspecting over investing outright. 


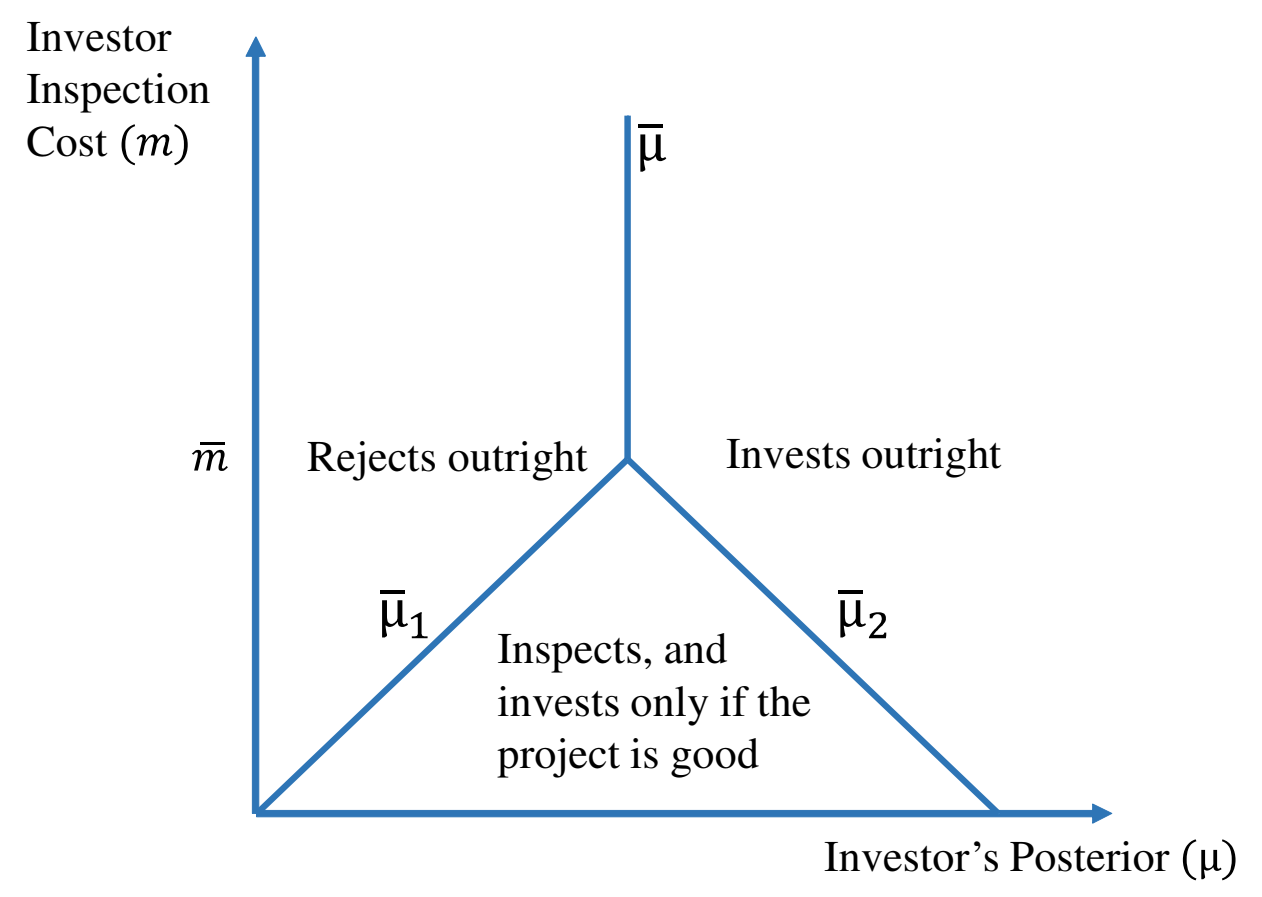

Figure 3.1: The Investor's Optimal Action

Overall, inspecting is optimal only when $\bar{\mu}_{1}<\mu<\bar{\mu}_{2}$, which is the case only when $m<\bar{m}$, where $\bar{m}$ is the threshold inspection cost that equates $\bar{\mu}_{1}=\bar{\mu}_{2}$. That is, $\bar{m}$ represents the threshold inspection cost above which the investor will never inspect, in which case the investor either approves the investment outright if her posterior is sufficiently high (i.e., $\mu \geqslant \bar{\mu}$ ), or rejects outright. With $m<\bar{m}$, the investor optimally inspects if her posterior is in the intermediate range $\left(\bar{\mu}_{1}<\mu<\bar{\mu}_{2}\right)$, rejects outright if her posterior falls below $\left(\mu<\bar{\mu}_{1}\right)$, and approves outright if her posterior is sufficiently high $\left(\mu>\bar{\mu}_{2}\right)$. This result is represented in Figure 3.1.

The intuition is as follows. Inspection benefits the investor by allowing her to avoid wrong decisions. When $\mu$ is small, the investor's default action is to reject the project. Inspection therefore creates value by saving good projects from rejection, and this benefit is higher when the true underlying state is more likely to be good (i.e., when $\mu$ lies closer to the upper end of the rejection region). When $\mu$ is close to 1 , the 
investor's default action is to invest. Inspection therefore creates value by rejecting bad projects, and this benefit is larger when the true state is more likely to be bad (i.e., when $\mu$ lies closer to the lower end of the approval zone). Combined, the benefit of inspection is largest with $\mu$ in the intermediate range, where uncertainty is greatest. A decrease in the inspection cost $m$ leads to an expansion of the intermediate range, corresponding to an increase in the likelihood of inspection.

\subsection{The manager's problem of designing a reporting system}

At $t=3$, the manager's expected payoff $v(\mu, a)$ is determined by the investor's posterior $(\mu)$ and action $(a \in\{A, I, R\})$. His expected payoffs under the investor's actions are as follows:

$$
v(\mu, a)=\left\{\begin{array}{l}
v(\mu, a=A)=B+s\left(\mu N_{g}+(1-\mu) N_{b}\right) \\
v(\mu, a=I)=\mu\left(B+s N_{g}\right) \\
v(\mu, a=R)=0
\end{array}\right.
$$

For a given $m$, the investor's action $a$ is determined solely by her posterior $\mu$, and the manager's payoff is determined by $v(\mu, a(\mu))$, which I denote as $\hat{v}(\mu)$.

At $t=1$, the commonly known prior belief that the state is good is $\mu_{0}$, and there is no information asymmetry between the manager and the investor. The manager designs the reporting system by choosing the mapping probabilities $\lambda_{g}$ and $\lambda_{b}$. He knows the investor is Bayesian and will update her posterior based on the disclosure. Denote the investor's posterior about the state being good upon seeing a high $(h)$ or a low $(l)$ signal as $\mu_{h}$ and $\mu_{l}$, respectively, with

$$
\begin{aligned}
& \mu_{h}=\operatorname{Pr}(g \mid \pi=h)=\frac{\mu_{0} \lambda_{g}}{\mu_{0} \lambda_{g}+\left(1-\mu_{0}\right) \lambda_{b}} \\
& \mu_{l}=\operatorname{Pr}(g \mid \pi=l)=\frac{\mu_{0}\left(1-\lambda_{g}\right)}{\mu_{0}\left(1-\lambda_{g}\right)+\left(1-\mu_{0}\right)\left(1-\lambda_{b}\right)}
\end{aligned}
$$


Conditional on the prior $\mu_{0}$, the manager's choice of $\lambda_{g}$ and $\lambda_{b}$ fully determines $\mu_{h}$ and $\mu_{l}$. Without loss of generality, the manager's problem is to design a reporting system that generates a distribution of posteriors $\left(\mu_{h}, \mu_{l}\right)$, which, in turn, maximizes his gross expected payoff $\mathbb{E}(\hat{v}(\mu))$ less the cost of the reporting system $c(\pi)$. The problem can be rewritten as to maximize $\mathbb{E}\left(\hat{v}_{c}(\mu)\right)$, where $\hat{v}_{c}(\mu)$ is a function of the investor's posterior belief:

$$
\begin{aligned}
V\left(\mu_{0}\right) & =\max _{\mu_{h}, \mu_{l}} \mathbb{E}\left(\hat{v}_{c}(\mu)\right) \\
\text { s.t. } \mu_{0} & =\operatorname{Pr}\left(\mu_{h}\right) \mu_{h}+\left(1-\operatorname{Pr}\left(\mu_{h}\right)\right) \mu_{l} \\
a(\mu) & \in \arg \max _{a \in\{N, I, M\}} u(\mu, a) \\
\hat{v}_{c}(\mu) & =v(\mu, a(\mu))-k\left(H\left(\mu_{r}\right)-H\left(\mu_{\pi}\left(\mu, \mu_{r}\right)\right)\right)
\end{aligned}
$$

Constraint (3.4a) states that Bayesian updating requires the expectation of posteriors to equal the prior. Constraint (3.4b) states that the investor will choose the action that maximizes her payoff, conditional on her posterior. Finally, Constraint (3.4c) writes the manager's conditional expected payoff as of $t=3$ as a function of the investor's posterior. This enables me to rearrange the manager's problem as choosing the distribution of posteriors and facilitate graphic representation. When the cost of the reporting system approaches zero $\left(k \rightarrow 0^{+}\right)$, Constraint $(3.4 \mathrm{c})$ approaches the gross payoff: ${ }^{2}$

$$
\hat{v}_{c}(\mu) \rightarrow \hat{v}(\mu)=v(\mu, a(\mu))
$$

In the following discussion of optimal reporting systems, I distinguish four cases along the two dichotomous dimensions of incentive alignment and investor inspection cost. Section 3.3 (3.4) contains the analysis of a mis-aligned (well-aligned) manager;

\footnotetext{
${ }^{2}$ Under a reporting system $\pi$, if the investor with prior $\mu_{0}$ has posterior $\mu$, then the posterior of the investor with the prior $\mu_{r}$ (the fixed reference belief) can be determined as a function of $\mu$ and $\mu_{r}$, denoted as $\mu_{\pi}\left(\mu, \mu_{r}\right)$.
} 
Sections 3.3.1 and 3.4.1 (3.3.2 and 3.4.2) assume a high (low) cost of investor inspection.

\subsection{Mis-aligned manager's optimal reporting system}

In the basic model, I present results assuming the reporting system's cost is positive and approaches zero to highlight the intuition. These results can be viewed as representing the (realistic) situation where the cost of the reporting system is low compared to the collective inspection costs of all investors. The results hold qualitatively in the more general case of $k>0$.

I first discuss the optimal reporting system of a mis-aligned manager, whose private benefits are sufficiently large that he always prefers investment. Proposition 1 characterizes his optimal reporting system, which depends on whether the investor inspection cost $m$ is above or below the threshold level $\bar{m}$.

Proposition 1. The mis-aligned manager's optimal reporting system is as follows:

1. If $m \geqslant \bar{m}$ :

(a) If $\mu_{0} \geqslant \bar{\mu}$ : The optimal system has $\lambda_{g}=\lambda_{b}$, and is therefore uninformative, yielding the posteriors $\mu_{h}=\mu_{l}=\mu_{0}$.

(b) If $\mu_{0}<\bar{\mu}$ : The optimal system has $\lambda_{g}=1$ and $\lambda_{b}=\frac{\mu_{0}}{1-\mu_{0}} \frac{1-\bar{\mu}}{\bar{\mu}}$, and is therefore positively biased, yielding the posteriors $\mu_{l}=0$ and $\mu_{h}=\bar{\mu}$.

2. If $m<\bar{m}$ :

(a) If $\mu_{0} \geqslant \bar{\mu}_{2}$ : The optimal system has $\lambda_{g}=\lambda_{b}$, and is therefore uninformative, yielding the posteriors $\mu_{h}=\mu_{l}=\mu_{0}$.

(b) If $\mu_{0}<\bar{\mu}_{2}$ : The optimal system has $\lambda_{g}=1$ and $\lambda_{b}=\frac{\mu_{0}}{1-\mu_{0}} \frac{1-\bar{\mu}_{2}}{\bar{\mu}_{2}}$, and is therefore positively biased, yielding the posteriors $\mu_{l}=0$ and $\mu_{h}=\bar{\mu}_{2}$. 
Proof. All proofs are in Appendix A.

\subsubsection{Mis-aligned manager and high investor inspection costs}

Proposition 1, Part 1 characterizes the mis-aligned manager's optimal reporting system when $m \geqslant \bar{m}$. In this case, the investor's inspection cost is so high that she never inspects, and instead relies solely on the disclosed signal for her investment decision. I start by contrasting the manager's payoff under two extreme reporting systems (an uninformative system and a fully revealing system), and plot them in Panel (a) of Figure 3.2. Specifically, the solid lines plot the manager's expected payoff $\hat{v}\left(\mu_{0}\right)$ under an uninformative system. $\hat{v}\left(\mu_{0}\right)$ is not continuous in $\mu_{0}$ : It equals zero when the market prior is low $\left(\mu_{0}<\bar{\mu}\right)$ because the investor will reject the project. However, when the market prior is high $\left(\mu_{0}>\bar{\mu}\right)$, the investor will approve the project, and the manager's expected payoff is $\hat{v}\left(\mu_{0}\right)=\mu_{0}\left(B+s N_{g}\right)+\left(1-\mu_{0}\right)\left(B+s N_{b}\right)$.

For direct comparison, the dash-dot line depicts the manager's expected payoff when the reporting system truthfully reveals the underlying state (the full information case, FI). In this case, the investor invests only if the state is good, and the manager's expected payoff is linear in the market prior: $v_{F I}\left(\mu_{0}\right)=\mu_{0}\left(B+s N_{g}\right)$.

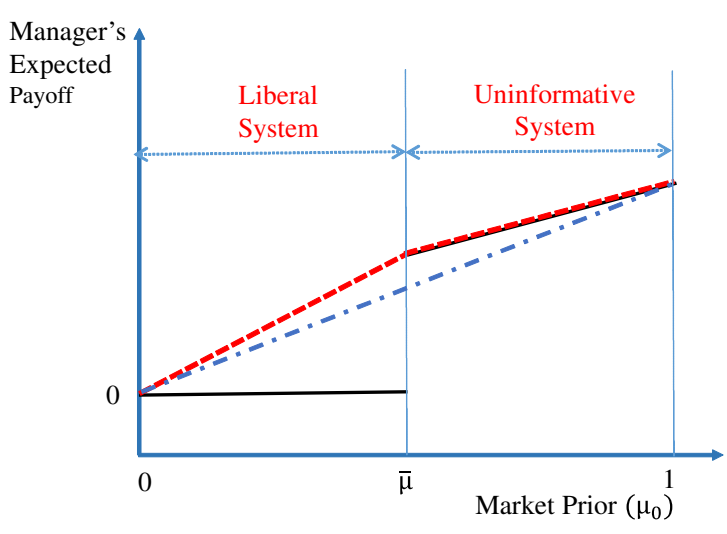

(a) High Inspection Cost

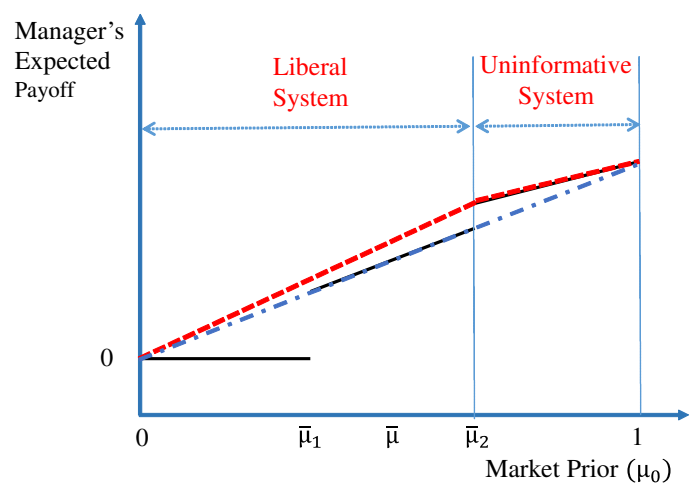

(b) Low Inspection Cost

Figure 3.2: The Mis-aligned Manager's Expected Payoff 
For a mis-aligned manager, $v_{F I}\left(\mu_{0}\right) \leqslant \hat{v}\left(\mu_{0}\right)$ for $\mu_{0}>\bar{\mu}$, and $v_{F I}\left(\mu_{0}\right) \geqslant \hat{v}\left(\mu_{0}\right)$ for $\mu_{0}<\bar{\mu}$. Thus, providing full information hurts (benefits) the mis-aligned manager when the market prior is high (low) and the investor's default action is to approve (reject).

The next question is whether a partially revealing and possibly biased system can improve the manager's expected payoff relative to the two benchmark cases, and under which conditions. I use the concavification technique (Kamenica and Gentzkow (2011)) to derive the optimal system. ${ }^{3}$ In Figure 3(a), the dashed line depicts $V\left(\mu_{0}\right)$, the manager's maximum expected payoff from the optimal reporting system.

The optimal reporting system varies with the market prior. When the market prior is high $\left(\mu_{0} \geqslant \bar{\mu}\right)$, a mis-aligned manager's optimal system is uninformative. The investor cannot update her prior and will default to approve outright. The reason why an informative system is not optimal (for the manager) is as follows. The manager will be strictly worse off if an informative system yields a posterior of $\mu_{l}$ too low to secure investor approval $\left(\mu_{l}<\bar{\mu}\right)$. If the reporting system yields $\bar{\mu} \leqslant \mu_{l}<\mu_{h}$, all projects will be approved and the manager receives the same expected payoff as in the uninformative case. However, given the cost of the reporting system $k$, the manager will prefer the least informative system among a class of systems with the same expected payoff.

When the market prior is low $\left(\mu_{0}<\bar{\mu}\right)$, on the other hand, a mis-aligned manager benefits from an informative system, since the investor's default action is not to invest. Since the investor is rational, the disclosure must be able to change her posterior about whether the project is good. The manager's payoff is strictly higher if the investor is sufficiently convinced to invest; however, his payoff is not reduced below zero if the investor is not sufficiently convinced, since the investor's default

${ }^{3}$ The concavification technique constructs $V\left(\mu_{0}\right)$ as the smallest concave function that is everywhere weakly greater than $\hat{v}\left(\mu_{0}\right)$. 
action is not to invest anyway.

The optimal system has two interesting properties. First, a high signal realization (hereafter, $h$-signal) should be sufficiently convincing, but also not revealing the good state with certainty. The optimal system must induce a posterior of $\mu_{h} \geqslant \bar{\mu}$, because if $\mu_{h}<\bar{\mu}$, the investor will not invest even when she sees a $h$-signal, and the manager gets a payoff of zero. A $h$-signal is not a sure indicator of the good state $\left(\mu_{h}<1\right)$, because the mis-aligned manager privately benefits from investing even in a bad project, and if a $h$-signal is a sure indicator of the good state, no bad project will be invested in. On the margin, the manager can increase his expected payoff by slightly increasing $\lambda_{b}$ from zero. In that case, a $h$-signal still warrants investment, but more (bad) projects are approved. In fact, the optimal system yields the posterior of $\mu_{h}=\bar{\mu}$, whereby the high signal is just sufficiently convincing to ensure approval. This is because, if $\mu_{h}>\bar{\mu}$, the manager can increase his expected payoff by decreasing $\mu_{h}$ while keeping $\mu_{l}$ constant, which increases the probability of obtaining a $h$-signal.

Second, a low signal realization ( $l$-signal) will reveal a bad project with certainty $\left(\mu_{l}=0\right)$. This means that good projects never get a $l$-signal $\left(\lambda_{g}=1\right)$ and are never rejected. The reason is that, if $\mu_{l}>0$, the manager can always increase his expected payoff by decreasing $\mu_{l}$ and keeping $\mu_{h}(\geqslant \bar{\mu})$ constant. This implies higher $\lambda_{g}$ and higher $\lambda_{b}$ : More good projects receive a $h$-signal and are approved, while more bad projects are pooled with good projects in receiving a $h$-signal and are approved as well. For a mis-aligned manager, this strictly increases his expected payoff.

To summarize, a mis-aligned manager's optimal reporting system is positively biased, with more bad projects receiving $h$-signals than good projects receiving $l$ signals. Further, a bad news disclosure (l-signal) is more informative than a good news disclosure ( $h$-signal). Prior empirical research suggests that bad news disclosures are less frequent than good news disclosures, and also more informative insofar as they are followed by larger market reactions (Kothari et al. (2009)). Both the lower 
frequency and the higher informativeness of bad news disclosures are consistent with predictions of my model, when managers' incentives are mis-aligned.

\subsubsection{Mis-aligned manager and low investor inspection costs}

Proposition 1, Part 2 presents the mis-aligned manager's optimal system when the investor's inspection cost is low $(m<\bar{m})$. The investor may realistically acquire additional information if her posterior falls into the intermediate range between posteriors that lead to outright investment and posteriors that lead to rejection. In Panel (b) of Figure 3.2, the dashed line gives $V\left(\mu_{0}\right)$, the manager's maximum expected payoff from the optimal reporting system. The solid line segments depict the mis-aligned manager's expected payoff $\hat{v}\left(\mu_{0}\right)$ when the system is uninformative and the investor's posterior equals her prior. $\hat{v}\left(\mu_{0}\right)$ differs from the case of high inspection costs in that the investor's possible actions now include inspection in an intermediate range of priors.

Graphically, the function $\hat{v}\left(\mu_{0}\right)$ now has two discontinuities, at $\bar{\mu}_{1}$ and $\bar{\mu}_{2}$. When the prior is low $\left(\mu_{0}<\bar{\mu}_{1}\right)$, the investor neither inspects nor invests, and the manager's payoff is zero. When the prior is in the intermediate range $\left(\bar{\mu}_{1} \leqslant \mu<\bar{\mu}_{2}\right)$, without additional disclosure, the investor inspects and invests only in good projects; as a result the manager's payoff is $\mu_{0}\left(B+s N_{g}\right)$, which is linear in the market belief. When the belief is sufficiently high $\left(\mu_{0}>\bar{\mu}_{2}\right)$, the investor invests without inspection and the manager's payoff is $\mu_{0}\left(B+s N_{g}\right)+\left(1-\mu_{0}\right)\left(B+s N_{b}\right)$. The second discontinuity, at $\bar{\mu}_{2}$, is due to a mis-aligned manager's private benefits from investing in bad projects exceeding his share of the loss. To summarize, a fully revealing system will result in a higher (lower) [the same] expected payoff for the mis-aligned manager compared to an uninformative system when market prior is low (high) [intermediate].

Again, a partially revealing system can achieve a higher expected payoff than either a fully revealing or an uninformative system, depending on the market prior. 
When $\mu_{0} \geqslant \bar{\mu}_{2}$, the investor's default action is to invest directly without inspection, the mis-aligned manager's favored action. Thus, the manager's optimal system is uninformative, since an informative system can never improve his expected payoff, but imposes additional costs.

When $\mu_{0}<\bar{\mu}_{1}$, the investor's default action is not to invest, the least preferred action from the manager's point of view. Hence, the manager benefits from an informative system if the system convinces the investor to take other actions with a higher probability. This requires the optimal system to have $\mu_{h} \geqslant \bar{\mu}_{1}$; otherwise, the investor never invests. Furthermore, the optimal system has $\mu_{l}=0$. If not, the manager can always improve his expected payoff by setting $\mu_{l}=0$ while keeping $\mu_{h} \geqslant$ $\bar{\mu}_{1}$ constant, which ensures all good projects get approved $\left(\lambda_{g}=1\right)$, increasing his expected payoff. Finally, the mis-aligned manager achieves the maximum expected payoff by setting $\mu_{h}=\bar{\mu}_{2}$ and keeping $\mu_{l}=0$, since this system enables bad projects to get approved with the highest probability $\left(\lambda_{b}=\frac{\mu_{0}}{1-\mu_{0}} \frac{1-\bar{\mu}_{2}}{\bar{\mu}_{2}}\right)$.

When $\bar{\mu}_{1} \leqslant \mu_{0}<\bar{\mu}_{2}$, the investor's default action is to inspect and invest only in good projects, yielding a positive expected payoff for the manager. However, the manager can improve on this payoff by designing a system that induces approval for all good projects and some bad projects, setting $\mu_{l}=0$ and $\bar{\mu}_{2} \leqslant \mu_{h}<1$. It turns out that the optimal system has $\mu_{h}=\bar{\mu}_{2}$ and $\mu_{l}=0$, which retains the investor's willingness to invest directly in $h$-signal firms, and maximizes the probability of obtaining $h$-signals for both good and bad projects.

Figure 3.3 summarizes how the mis-aligned manager's optimal system changes with the market prior in a numerical example. For $\mu_{0} \in\left(0, \bar{\mu}_{2}\right)$, the manager strictly benefits from the optimal system, while for $\mu_{0} \in\left[\bar{\mu}_{2}, 1\right]$, he prefers an uninformative system. Furthermore, the optimal system becomes less liberal and more informative as the market prior decreases. 


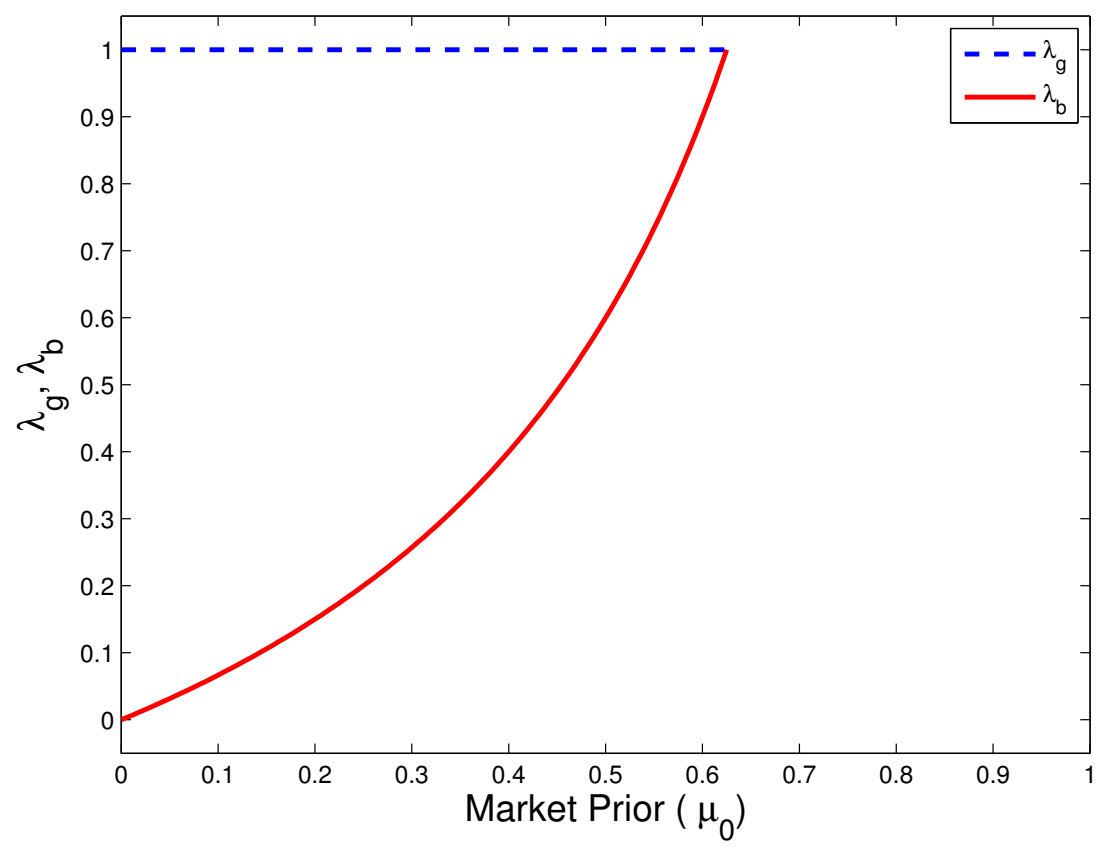

Figure 3.3: The Mis-aligned Manager's Optimal System

Also, $\bar{\mu}_{2}$ is larger than $\bar{\mu}$ when $m<\bar{m}$, i.e., the range of market priors in which the manager prefers an uninformative system, $\left[\bar{\mu}_{2}, 1\right]$, is smaller compared to the high investor inspection costs case. Corollary 1 presents the impact of investor inspection costs on firm disclosures. Denote $\tilde{\mu}=\max \left(\bar{\mu}, \bar{\mu}_{2}\right)$ the threshold posterior belief above which the investor invests without inspection. $\tilde{\mu}$ weakly decreases in $m$.

Corollary 1. For a mis-aligned manager, as $m$ decreases:

1. The range of market priors in which an uninformative system is optimal, [ $\tilde{\mu}, 1]$, shrinks; the range over which an informative system is optimal, $(0, \tilde{\mu})$, expands.

2. When the optimal system is informative, it has $\lambda_{g}=1$ and $\lambda_{b}$ weakly decreases as $m$ decreases; the system becomes more informative and less liberal.

A mis-aligned manager's disclosure convinces the investor to approve the investment without inspection. The optimal system will ensure that good projects generate 
a $h$-signal and are approved $\left(\lambda_{g}=1\right)$, and also maximizes the probability that bad projects are approved. Lower investor inspection costs make inspection more likely and require more convincing evidence of a good project. Thus, the manager has to design a more informative and less liberal system.

Overtime, evolving communication and computation technologies have substantially reduced investor inspection costs. At the same time, empirical studies find that firms expanded their financial disclosures (Francis et al. (2002b)), and accounting has become more conservative (Givoly and Hayn (2000)). My model traces both findings to the decrease in inspection costs as a potential explanation. Relatedly, to the extent that investors' inspection costs vary across countries, my model predicts the degree of reporting conservatism will also vary across countries. Ball et al. (2003) find that, despite having high quality accounting standards, the four East Asian countries in their sample exhibit low timeliness in loss recognition. ${ }^{4}$ My model suggests an alternative explanation: Investors in those countries face prohibitively high costs to acquire additional information, which renders them unable to monitor managers effectively. Rational managers will, in turn, report more liberally.

In addition, investor inspection costs have a direct impact on a mis-aligned manager's investment efficiency, as summarized in Corollary 2.

Corollary 2. A mis-aligned manager over-invests with probability $\min \left\{\frac{\mu_{0}}{\tilde{\mu}}, 1\right\}-\mu_{0}$. As the investor inspection cost $m$ decreases, over-investment weakly decreases.

Prior empirical research finds that accounting conservatism is positively associated with investment efficiency. For example, Francis and Martin (2010) find that firms with more timely incorporation of economic losses into earnings make more

4 Gigler et al. (2009) note that their definition of conservatism is consistent with Basu (1997). Since my definition of conservatism follows Gigler et al. (2009), my paper speaks to the empirical findings using the Basu (1997) measure of conservatism. It is not clear whether other proxies of conservatism are consistent with the Gigler et al. (2009) definition, and my results speak to results based on other proxies only to the extent they are consistent with the Gigler et al. (2009) definition. 
profitable acquisitions, and infer that accounting conservatism complements other governance mechanisms to achieve better investment outcomes. My results suggest an alternative explanation: Lower investor inspection costs lead to both more accounting conservatism and better investment efficiency, and conservatism per se does not directly affect investment efficiency. ${ }^{5}$

\subsection{Well-aligned manager's optimal reporting system}

A well-aligned manager differs from a mis-aligned manager in that he prefers not to invest in a bad project because his private benefit is too small to compensate for his share of the project loss. This difference in incentives leads to a large difference in the optimal reporting systems. Proposition 2 characterizes a well-aligned manager's optimal system when the cost of the reporting system is negligible $\left(k \rightarrow 0^{+}\right)$.

Proposition 2. The well-aligned manager's optimal reporting system is as follows:

1. If $m>\bar{m}$ : The optimal system has $\lambda_{g}=1$ and $\lambda_{b}=0$, and therefore is fully revealing and neutral, yielding the posteriors $\mu_{l}=0$ and $\mu_{h}=1$.

2. If $m<\bar{m}$ :

(a) If $\mu_{0} \geqslant \bar{\mu}_{2}$ : The optimal system has $\lambda_{g}=\frac{\mu_{0}-\bar{\mu}_{2}}{\mu_{0}\left(1-\bar{\mu}_{2}\right)}$ and $\lambda_{b}=0$, and is therefore negatively biased, yielding the posteriors $\mu_{l}=\bar{\mu}_{2}$ and $\mu_{h}=1$.

(b) If $\bar{\mu}_{1} \leqslant \mu_{0}<\bar{\mu}_{2}$ : The optimal system has $\lambda_{g}=\lambda_{b}$, and is therefore uninformative, yielding the posteriors $\mu_{h}=\mu_{l}=\mu_{0}$.

(c) If $\mu_{0}<\bar{\mu}_{1}$ : The optimal system has $\lambda_{g}=1$ and $\lambda_{b}=\frac{\mu_{0}}{1-\mu_{0}} \frac{1-\bar{\mu}_{1}}{\bar{\mu}_{1}}$, and is therefore positively biased, yielding the posteriors $\mu_{l}=0$ and $\mu_{h}=\bar{\mu}_{1}$.

\footnotetext{
${ }^{5}$ In the mis-aligned manager's case, lower investor inspection costs lead to higher investment efficiency, but not higher inspection frequency, because the manager improves the informativeness of the reporting system and prevents investor from inspecting.
} 


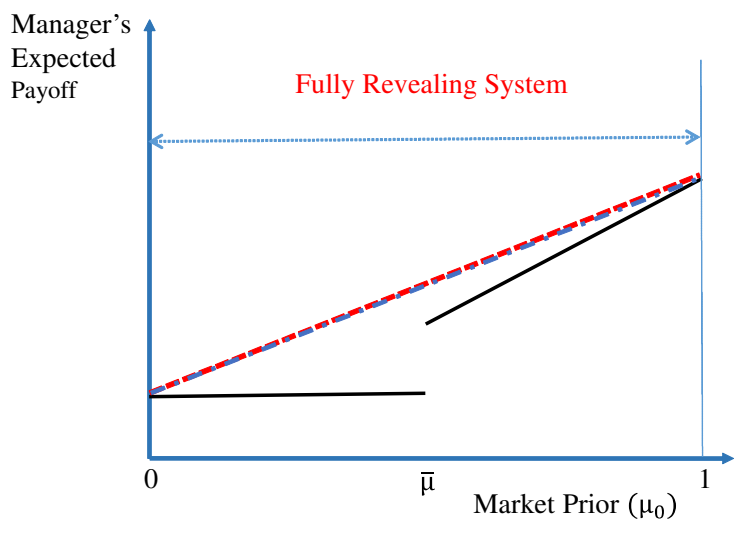

(a) High Inspection Cost

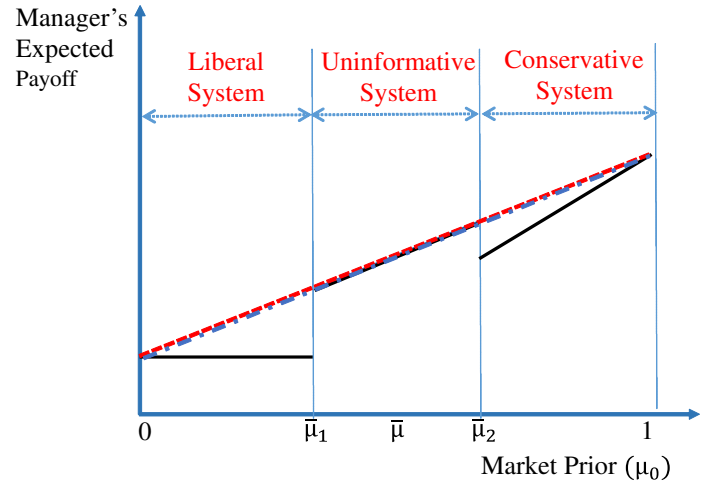

(b) Low Inspection Cost

Figure 3.4: The Well-aligned Manager's Expected Payoff

Figure 3.4 plots the well-aligned manager's expected payoff under different information systems with different investor inspection costs. The solid lines plot his expected payoff $\hat{v}\left(\mu_{0}\right)$ under an uninformative system; the dash-dot lines plot his expected payoff $v_{F I}\left(\mu_{0}\right)$ under a fully-revealing system; and the dashed lines plot his expected payoff $V\left(\mu_{0}\right)$ from the optimal reporting system.

\subsubsection{Well-aligned manager and high investor inspection costs}

Proposition 2, Part 1 characterizes the well-aligned manager's optimal system when the investor's inspection cost is high. In this case, the investor makes decisions relying on the public disclosure only, and the well-aligned manager prefers the investor to invest only if the state is good. As a result, the optimal system is fully revealing.

This result is in sharp contrast with the choice by the mis-aligned manager: The mis-aligned manager prefers an uninformative system when the prior is sufficiently high $\left(\mu_{0} \geqslant \bar{\mu}\right)$, and a partially-revealing and liberal system when the prior is low $\left(\mu_{0}<\bar{\mu}\right)$. The reason is that, while a mis-aligned manager always prefers the investor to invest, a well-aligned manager prefers the investor to make the right decision, and better information facilitates better decision-making. When investor inspection costs 
are prohibitively high, it is optimal for a well-aligned manager to provide the best possible information. If the cost of the system approaches zero, he will choose a fully-revealing system. This is graphically represented in Panel (a) of Figure 3.4, where $V\left(\mu_{0}\right)$ coincides with $v_{F I}\left(\mu_{0}\right)$, the expected payoff in the full-information benchmark.

\subsubsection{Well-aligned manager and low investor inspection costs}

Proposition 2, Part 2 characterizes the well-aligned manager's optimal system when the investor's inspection cost is low, in which case the investor acquires additional information when the disclosure does not sufficiently resolve her uncertainty. The possibility of investor information acquisition changes the well-aligned manager's optimal system from fully revealing to counter-cyclical: It is liberal when the market prior is low, conservative when the market prior is high, and is uninformative when the market prior is intermediate and the investor defaults to inspecting. The intuition is as follows.

When the market prior is high $\left(\mu_{0}>\bar{\mu}_{2}\right)$, the investor approves outright upon receiving a $h$-signal and inspects upon receiving a $l$-signal. Thus, Type-I error by the information system (i.e., reporting a good project as a $l$-signal) is costless, since a good project with a $l$-signal will be revealed by inspection and approved. In contrast, a Type-II error (reporting a bad project with a $h$-signal) is costly, because a $h$-signal leads to approval without inspection, which reduces the payoff of a well-aligned manager because the project is actually bad. Thus it is optimal for the manager to avoid Type-II errors as much as possible, which translates into all bad projects receiving a $l$-signal, even if some good projects also receive a $l$-signal. This renders the optimal system conservative when the market prior is high.

On the other hand, when the market prior is low $\left(\mu_{0}<\bar{\mu}_{1}\right)$, the investor inspects upon receiving a $h$-signal and disapproves without inspection upon receiving 


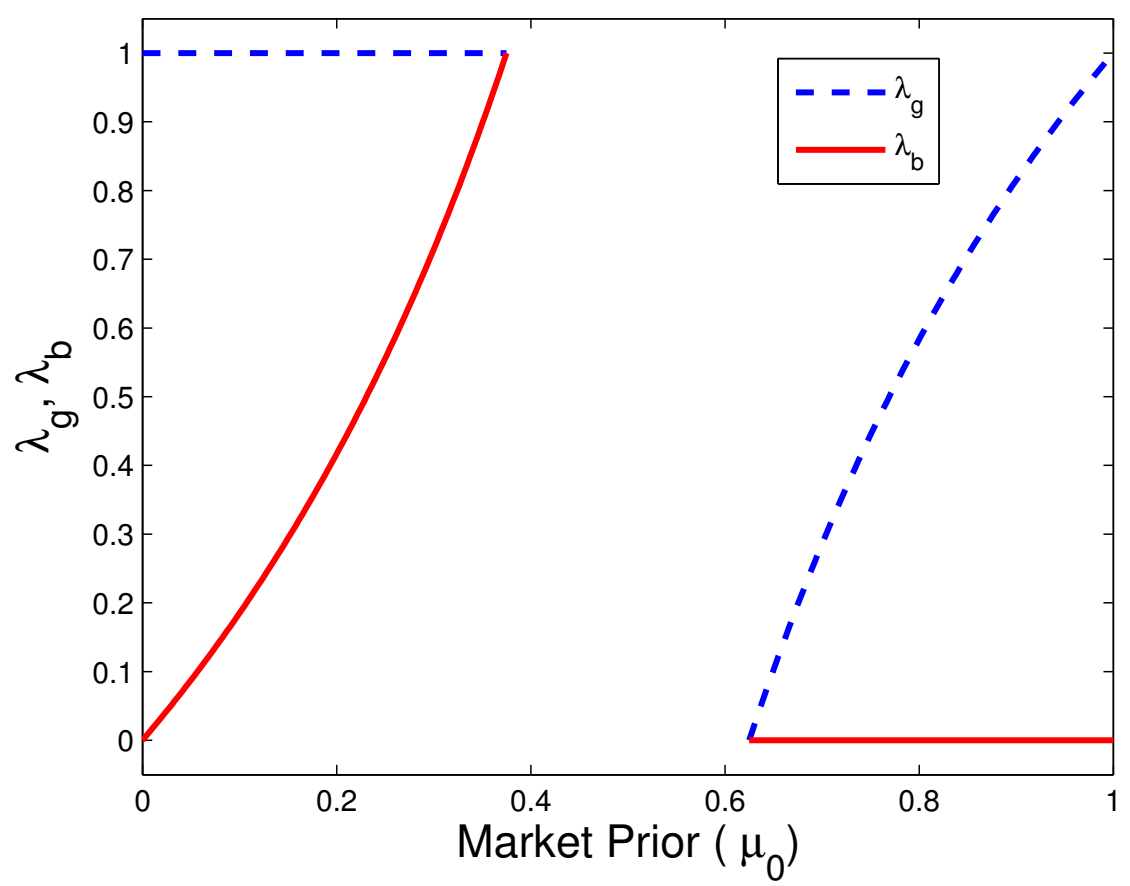

Figure 3.5: The Well-aligned Manager's Optimal System

a $l$-signal. In this case, Type-I errors lead to opportunity losses due to foregone investments with positive NPV. However, a Type-II error is costless, since bad projects with a $h$-signal will be revealed by inspection and rejected. Thus, it is optimal for the manager to avoid Type-I errors as much as possible, which means that all good projects should receive a $h$-signal, even if some bad projects also receive a $h$-signal. This makes the optimal system liberal when market prior is low.

When the market prior is in the intermediate range $\left(\bar{\mu}_{1} \leqslant \mu_{0} \leqslant \bar{\mu}_{2}\right)$, the investor will inspect and invest only in good projects, the well-aligned manager's favored action. Since the reporting system is costly, the manager prefers an uninformative system. Figure 3.5 presents how the well-aligned manager's optimal system changes with the market prior in a numerical example. The system is less biased and more informative when the market prior is less uncertain, i.e., when $\mu_{0}$ approaches 0 or 1 .

The counter-cyclical reporting system resembles the earnings smoothing behavior 
documented in prior literature (e.g., Liu and Ryan (2006)). When the market prior is high and the investor's default action is to invest directly, a conservative system, which requires more income-decreasing accruals, maximizes the probability that bad projects will get inspected and stopped. When the market prior is low and the default action is not to invest, a liberal system, which requires more income-increasing accruals, maximizes the probability that good projects will get inspected and funded.

The counter-cyclical system is also consistent Tucker and Zarowin's (2006) finding of a positive association between earnings smoothness and the ability of stock price to predict future earnings. While Tucker and Zarowin (2006) interpret the finding as managers smooth earnings to improve their predictive ability for future performance, my model suggests that well-aligned managers smooth earnings to reduce their informativeness, and thereby motivate investors' information acquisition. Nonetheless, since the acquired information affects firms' investment decisions and thus future cash flows, and at the same time is also impounded in stock price by investors, stock prices in firms with actively smoothed earnings are expected to be more positively associated with future earnings.

Corollary 3. As $m$ decreases, within $0<m<\bar{m}$, the following results obtain for a well-aligned manager:

1. The range of market priors over which the manager prefers uninformative disclosures, $\mathbb{U}=\left[\bar{\mu}_{1}, \bar{\mu}_{2}\right]$, expands. The range of market prior beliefs over which the manager prefers informative disclosures, $\mathbb{I}=\left(0, \bar{\mu}_{1}\right) \cup\left(\bar{\mu}_{2}, 1\right)$, shrinks.

2. If $\mu_{0} \in\left(0, \bar{\mu}_{1}\right): \lambda_{g}=1$ and $\lambda_{b}$ increases as $m$ decreases. The optimal system becomes less informative and more liberal.

3. If $\mu_{0} \in\left(\bar{\mu}_{2}, 1\right), \lambda_{b}=0$ and $\lambda_{g}$ decreases as $m$ decreases. The optimal system becomes less informative and more conservative. 
One empirical implication of this corollary is that counter-cyclicality is more pronounced when investors face relatively lower inspection costs. Hutton et al. (2012) find that a manager's information advantage resides at the firm level, and an analyst's information advantage resides at the macro-economic level. Analysts provide more accurate earnings forecasts than do managers when earnings correlate more strongly with macro-economic factors such as GDP and energy costs. My model predicts that firms with this feature should benefit more from external information acquisition. As a consequence, these firms will make fewer disclosures, and their disclosures will be more counter-cyclical to the market prior to induce the collection of macro-level information by market participants.

Prior empirical work also provides evidence of income smoothing in firms more exposed to macroeconomic uncertainty. For example, Liu and Ryan (2006) document that commercial banks smooth earnings over business cycles using loan loss provisions, which they interpret as opportunistic behavior. In my model, however, such earnings smoothing may be optimal even if managers and investors are rational. Income-increasing (income-decreasing) accruals in recessions (expansions) induce external information acquisition, which in turn improves investment efficiency.

Perhaps surprisingly, better incentive alignment between the manager and the investor does not necessarily lead to more informative disclosures. Figure 3.6 compares the optimal reporting systems for the mis-aligned and well-aligned managers. For inspection costs $m<\bar{m}$ and market priors $\mu_{0}<\bar{\mu}_{2}$, the disclosure is more informative when the manager is mis-aligned. When $\bar{\mu}_{1}<\mu_{0}<\bar{\mu}_{2}$, the well-aligned manager's optimal system is uninformative, while the mis-aligned manager's is informative. When $\mu_{0}<\bar{\mu}_{1}$, both employ informative systems, but the mis-aligned manager's is more informative and less liberal.

The reason is that a mis-aligned manager uses disclosures to convince the investor to approve the investment, while a well-aligned manager does not prefer uncondi- 


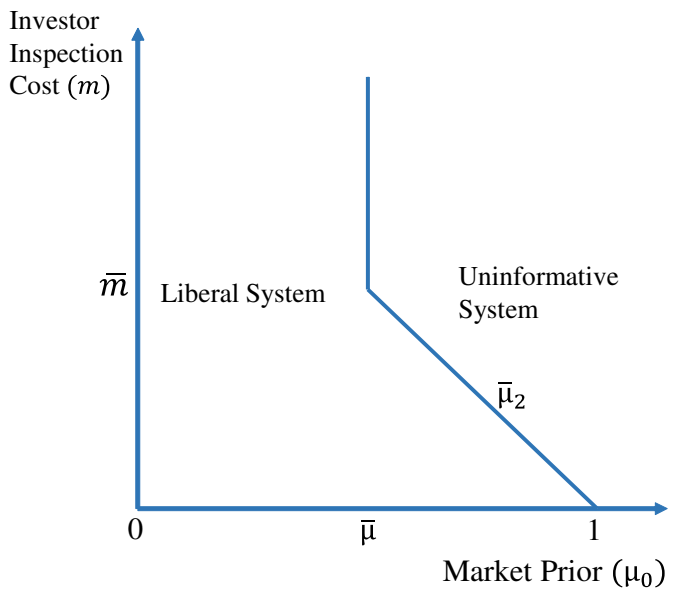

(a) Mis-aligned Manager

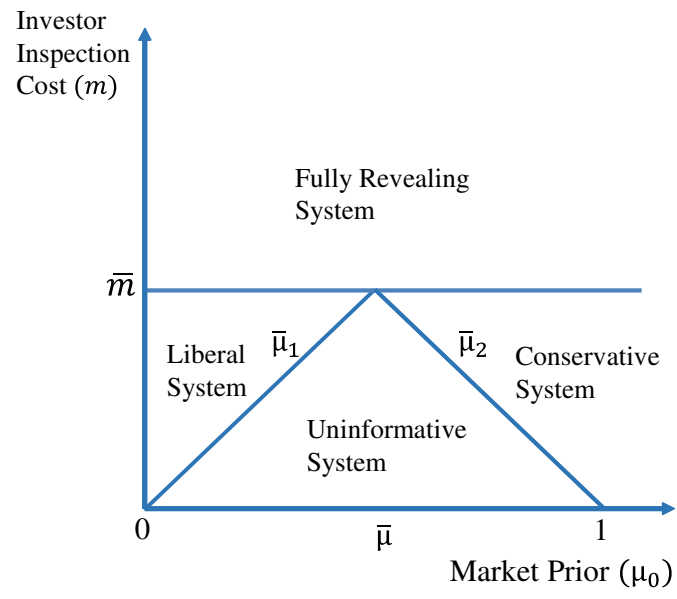

(b) Well-aligned Manager

FiguRE 3.6: Optimal Reporting Systems for Mis-aligned and Well-aligned Managers

tional investing, but rather aims to induce the right investment decision. By disclosing less, he induces information acquisition by the investor, from which he benefits. In its basic form, this result resembles the conclusion in Gao and Liang (2013). The "feedback effect" entails that the firm learns the information collected by investors as aggregated in price. My result highlights that incentive alignment is a key premise for this to happen. Furthermore, in this basic model with $k \rightarrow 0^{+}$, lower investor inspection costs lead to less informative disclosure, but without reducing investment efficiency. In the costly reporting system case $(k>0)$, lower investor inspection costs improve investment efficiency.

\subsection{Further discussions}

In this subsection, I discuss several alternative assumptions, and how they would affect the results in my basic model. 


\subsubsection{Private information about the manager's incentives}

The basic model assumes the manager's equity share $s$ and private benefit $B$ are common knowledge, and thus the investor knows whether the manager's incentive is well-aligned. All results in the basic model hold if the manager, but not the investor, knows his incentive alignment. Under this alternative assumption, the well-aligned manager and the mis-aligned manager pick different optimal systems, and thus reveal their true incentives. Their optimal systems are the same as in the basic model, and neither type has an incentive to mimic the other type.

\subsubsection{Private information when designing the reporting system}

The basic model assumes when designing the reporting system, the manager and the investor have a common prior about project's profitability and there is no information asymmetry. An alternative assumption is that at $t=1$, the manager privately observes an additional piece of information before designing the system. Thus, while the investor's prior is that the state is good with probability $\mu_{0}$, the manager's belief is that the state is good with probability $\mu_{0}^{m}$. The investor does not know $\mu_{0}^{m}$, but know that $\mathbb{E}\left(\mu_{0}^{m}\right)=\mu_{0}$. The manager may choose his reporting system based on his private information. ${ }^{6}$ The results under this alternative assumption depend on the manager's incentive-alignment.

To make the discussion more concrete, in this subsection I assume the manager privately observes an additional binary signal. After observing the private signal, the manager's belief about the state being good can be either $\mu^{\prime}$ or $\mu^{\prime \prime}$, with $\mu^{\prime}>\mu_{0}>\mu^{\prime \prime}$.

For mis-aligned managers, when the investor's prior belief is sufficiently high

\footnotetext{
${ }^{6}$ In the extended model of Chapter 4, the manager privately observes the signal generated by the reporting system, and chooses whether to manipulate it at a cost. This subsection differs from the extended model. Here the manager has private information at the time when choosing the reporting system, while in the extended model the manager does not have private information when designing the system; instead he has private information only after the system in place, and he only has a limited scope of manipulation.
} 
$\left(\mu^{\prime}>\mu^{\prime \prime}>\tilde{\mu}\right)$, both managers will choose the uninformative system. When $\tilde{\mu}>\mu^{\prime \prime}$, the results depend on whether the manager's private information can be truthfully disclosed at a sufficiently low cost $(d)$. If so, the manager with more favorable private information will voluntarily disclose, and the investor can learn managers' private information from their disclosure or non-disclosure.

Lemma 2. Suppose the manager has private information $\mu \in\left(\mu^{\prime}, \mu^{\prime \prime}\right)$ at the time of designing the reporting system. For mis-aligned managers, if the cost to truthfully disclose his private information is sufficiently low, the manager with $\mu^{\prime}$ discloses his private information and the manager with $\mu^{\prime \prime}$ does not. After the disclosure, there is no information asymmetry and the subgame proceeds the same as in the basic model.

Another alternative assumption is that the manager can not truthfully disclose his private information regardless of the cost. In that case, the only equilibrium will be a pooling equilibrium, and the manager chooses the same optimal system as in the basic model. The reason is that the manager with less favorable private information $\left(\mu^{\prime \prime}\right)$ always has an incentive to mimic the manager with more favorable information $\left(\mu^{\prime}\right)$, and as a result the only equilibrium is a pooling one. ${ }^{7}$

Lemma 3. Suppose the manager has private information $\mu \in\left(\mu^{\prime}, \mu^{\prime \prime}\right)$ at the time of designing the reporting system. For mis-aligned managers, if the private information cannot be truthfully disclosed at a sufficiently low cost, his optimal systems are the same as in the basic model.

For well-aligned managers, the optimal system in the basic model remains optimal, regardless of how costly it is to truthfully disclose the private information. This

7 A related paper, Bagnoli and Watts (2005), studies a setting in which the manager has private information about future cash flows and bears a cost if an earnings report falls short of expectations. They find that the manager with more favorable private information signals it by choosing a more conservative system. Signaling does not arise in my setting because the manager with less favorable private information always has an incentive to mimic the other type. 
is because under this system investment is efficient and the well-aligned manager achieves highest expected payoff; thus a pooling equilibrium exists in which both managers with either $\mu^{\prime}$ or $\mu^{\prime \prime}$ have no incentive to reveal their private information.

\subsubsection{Heterogeneous priors}

The basic model assumes the manager and the investor hold common prior beliefs about the project's profitability. Alternatively, the manager and the investor may hold different prior beliefs; for example, the investor's prior about the state being good is $\mu_{0}$, while the manager's prior is $\mu_{0}^{m}$. Under this assumption, the investor and the manager hold different priors, but there is no private information; i.e., they "agree to disagree". From the manager's perspective, the optimal systems remain the same as in the basic model with common prior beliefs $\mu_{0}$. The reason is that the manager designs the system to induce the investor to take the action he prefers; and it is the investor's prior that affects her optimal action, while the manager's prior affects his expected payoff, but does not affect how he ranks the desirability of investor's actions. Thus, the results are robust to heterogeneous prior beliefs.

\subsubsection{Alternative payoff/cost functions of investor inspection}

In the basic model, the investor learns the true state of the project if she inspects. A possible alternative assumption is that, inspection reveals the true state with probability $p \in\left(\frac{1}{2}, 1\right)$, and reveals nothing with probability $1-p$. Under this alternative assumption, the investor's expected payoff from inspection declines, and the range of beliefs $\left(\bar{\mu}_{1}^{\prime}, \bar{\mu}_{2}^{\prime}\right)$ under which her optimal action is to inspect shrinks. ${ }^{8}$

The optimal reporting system for the mis-aligned manager remains qualitatively similar to that in the basic model. The mis-aligned manager will choose an unin-

\footnotetext{
${ }^{8}$ An alternative form of investor inspection cost is that the investor can choose the informativeness of the signal and a more informative signal is more costly. Under this assumption, the model is less tractable, and the main intuition is likely to remain.
} 
formative system if $\mu_{0} \geqslant \max \left(\bar{\mu}, \bar{\mu}_{2}^{\prime}\right)$, and choose a partially informative, positively biased system if $\mu_{0}<\max \left(\bar{\mu}, \bar{\mu}_{2}^{\prime}\right)$. Since $\bar{\mu}_{2}^{\prime}$ declines compared to the basic model, the manager is less likely to choose an informative system. Furthermore, in the range of priors for which the optimal system is partially informative, it becomes more liberal and less informative.

The optimal reporting system for the well-aligned manager is different from that in the basic model. If inspection cannot reveal the true state, the well-aligned manager's expected payoff from a partially revealing system that induces investor inspection is lower. Thus, if the cost of the information system is negligible $(k \rightarrow 0)$, the well-aligned manager's optimal system is fully revealing. ${ }^{9}$

\subsubsection{The manager internalizes the inspection cost}

In the basic model, I assume the manager cannot internalize the investor's inspection cost. As a result, there is a friction even in the well-aligned manager's case in that he free-rides on the investor's information acquisition. This assumption is justifiable in that it is unobservable whether the investor acquires information, and thus information acquisition is not contractible.

The well-aligned manager's incentive is different from the incentive faced by a social planner, because the social planner will take into consideration the investor information acquisition cost. Under the alternative assumption that the manager internalizes the investor inspection cost, the manager will disclose more and rely on investor information acquisition less. Alternatively, if the firm issues equity in an IPO to fund the project, and the IPO price incorporates the future inspection cost, for $k \rightarrow 0^{+}$, the optimal system will be fully revealing. ${ }^{10}$

\footnotetext{
9 This result for the well-aligned manager is due to the assumption that the cost of the reporting system is negligible. For a sufficiently large cost $(k>0)$, a partially revealing system will again be optimal.

${ }^{10}$ If the cost of the reporting system is large $(k>0)$, the fully revealing system will be too costly and a partially revealing, counter-cyclical system may again be optimal.
} 


\subsubsection{Variable investment}

In the basic model, the project has a fixed scale. An alternative assumption is that the investor can choose the amount of investment $I$. For example, the project's NPV is $N_{g} I$ in a good state and $N_{b} I$ in a bad state, and the manager's private benefit is $B I$. This assumption creates an additional benefit of information acquisition, in that the investor can use the information about the underlying state to determine the optimal level of investment. Since investor's inspection happens before she determines the scale of the investment and does not vary with the investment scale, this assumption makes the investor more likely to inspect. As a result, the mis-aligned manager is more likely to choose an informative system to prevent investor information acquisition, while the well-aligned manager is less likely to choose an informative system to induce more investor information acquisition.

\subsubsection{Alternative timeline: investor inspection and manager's choice are simulta- neous}

The basic model assumes the investor decides whether to acquire additional information after observing the firm's disclosure. In persuasion games, the sequence of the events may be critical to the results. What if, in the basic model, the investor inspection happens simultaneously with the design of the reporting system? In this subsection, I assume $m<\bar{\mu}$ so that inspection is optimal for the investor when uncertainty is sufficiently large.

Under this alternative timeline, the equilibrium in the mis-aligned manager's case is as follows. If $\mu_{0} \geqslant \bar{\mu}_{1}$, his optimal system is uninformative, and the investor inspects if $\bar{\mu}_{1}<\mu_{0}<\tilde{\mu}$, and approves outright if $\mu_{0} \geqslant \tilde{\mu}$. If $\mu_{0}<\bar{\mu}_{1}$, his optimal system has $\mu_{h}=\bar{\mu}$ and $\mu_{l}=0$, and the investor never inspects. Thus the mis-aligned manager is more likely to adopt an uninformative system, and under the priors that the optimal system is informative, the optimal system is less informative and more 
liberal.

The equilibrium in the well-aligned manager's case is as follows. If $\bar{\mu}_{1} \leqslant \mu_{0}<\bar{\mu}_{2}$, the well-aligned manager's optimal system is uninformative and the investor inspects. If $\mu_{0}<\bar{\mu}_{1}$ or $\mu_{0}>\bar{\mu}_{2}$, the well-aligned manager's optimal system is fully-revealing, the investor does not inspect, and she approves the project upon seeing a $h$-signal and rejects upon seeing a $l$-signal. Information acquisition by the investor and by the manager's reporting system are substitutes when the incentives are well-aligned. 


\section{The Extended Model}

In the basic model of Chapter 2, the manager commits to disclosing the unaltered signal from the reporting system. This section relaxes this assumption and presents an extended model in which the manager privately observes the output of the system, and may manipulate the reporting system before it generates the signal to the investor. The manager considers his own future manipulation when designing the reporting system, so this case is equivalent to a partial commitment to the reporting system by the manager.

While this model is less tractable mathematically, it better captures the empirical finding that managers possess more information than they disclose, and may manipulate their disclosures to achieve a higher expected payoff. ${ }^{1}$

The purpose of the extended model is to answer three questions. First, from an ex-ante perspective, will a manager benefit from being able to manipulate the system ex post (i.e., after the signals are revealed by the system)? Second, how will ex-post manipulation affect the characteristics of the ex-ante optimal reporting system, and

\footnotetext{
1 Empirical evidence on earnings management suggests that managers manipulate the output of the reporting system (e.g., Burgstahler and Dichev (1997)). However, the scope of manipulation is constrained by regulation and other institutional features (e.g., Barton and Simko (2002)).
} 


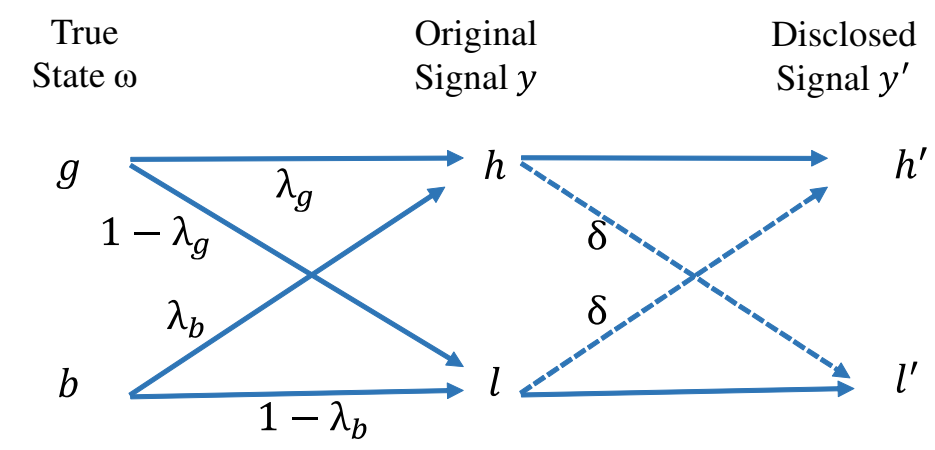

Figure 4.1: The Reporting System in the Extended Model

\begin{tabular}{|c|c|c|c|c|}
\hline$t=1$ & $t=2$ & $t=3$ & $t=4$ & $t=5$ \\
\hline $\begin{array}{l}\text { The manager chooses } \\
\text { an information system } \\
\text { for disclosure }\left(\lambda_{g}, \lambda_{b}\right) \text {. }\end{array}$ & $\begin{array}{l}\text { The manager observes } \\
\text { the output of the system } \\
y \text {, and chooses whether } \\
\text { to manipulate the system. }\end{array}$ & $\begin{array}{l}\text { Signal } y^{\prime} \text { is } \\
\text { disclosed. }\end{array}$ & $\begin{array}{l}\text { The investor first } \\
\text { decides whether to } \\
\text { inspect, and then } \\
\text { whether to invest. }\end{array}$ & Payoffs are realized. \\
\hline
\end{tabular}

Figure 4.2: The Timeline in the Extended Model

how will these characteristics change as the investor's information acquisition costs decrease? Third, how does allowing manipulation affect investment efficiency?

\subsection{Model Setup}

The setup of the extended model is as follows. After the reporting system is in place, the manager privately observes the reporting system's original signal $y \in\{h, l\}$, and chooses the probability of manipulation $\left(\phi_{y}\right)$. A manipulation can alter the output of the reporting system with probability $\delta$ at a fixed cost $F$. To differentiate, I denote the disclosed signal as $y^{\prime} \in\left\{h^{\prime}, l^{\prime}\right\}$. Both $\delta$ and $F$ are common knowledge. I allow managers with both high and low signals to manipulate, and assume $\delta\left(B+s N_{b}\right) \geqslant$ $F$, so the cost is sufficiently low for manipulation to occur. Figure 4.1 illustrates how manipulation affects the disclosed signal $\left(y^{\prime}\right)$, and Figure 4.2 presents the timeline of the extended model. 


\subsection{The equilibrium}

I study the Perfect Bayesian Equilibrium (PBE) of the extended model. The equilibrium in the extended model is comprised of the manager's choice of the reporting system $\left(\lambda_{g}, \lambda_{b}\right)$, the manager's manipulation strategy $\phi_{y}$, and the investor's response $a\left(\mu_{y^{\prime}}\right)$, such that:

1. At $t=1$, the manager chooses $\lambda_{g}, \lambda_{b}$ to maximize expected payoff:

$$
\left(\lambda_{g}, \lambda_{b}\right) \in \arg \max _{\lambda_{g}, \lambda_{b}} \mathbb{E}_{\pi\left(\lambda_{g}, \lambda_{b}\right)}\left(\mathbb{E}\left(\hat{v}\left(\mu_{y^{\prime}}\left(\phi_{h}, \phi_{l}\right)\right) \mid y\right)\right)
$$

2. At $t=2$, the manager privately observes the original signal $y$, and chooses his manipulation strategy $\left\{\phi_{h}, \phi_{l}\right\}$. Specifically,

(a) Upon receiving a $h$-signal, the manager chooses $\phi_{h} \in[0,1]$ to maximize expected payoff: $\phi_{h} \in \arg \max _{\phi} \mathbb{E}\left(\hat{v}\left(\mu_{y^{\prime}}\left(\phi, \phi_{l}\right)\right) \mid y=h\right)$.

(b) Upon receiving a $l$-signal, the manager chooses $\phi_{l} \in[0,1]$ to maximize expected payoff: $\phi_{l} \in \arg \max _{\phi} \mathbb{E}\left(\hat{v}\left(\mu_{y^{\prime}}\left(\phi_{h}, \phi\right)\right) \mid y=l\right)$.

3. At $t=4$, signal $y^{\prime}$ is disclosed. The investor chooses action (a) to maximize her expected payoff:

$$
a\left(\mu_{y^{\prime}}\right) \in \arg \max _{a} u\left(\mu_{y^{\prime}}\left(\phi_{h}, \phi_{l}\right), a\right), y^{\prime} \in\left\{h^{\prime}, l^{\prime}\right\}
$$

\subsection{Comparison with the no-manipulation case}

I first study whether allowing ex-post manipulation $(\delta>0)$ improves the manager's ex-ante expected payoff. $V_{\delta}\left(\mu_{0}\right)$ denotes the manager's maximum expected payoff from any reporting system when the scope of manipulation is $\delta$.

Lemma 4. $V_{\delta=0}\left(\mu_{0}\right) \geqslant V_{\delta>0}\left(\mu_{0}\right)$, regardless of the manager's type. 
Both the mis-aligned and the well-aligned managers achieve the maximum expected payoff when ex-post manipulation is outright forbidden. The proof is similar to that of the Revelation Principle (e.g., Arya et al. (1998)). Consider the optimal reporting system when $\delta>0$. In equilibrium, the manager may or may not manipulate the signal, but the investor will always interpret the signal rationally. The manager's expected payoff is determined by this distribution of the investor's posterior. There always exists a system under the no-manipulation case that generates the same distribution of posteriors, thus yielding the same gross expected payoff, but without incurring the fixed manipulation cost. As a result, a manager achieves the highest expected payoff when he designs and commits to the ex-ante optimal system.

\subsection{Well-aligned manager with ex-post manipulation}

Lemma 5. For a well-aligned manager, $V_{0}^{w}\left(\mu_{0}\right)=V_{\delta}^{w}\left(\mu_{0}\right), \forall \delta>0$. His optimal reporting system remains unaffected by allowing manipulation.

Lemma 5 established that a well-aligned manager's maximum payoff in the manipulation case will not exceed his payoff in the no-manipulation case. If a well-aligned manager's optimal system in the no-manipulation case yields the same expected payoff as in the manipulation case, this system must be optimal in the manipulation case as well. This is indeed the case. The optimal system from the no-manipulation case provides the well-aligned manager with no incentive to manipulate, since the investor will always take the manager's favored action. Manipulation is not only costly, but may also lead the investor to choose a less desirable action. Thus, allowing the wellaligned manager to manipulate the disclosed signal will not change his reporting system. 


\subsection{Mis-aligned manager with ex-post manipulation}

Clearly, when $\mu_{0} \geqslant \tilde{\mu}$, the mis-aligned manager's optimal system remains uninformative, since under such a system he has no incentive to manipulate and receives the same expected payoff as under the no-manipulation case. Thus, the following discussion focuses on the cases where the prior is pessimistic and the optimal system is informative. When $\mu_{0} \leqslant \tilde{\mu}$, his optimal system under no-manipulation yields a lower expected payoff and may no longer be optimal, since the possibility of manipulation makes the investor less willing to invest outright upon seeing the $h^{\prime}$-signal. The manager thus designs the optimal reporting system in anticipation of ex-post manipulation.

I derive the optimal system by solving first the subgame equilibrium after the reporting system is in place, and then solving the manager's choice of the optimal system. The intermediate lemmas and proofs are in Appendix A. Section 4.5.1 (4.5.2) presents the mis-aligned manager's optimal system, assuming high (low) investor inspection costs.

\subsubsection{Mis-aligned manager and high investor inspection costs}

The following proposition characterizes the optimal system in the manipulation case when the investor faces high inspection costs $(m \geqslant \bar{m})$.

Proposition 3. Suppose $m>\bar{m}$ and $\mu_{0}<\bar{\mu}$.

$$
\text { Denote } \bar{f}_{1}=\frac{\left(B+s N_{g}\right)+\frac{1-\bar{\mu}}{\bar{\mu}}\left(B+s N_{b}\right)}{\frac{1-\mu_{0}}{\mu_{0}}\left(1-\tilde{\lambda}_{b}\right)+\frac{1}{\delta\left(B+s N_{b}\right)}\left(\left(B+s N_{g}\right)+\frac{1-\bar{\mu}}{\bar{\mu}}\left(B+s N_{b}\right)\right)} \text {, where } \tilde{\lambda}_{b}=\frac{1}{1-\delta}\left(\frac{1-\bar{\mu}}{\bar{\mu}} \frac{\mu_{0}}{1-\mu_{0}}-\delta\right) \text {. }
$$

The mis-aligned manager's optimal reporting system is as follows:

1. If $\delta>\frac{\mu_{0}}{1-\mu_{0}} \frac{1-\bar{\mu}}{\bar{\mu}}$ or $F>\bar{f}_{1}$ : The optimal system $\left(\pi_{1}\right)$ has $\lambda_{g}=1, \lambda_{b}=\frac{\mu_{0}}{1-\mu_{0}} \frac{1-\bar{\mu}}{\bar{\mu}}$. The manager does not manipulate $\left(\phi_{h}=\phi_{l}=0\right)$. The investor's posteriors are $\mu_{h^{\prime}}=\bar{\mu}$ and $\mu_{l^{\prime}}=0$. Upon seeing the $h^{\prime}$-signal, the investor approves outright 
with probability $\chi_{h^{\prime}}=\frac{F}{\delta\left(B+s N_{b}\right)}$, and rejects outright with probability $1-\chi_{h^{\prime}}$.

2. If $\delta \leqslant \frac{\mu_{0}}{1-\mu_{0}} \frac{1-\bar{\mu}}{\bar{\mu}}$ and $F \leqslant \bar{f}_{1}$ : The optimal system $\left(\pi_{2}\right)$ has $\lambda_{g}=1, \lambda_{b}=$ $\frac{1}{1-\delta}\left(\frac{\mu_{0}}{1-\mu_{0}} \frac{1-\bar{\mu}}{\bar{\mu}}-\delta\right)$. The l-signal manager manipulates and the $h$-signal manager does not $\left(\phi_{h}=0\right.$ and $\left.\phi_{l}=1\right)$. The investor's posteriors are $\mu_{h^{\prime}}=\bar{\mu}$ and $\mu_{l^{\prime}}=0$. Upon seeing the $h^{\prime}$-signal, the investor approves outright with probability $\chi_{h^{\prime}}=1$.

Proposition 3 shows that the optimal system under the no-manipulation case (System $\pi_{1}$ ) remains optimal when either the scope $(\delta)$ or the cost $(F)$ of manipulation is large. In that case, under System $\pi_{1}$, the original output of the reporting system $y$ gives posteriors $\mu_{h}=\bar{\mu}$ and $\mu_{l}=0$. In equilibrium, the manager does not manipulate, and thus upon seeing the disclosed signals $y^{\prime}$, the investor's posteriors remain $\mu_{h^{\prime}}=\bar{\mu}$ and $\mu_{l^{\prime}}=0$, the same as in the no-manipulation case. However, her response differs: Upon seeing the $h^{\prime}$-signal, she invests without inspection with probability $\chi_{h^{\prime}}=\frac{F}{\delta\left(B+s N_{b}\right)}<1$, and rejects with probability $1-\chi_{h^{\prime}}$. She chooses this mixed strategy to make the manager indifferent between manipulating or not. As a result, the manager does not manipulate in equilibrium. Compared to the nomanipulation case where the investor invests with certainty upon seeing a $h$-signal, allowing manipulation hurts the manager's expected payoff through the reduced investment.

When both the scope and cost of manipulation are small, the optimal system becomes $\pi_{2}$, which has a lower $\lambda_{b}$ and is more informative than System $\pi_{1}$ ex ante. Under $\pi_{2}$, the manager with a $l$-signal always manipulates. However, although manipulation makes the $h^{\prime}$-signal less indicative of good state, a $h^{\prime}$-signal still convinces the investor to provide outright approval: The investor's posteriors are $\mu_{h^{\prime}}\left(\phi_{l}=1\right)=\bar{\mu}$ and $\mu_{l^{\prime}}\left(\phi_{l}=1\right)=0$. Thus, the posteriors are the same as in the no-manipulation 


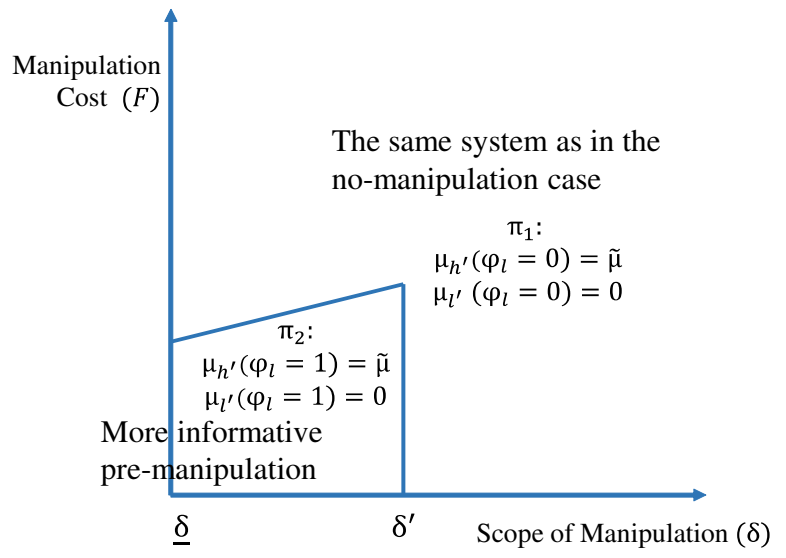

Figure 4.3: Properties of the Optimal Reporting System when Manipulation is Allowed

case; what differs is that the pre-manipulation system needs to be more informative, and the manager incurs manipulation costs.

The intuition for the tradeoff between the two systems is as follows. System $\pi_{2}$ is optimal only if $\delta$ is small. Otherwise, if the scope of manipulation is large, regardless of $\lambda_{b}$, the investor will not trust a $h^{\prime}$-signal enough for outright approval. When $\delta$ is small, both systems are potentially optimal. System $\pi_{1}$ reduces the likelihood of investment, while $\pi_{2}$, a more informative system, increases the manipulation incentive for the $l$-signal manager and results in higher manipulation cost. The optimal system thus depends on the relative costs of manipulation versus foregone investment. When manipulation cost $F$ is small, System $\pi_{2}$ accommodates manipulation at a low cost and is thus preferred; otherwise, System $\pi_{1}$ forgoes a small percentage of investment $\left(\chi_{h}\right.$ increases in $\left.F\right)$ and is preferred. Figure 4.3 shows the optimal system under different $\delta$ and $F$ values.

\subsubsection{Mis-aligned manager and low investor inspection costs}

Relative to a setting in which high inspection costs preclude investor information acquisition, the low inspection cost setting offers the manager an advantage, in that 
he can choose a reporting system that encourages investor inspection. The following proposition characterizes the optimal system when the market prior is pessimistic $\left(\mu_{0} \leqslant \bar{\mu}_{1}\right)$, under which the optimal system is informative. The optimal system and the subgame equilibrium are as follows.

Proposition 4. Suppose $m<\bar{m}$ and $\mu_{0}<\bar{\mu}_{1}$. Denote $\bar{f}_{2}=\frac{\frac{1-\bar{\mu}_{2}}{\bar{\mu}_{2}} \delta\left(B+s N_{b}\right)}{\frac{1-\bar{\mu}_{2}}{\bar{\mu}_{2}}+\frac{1-\mu_{0}}{\mu_{0}}\left(1-\lambda_{b, 2}\right) \delta}$, where $\lambda_{b, 2}=\frac{1}{1-\delta}\left(\frac{1-\bar{\mu}_{2}}{\bar{\mu}_{2}} \frac{\mu_{0}}{1-\mu_{0}}-\delta\right)$. The mis-aligned manager's optimal reporting system is as follows:

1. If $\delta>\frac{\mu_{0}}{1-\mu_{0}} \frac{1-\bar{\mu}_{2}}{\bar{\mu}_{2}}$ or $F>\bar{f}_{2}$ : The optimal system $\left(\pi_{1}\right)$ has $\lambda_{g}=1, \lambda_{b}=\frac{\mu_{0}}{1-\mu_{0}} \frac{1-\bar{\mu}_{2}}{\bar{\mu}_{2}}$. The manager does not manipulate $\left(\phi_{h}=\phi_{l}=0\right)$. The investor's posteriors are $\mu_{h^{\prime}}=\bar{\mu}_{2}$ and $\mu_{l^{\prime}}=0$. She approves without inspection upon seeing a $h^{\prime}$-signal with probability $\chi_{h^{\prime}}=\frac{F}{\delta\left(B+s N_{b}\right)}$, and inspects with probability $1-\chi_{h^{\prime}}$.

2. If $\delta \leqslant \frac{\mu_{0}}{1-\mu_{0}} \frac{1-\bar{\mu}_{2}}{\bar{\mu}_{2}}$ and $F \leqslant \bar{f}_{2}$ : The optimal system $\left(\pi_{2}\right)$ has $\lambda_{g}=1, \lambda_{b}=$ $\frac{1}{1-\delta}\left(\frac{\mu_{0}}{1-\mu_{0}} \frac{1-\bar{\mu}_{2}}{\bar{\mu}_{2}}-\delta\right)$. The l-signal manager manipulates and the h-signal manager does not $\left(\phi_{h}=0\right.$ and $\left.\phi_{l}=1\right)$. The investor's posteriors are $\mu_{h^{\prime}}=\bar{\mu}_{2}$ and $\mu_{l^{\prime}}=0$, and she approves without inspection upon seeing a $h^{\prime}$-signal with probability $\chi_{h^{\prime}}=1$.

When investor inspection cost is low and the market prior is pessimistic $\left(\mu_{0}<\bar{\mu}_{1}\right)$, the optimal system is the same as under the no-manipulation case (System $\pi_{1}$ ) when the scope $(\delta)$ or the cost $(F)$ of manipulation is large. Otherwise, the optimal system is the more informative System $\pi_{2}$. When the market prior is in the intermediate range $\left(\bar{\mu}_{1} \leqslant \mu_{0}<\bar{\mu}_{2}\right)$, the potential optimal systems are $\pi_{1}, \pi_{2}$, and another system $\pi_{3}$ that yields $\mu_{h^{\prime}}=\bar{\mu}_{2}$ and $\bar{\mu}_{1} \leqslant \mu_{l^{\prime}}<\mu_{0}$, under which the manager does not manipulate $\left(\phi_{h}=\phi_{l}=0\right)$, the investor inspects upon receiving a $l^{\prime}$-signal, and approves 
outright upon seeing a $h^{\prime}$-signal with probability $\chi_{h^{\prime}}=\frac{F}{\delta\left(1-\mu_{l^{\prime}}\right)\left(B+s N_{b}\right)}$. I draw three conclusions from these results.

Corollary 4. The impact of $\delta$ and $F$ on the mis-aligned manager's expected payoff is as follows:

1. $V_{\delta}^{m}\left(\mu_{0}\right)$ weakly decreases in $\delta$;

2. When $\delta<\frac{\mu_{0}}{1-\mu_{0}} \frac{1-\tilde{\mu}}{\tilde{\mu}}, V_{\delta}^{m}\left(\mu_{0}\right)$ weakly decreases (increases) in $F$ when $F$ is sufficiently small (large).

Corollary 4 shows a mis-aligned manager's expected payoff decreases monotonically in the scope of manipulation $(\delta)$, but changes non-monotonically with the manipulation cost $F$. When $F$ is small, the cost of manipulation is low and the manager prefers the more informative System $\pi_{2}$, under which a manager with $l$-signal manipulates the signal. As a result, the ex-ante expected payoff decreases in F. However, when $F$ is sufficiently big, the manager chooses System $\pi_{1}$, as under the no-manipulation case. Upon seeing a $h^{\prime}$-signal, the investor invests outright with a probability $\chi_{h^{\prime}}$ that increases in $F$. As a result, $V_{\delta}^{m}\left(\mu_{0}\right)$ decreases in $F$ when $F$ is sufficiently large.

Corollary 5. Suppose $\mu_{0}<\bar{\mu}_{1}$. As $m$ decreases, the post-manipulation signal $y^{\prime} \in$ $\left(h^{\prime}, l^{\prime}\right)$ becomes less liberal.

Similar to the no-manipulation case, the post-manipulation signal $y^{\prime}$ is less liberal as investor inspection costs decrease.

Corollary 6. Suppose $m<\bar{m}$ and $\mu_{0}<\bar{\mu}_{1}$. Firms with a mis-aligned manager over-invest in bad projects, and over-investment deceases in $\delta$.

While a larger scope of manipulation reduces the mis-aligned manager's expected payoff, it improves investment efficiency when investor inspection costs are low. A 
larger $\delta$ leads to more ex-post manipulation, and makes the investor more suspicious towards the $h^{\prime}$-signal. As a result, the investor approves outright less $\left(\chi_{h^{\prime}}=\frac{F}{\delta\left(B+s N_{b}\right)}\right)$ and inspects more, which results in less over-investment and higher efficiency. The intuition is that the commitment to disclose the unaltered signal benefits the manager because it convinces the investor to invest according to the positively biased system. A larger $\delta$ weakens this commitment and leads the investor to rely more on inspection, which improves investment efficiency.

Corollary 6 speaks to an unintended benefit of managerial discretion over disclosures. When the reporting system is endogenous, a commitment not to manipulate enables the manager to adopt reporting systems that discourage investor information acquisition, leading to over-investment in bad projects. Thus, when investor inspection costs are low, regulations that aim to soften such commitment will improve investment efficiency.

To summarize, a well-aligned manager's optimal reporting system under the nomanipulation case remains optimal when manipulation is allowed, while a mis-aligned manager's may no longer be optimal. The mis-aligned manager chooses the more informative System $\pi_{2}$ when both the scope and the cost of manipulation are small. The investor's posterior beliefs upon seeing the signals are the same as under the nomanipulation case, but her investment may be less responsive to the signals. As $m$ decreases, the post-manipulation signals become more informative. In Appendix B, Table B.1 presents the optimal system for each case when manipulation is forbidden (the basic model), and Table B.2 presents the optimal system when manipulation is allowed. 


\section{5 \\ Empirical Implications}

By relaxing certain assumptions in prior theoretical models of disclosure, my model generates predictions that are both unavailable from prior literature and consistent with empirical findings. At the same time, I incorporate two key determinants of disclosures identified in empirical literature (managerial incentive alignment and market prior beliefs about project profitability), and examine their effects on disclosure for all possible combinations of determinants. Combined, the analysis generates alternative interpretations for several empirical findings as well as new empirical implications. First, it shows that both mis-aligned and well-aligned managers will voluntarily disclose bad news, as part of the optimally designed information system. This prediction is not available from the large class of disclosure models that view disclosure as an ex-post choice by managers (e.g., Verrecchia (1983), Jung and Kwon (1988)). It also suggests that bad news disclosures are not necessarily situationor event-driven (Skinner (1994)), and are not necessarily indicative of managerial incentive mis-alignment (Aboody and Kasznik (2000)) or alignment (Kumar et al. (2012)).

Further, my model predicts that good and bad news disclosures can differ in their 
informativeness, a prediction consistent with empirical findings (e.g., Kasznik and Lev (1995), Kothari et al. (2009)), but unavailable from models that view ex-ante disclosure strategy as choosing only the precision of an additive, normally-distributed noise term (e.g., Diamond and Verrecchia (1991)). ${ }^{1}$

Second, while prior studies have considered the impact of investors' private information on firms' disclosures (Dye and Sridhar (2002), Gao and Liang (2013), Zuo (2013)), they are silent on how the effect depends on managerial incentives. My model shows that only well-aligned managers have incentives to design the reporting system so as to motivate investor information acquisition. Furthermore, the optimal system differs depending on investors' information acquisition cost. Specifically, my analysis predicts that firms with well-aligned managers are more likely to offer informative disclosures when investors' inspection cost is high, consistent with findings in Balakrishnan et al. (2014) and Chen and Vashishtha (2015) that firms increase disclosures after experiencing loss of analyst coverage or loss of bank monitoring, to the extent that these events make it more difficult for investors to obtain additional information.

My analysis also predicts that when investors' information acquisition cost is low, a well-aligned manager's reporting exhibits counter-cyclicality, consistent with empirical evidence that managers smooth earnings (e.g., Liu and Ryan (2006)). Under this interpretation, my analysis offers an alternative explanation for Tucker and Zarowin's (2006) finding of a positive association between earnings smoothness and the ability of stock price to predict future earnings. Tucker and Zarowin (2006) interpret this finding as consistent with managers smoothing earnings to improve earnings informativeness about future performance. My model suggests that well-aligned managers smooth earnings to reduce earnings' informativeness, which motivates investors to

${ }^{1}$ An exception is Armstrong et al. (ming), in which good and bad news are allowed to have different levels of precision. 
acquire additional information. Nonetheless, since the acquired information affects firms' investment decisions and thus future cash flows, and at the same time is likely to be impounded in stock price, stock prices in firms with smoother earnings are expected to be more positively associated with future earnings. ${ }^{2}$

More importantly, the finding that counter-cyclical reporting is chosen by wellaligned managers cautions against interpreting earnings smoothing as evidence of manipulation by mis-aligned managers (e.g., Leuz et al. (2003), Jayaraman (2008)). Specifically, my analysis identifies that such an interpretation presumes that investors are unable to acquire additional information, or unable to intervene in firms' decisions, or both. When these assumptions are violated, ${ }^{3}$ earnings smoothness indicates better incentive alignment between managers and investors. More generally, my analysis shows that under certain conditions, (e.g., when investors' inspection costs are low and the market prior is intermediate or low), mis-aligned managers issue more informative disclosures than well-aligned managers.

Third, my analysis suggests an alternative explanation for the positive association between informational properties of disclosures and investment efficiency (e.g., Biddle et al. (2009), Francis and Martin (2010)). While some interpret such an association as consistent with certain reporting properties (such as conservatism) disciplining managers' over-investment, my analysis shows that such a positive relation can also exist as a result of cross-sectional differences in investors' inspection

2 This result is similar to Gao and Liang (2013), who find that a value-maximizing managers may reduce public disclosure to motivate information acquisition by traders. My model differs from theirs in two dimensions: First, investors in my model decide whether to acquire additional information after observing firm's disclosure; second, I allow managers to use both informativeness and bias to induce investor information acquisition.

${ }^{3}$ It is an empirical question whether and to what extent reported earnings affect investors' decisions and in turn affect firms' future decisions and cash flows. Dechow et al. (2010) note that, outside the U.S., earnings smoothing is associated with low earnings quality and poor shareholder rights (Leuz et al. (2003)); within the U.S., earnings smoothing is associated with more informative stock prices (Tucker and Zarowin (2006)). My analysis reconciles these findings by tracing them to U.S. investors having lower information acquisition costs and U.S. managers' incentives being better-aligned with those of shareholders. 
costs. Specifically, I show that investment efficiency tends to be high among firms whose investors' inspection costs are low, and at the same time, mis-aligned managers reduce the liberal bias in their reporting (i.e., accounting appears more conservative) when investors' inspection costs are low. While some researchers hypothesize that the relation between reporting properties and investment efficiency may not be causal (see Dechow et al. (2010), Roychowdhury $(2010)^{4}$ ), my analysis specifically identifies investors' inspection costs as a correlated omitted variable.

Fourth, I analyze how investors' cost of acquiring additional information affects the properties of firms' disclosures. This issue is of concern to both standard setters and academic researchers, but has received little attention in analytical research so far. As a result, consensus is lacking on whether and how the properties of public disclosures are affected by the changing landscape of investor information acquisition. While some scholars deplore what they see as the diminishing relevance of financial accounting information due to competition with other information sources (Lev and Zarowin (1999)), others note that the informativeness of accounting information is not eroded by at least one form of competing information (Francis et al. (2002a)), or that accounting information has become more conservative (Givoly and Hayn (2000)). My analysis identifies managerial incentive alignment as a key determinant for the relation between disclosure properties and investors' information acquisition costs. As these costs decrease, disclosures will become more (less) informative if disclosures are mostly driven by mis-aligned (well-aligned) managers. Furthermore, to the extent that mis-aligned managers exist in the sample, reported earnings will exhibit less positive bias (that is, appear more conservative) over time.

Lastly, my analysis of the extended model that expressly allows ex-post manipulation by the manager demonstrates the value of commitment to ex-ante optimal

\footnotetext{
${ }^{4}$ Roychowdhury (2010) points out in the abstract: "The evidence [in Francis and Martin (2010)] is insufficient to infer a casual relation between conservatism and acquisition profitability."
} 
disclosure strategies (e.g., Chen et al. (F)). This analysis further suggests that misaligned managers have stronger incentives to seek out costly external mechanisms (auditors, credit rating agencies) to establish "reporting reputation" in that they benefit more from credibly committing not to engage in costly ex-post manipulation. At the same time, my analysis reveals that the equilibrium informativeness of disclosure is not changed, possibly explaining the difficulty in detecting the effect of auditor reputation on reporting quality (see Lawrence et al. (2011), DeFond et al. (2015), and DeFond and Zhang (2014) for a survey). In addition, my analysis adds to the literature that discovers benefits of managerial manipulation in certain settings (e.g., Dutta and Gigler (2002), Jiang and Xin (2015)). I show that a larger scope of ex-post manipulation can actually improve investment efficiency in firms with mis-aligned managers and low investor inspection costs, because investors will be more cautious and more likely to acquire additional information.

In summary, my analysis highlights that both the nature of disclosed information and investors' response to it matter for understanding firms' strategic disclosure behavior. A key condition for my results is that the disclosed information is forwardlooking in nature and relevant to the firms' investment opportunities, which affects investors' assessment of firms' prospects, and ultimately changes their decisions and alters firms' future cash flows. 
6

\section{Conclusion}

This paper analyzes how managers design the reporting systems for forward-looking information about firms' investment opportunities, in anticipation of investors' information acquisition and intervention decisions. In the basic model, I assume the manager must truthfully disclose the signal generated by the system. In the extended model, I allow the manager to decide whether to manipulate the signal prior to its disclosure.

My basic model highlights that investor information acquisition serves different roles for well-aligned managers and mis-aligned managers. For the former, investor information acquisition leads to more informed investment decisions. For the latter, on the other hand, investor information acquisition deters inefficient investment in bad projects. Incentive alignment is a prerequisite for managers to benefit from investors' information acquisition. Furthermore, the model predicts that, if incentives are mis-aligned, lower investor inspection costs lead to more informative and less liberal reporting systems; when incentives are well-aligned, lower investor inspection costs lead to less informative systems, but the change in bias depends on the market prior. In the past decades, investor information acquisition cost has decreased 
substantially, which explains the findings of a concurrent trend in the expansion of corporate disclosures (to the extent that incentive mis-alignment is present in the sample), and more importantly, also speaks to the increase in accounting conservatism. The results highlight the complex interactions among three key determinants of the optimal reporting system: the cost of investor information acquisition, incentive alignment between the manager and the representative investor, and the commonly-held prior belief about the investment opportunity.

The paper provides alternative explanations for several extant empirical results. For example, it shows that both mis-aligned and well-aligned managers voluntarily disclose bad news, as part of the optimally designed information system. In addition, the positive associations between the properties of disclosure (informativeness and bias) and investment efficiency can be a result of cross-sectional differences in investors' inspection costs, and earnings smoothing behavior can be chosen by wellaligned managers to induce investors' information acquisition. Mis-aligned managers have stronger incentives to manipulate ex post, but also have stronger incentives to seek out external mechanisms to credibly commit not to manipulate. My model also generates yet-untested predictions for how disclosures are optimally structured under various conditions. For example, it identifies conditions under which mis-aligned managers issue more informative disclosures than well-aligned managers, and predicts how the informativeness and bias in disclosures vary across the business cycle. Overall, a key insight from my analysis is that the nature of the disclosed information and how investors are expected to respond to that information matter for understanding firms' disclosure behavior. 


\title{
Appendix A
}

\author{
Proofs
}

\section{A.1 Proof of Lemma 1}

Proof. Let $\bar{\mu}_{1}$ denote the posterior belief that the state is $\omega=g$, such that the investor is indifferent between rejecting and inspecting before investing, i.e., $(1-s) \bar{\mu}_{1} N_{g}-$ $m=0$, which yields $\bar{\mu}_{1}=\frac{m}{(1-s) N_{g}}$. When $\mu>\bar{\mu}_{1}$, the investor strictly prefers inspection over outright rejection. Similarly, let $\bar{\mu}_{2}$ be the posterior belief about state $\omega=g$ such that the investor is indifferent between investing directly and inspecting before investing, i.e., $(1-s) \bar{\mu}_{1} N_{g}-m=(1-s)\left(\mu N_{g}+(1-\mu) N_{b}\right)$, which yields $\bar{\mu}_{2}=1-\frac{m}{(1-s)\left|N_{b}\right|}$. When $\mu<\bar{\mu}_{2}$, the investor strictly prefers inspecting over investing directly. Finally define $\bar{\mu}$ as the posterior belief about state $\omega=g$ such that the investor is indifferent between directly rejecting and directly investing, i.e., $(1-s)\left(\mu N_{g}+(1-\mu) N_{b}\right)=0$, which yields $\bar{\mu}=\frac{\left|N_{b}\right|}{\left|N_{b}\right|+N_{g}}$. The investor strictly prefers investing to rejecting when $\mu>\bar{\mu}$.

Inspecting before investing is optimal only when $\bar{\mu}_{1}<\mu<\bar{\mu}_{2}$, which implies that $\bar{\mu}_{1}<\bar{\mu}_{2}$. Let $\bar{m}$ be the threshold inspection cost that equates $\bar{\mu}_{1}=\bar{\mu}_{2}$. Rearranging $\frac{m}{(1-s) N_{g}}<1-\frac{m}{(1-s)\left|N_{b}\right|}$ yields $\bar{m}=\frac{(1-s) N_{g}\left|N_{b}\right|}{\left|N_{b}\right|+N_{g}}$. Thus, when $m \geqslant \bar{m}$, the investor will 
approve outright without inspection if $\mu \geqslant \bar{\mu}$ and will reject outright if $\mu<\bar{\mu}$. When $m<\bar{m}$, the investor will inspect and invest only in good projects if $\bar{\mu}_{1}<\mu<\bar{\mu}_{2}$; the investor will reject outright if $\mu<\bar{\mu}_{1}$, and approve outright if $\mu>\bar{\mu}_{2}$.

\section{A.2 Proof of Proposition 1}

Proof. I discuss the cases $m \geqslant \bar{m}$ and $m<\bar{m}$ separately.

1. When $m \geqslant \bar{m}$, the investor does not inspect.

(a) The case where $\mu_{0} \geqslant \bar{\mu}$ : Consider any Bayesian plausible posterior $\mu_{h}$ and $\mu_{l}$. The manager's expected payoff under this system is:

$$
\begin{aligned}
\mathbb{E}(\hat{v}(\mu)) & =\mathbb{E}\left(\mathbf{1}(\mu \geqslant \bar{\mu})\left(B+s\left(\mu N_{g}+(1-\mu) N_{b}\right)\right)\right) \\
& =\mathbb{E}\left(\mathbf{1}(\mu \geqslant \bar{\mu}) \mu\left(B+s N_{g}\right)\right)+\mathbb{E}\left(\mathbf{1}(\mu \geqslant \bar{\mu})(1-\mu)\left(B+s N_{b}\right)\right) \\
& \leqslant \mathbb{E}\left(\mu\left(B+s N_{g}\right)\right)+\mathbb{E}\left((1-\mu)\left(B+s N_{b}\right)\right)=\hat{v}\left(\mu_{0}\right)
\end{aligned}
$$

Thus, the uninformative system gives the highest expected payoff. Since any informative system incurs a positive cost, the uninformative system is optimal.

(b) If $0<\mu_{0}<\bar{\mu}$ : First, if the optimal system is informative, it must have $\mu_{h} \geqslant \bar{\mu}$. Otherwise, $\mathbb{E}(\hat{v}(\mu))=\operatorname{Pr}\left(\mu_{h}\right) \hat{v}\left(\mu_{h}\right)+\operatorname{Pr}\left(\mu_{l}\right) \hat{v}\left(\mu_{l}\right)=0$, the same as the payoff under the uninformative system. Second, the optimal system must have $\mu_{l}=0$. If not, assume the optimal system $\pi$ has $\mu_{l}>0$ and $\mu_{h} \geqslant \bar{\mu}$. Then consider an alternative system $\pi^{\prime}$ with $\mu_{l}=0$ and $\mu_{h} \geqslant \bar{\mu}$. Since $\mu_{0}=\operatorname{Pr}\left(\mu_{h} \mid \pi\right) \mu_{h}+\operatorname{Pr}\left(\mu_{l} \mid \pi\right) \mu_{l}=\operatorname{Pr}\left(\mu_{h} \mid \pi^{\prime}\right) \mu_{h}$, we have $\operatorname{Pr}\left(\mu_{h} \mid \pi^{\prime}\right)>\operatorname{Pr}\left(\mu_{h} \mid \pi\right)$; that is, the probability of generating a high signal $h$ is higher under $\pi^{\prime}$.

$$
\mathbb{E}_{\pi\left(\mu_{h}, \mu_{l}\right)}(\hat{v}(\mu))=\operatorname{Pr}\left(\mu_{h} \mid \pi\right) \hat{v}\left(\mu_{h}\right)<\operatorname{Pr}\left(\mu_{h} \mid \pi^{\prime}\right) \hat{v}\left(\mu_{h}\right)=\mathbb{E}_{\pi\left(\mu_{h}, 0\right)}(\hat{v}(\mu))
$$


Finally, the optimal system must have $\mu_{h}=\bar{\mu}$. Suppose this is not the case; then assume $\mu_{l}=0$ and $\mu_{h}>\bar{\mu}$. Consider an alternative system $\pi^{\prime}$ with $\mu_{l}=0$ and $\mu_{h}=\bar{\mu}$.

$$
\begin{aligned}
\mathbb{E}_{\pi\left(\mu_{h}, 0\right)}(\hat{v}(\mu)) & =\operatorname{Pr}\left(\mu_{h} \mid \pi\right) \hat{v}\left(\mu_{h}\right) \\
& =\operatorname{Pr}\left(\mu_{h} \mid \pi\right) B+\operatorname{Pr}\left(\mu_{h} \mid \pi\right)\left(s \mu_{h} N_{g}+s\left(1-\mu_{h}\right) N_{b}\right) \\
& <\operatorname{Pr}\left(\mu_{h} \mid \pi^{\prime}\right) B+\left(s \mu_{0} N_{g}+s\left(1-\mu_{0}\right) N_{b}\right)=\mathbb{E}_{\pi(\bar{\mu}, 0)}(\hat{v}(\mu))
\end{aligned}
$$

So, the optimal reporting system has posterior beliefs $\mu_{l}=0$ and $\mu_{h}=\bar{\mu}$. Solving for $\lambda_{g}$ and $\lambda_{b}$ yields $\lambda_{g}=1$ and $\lambda_{b}=\frac{\mu_{0}}{1-\mu_{0}} \frac{1-\bar{\mu}}{\bar{\mu}}$.

2. When $m<\bar{m}$ :

(a) The case $\mu_{0} \geqslant \bar{\mu}_{2}$ : The manager achieves the highest expected payoff under an uninformative system:

$$
\begin{aligned}
& \mathbb{E}(\hat{v}(\mu)) \\
= & \mathbb{E}\left(\mathbf{1}\left(\bar{\mu}_{2}>\mu \geqslant \bar{\mu}_{1}\right) \mu\left(B+s N_{g}\right)\right)+\mathbb{E}\left(\mathbf{1}\left(\mu \geqslant \bar{\mu}_{2}\right)\left(B+s\left(\mu N_{g}+(1-\mu) N_{b}\right)\right)\right) \\
= & \mathbb{E}\left(\mathbf{1}\left(\mu \geqslant \bar{\mu}_{1}\right) \mu\left(B+s N_{g}\right)\right)+\mathbb{E}\left(\mathbf{1}\left(\mu \geqslant \bar{\mu}_{2}\right)\left(B+s N_{b}\right)\right) \\
\leqslant & \mathbb{E}\left(\mu\left(B+s N_{g}\right)\right)+\mathbb{E}\left((1-\mu)\left(B+s N_{b}\right)\right) \\
= & B+s\left(\mu_{0} N_{g}+\left(1-\mu_{0}\right) N_{b}\right)=\hat{v}\left(\mu_{0}\right)
\end{aligned}
$$

Since any informative system incurs a positive cost, the optimal system is uninformative, with $\mu_{h}=\mu_{l}=\mu_{0}$.

(b) The case $\bar{\mu}_{1}<\mu_{0}<\bar{\mu}_{2}$ : First, if the optimal system is informative, it must have $\mu_{h} \geqslant \bar{\mu}_{2}$. Otherwise, the manager's expected payoff will be no higher than with the uninformative system, $\hat{v}\left(\mu_{0}\right)$ :

$$
\begin{aligned}
& \mathbb{E}(\hat{v}(\mu)) \\
= & \mathbb{E}\left(\mathbf{1}\left(\bar{\mu}_{2}>\mu \geqslant \bar{\mu}_{1}\right) \mu\left(B+s N_{g}\right)\right)+\mathbb{E}\left(\mathbf{1}\left(\mu \geqslant \bar{\mu}_{2}\right)\left(B+s\left(\mu N_{g}+(1-\mu) N_{b}\right)\right)\right) \\
\leqslant & \mathbb{E}\left(\mu\left(B+s N_{g}\right)\right)=\mu_{0}\left(B+s N_{g}\right)=\hat{v}\left(\mu_{0}\right)
\end{aligned}
$$


Second, the optimal system must have $\mu_{l}=0$. Otherwise, assume the optimal system $\pi$ has $\mu_{l}>0$ and $\mu_{h} \geqslant \bar{\mu}_{2}$. An alternative system $\pi^{\prime}$ with the same $\mu_{h}$ and $\mu_{l}=0$ will give a higher expected payoff for any $\mu_{l} \leqslant \mu_{0}<\bar{\mu}_{2}$

If $\mu_{l}<\bar{\mu}_{1}$ :

$$
\mathbb{E}_{\pi\left(\mu_{h}, \mu_{l}\right)}(\hat{v}(\mu))=<\operatorname{Pr}\left(\mu_{h} \mid \pi\right) \hat{v}\left(\mu_{h}\right)<\operatorname{Pr}\left(\mu_{h} \mid \pi^{\prime}\right) \hat{v}\left(\mu_{h}\right)=\mathbb{E}_{\pi\left(\mu_{h}, 0\right)}(\hat{v}(\mu))
$$

If $\bar{\mu}_{1} \leqslant \mu_{l}<\bar{\mu}_{2}$ :

$$
\begin{aligned}
\mathbb{E}_{\pi\left(\mu_{h}, \mu_{l}\right)}(\hat{v}(\mu)) & =\operatorname{Pr}\left(\mu_{l} \mid \pi\right) \hat{v}\left(\mu_{l}\right)+\operatorname{Pr}\left(\mu_{h} \mid \pi\right) \hat{v}\left(\mu_{h}\right) \\
& =\mu_{0}\left(B+s N_{g}\right)+\operatorname{Pr}\left(\mu_{h} \mid \pi\right)\left(1-\mu_{h}\right)\left(B+s N_{b}\right) \\
& <\mathbb{E}_{\pi\left(\mu_{h}, 0\right)}(\hat{v}(\mu))
\end{aligned}
$$

Third, the optimal system must have $\mu_{h}=\bar{\mu}_{2}$. If this is not the case, assume $\mu_{l}=0$ and $\mu_{h}>\bar{\mu}_{2}$. Then an alternative system $\pi^{\prime}$ with $\mu_{l}=0$ and $\mu_{h}=\bar{\mu}_{2}$ will give a higher expected payoff:

$$
\begin{aligned}
\mathbb{E}_{\pi\left(\mu_{h}, 0\right)}(\hat{v}(\hat{\mu})) & =\operatorname{Pr}\left(\mu_{h} \mid \pi\right) B+s \operatorname{Pr}\left(\mu_{h} \mid \pi\right)\left(\mu_{h} N_{g}+\left(1-\mu_{h}\right) N_{b}\right) \\
& <\operatorname{Pr}\left(\bar{\mu}_{2} \mid \pi^{\prime}\right) B+s\left(\mu_{0} N_{g}+\left(1-\mu_{0}\right) N_{b}\right)=\mathbb{E}_{\pi\left(\bar{\mu}_{2}, 0\right)}(\hat{v}(\mu))
\end{aligned}
$$

Solving for $\lambda_{g}$ and $\lambda_{b}$ yields $\lambda_{g}=1$ and $\lambda_{b}=\frac{\mu_{0}}{1-\mu_{0}} \frac{1-\bar{\mu}_{2}}{\bar{\mu}_{2}}$.

(c) The case $0<\mu_{0}<\bar{\mu}_{1}$ : First, the optimal system is informative and has $\mu_{h} \geqslant \bar{\mu}_{1}$. When $\mu_{h}>\bar{\mu}_{1}$ and $\operatorname{Pr}(h \mid \pi)>0$, the manager's expected payoff is positive. Otherwise, his expected payoff is 0 . Second, $\mu_{l}=0$. Otherwise, assume the optimal system has $\mu_{l}>0$ and $\mu_{h} \geqslant \bar{\mu}_{1}$. Then consider an alternative system $\pi^{\prime}$ with $\mu_{l}=0$ and $\mu_{h}$ :

$$
\mathbb{E}_{\pi\left(\mu_{h}, \mu_{l}\right)}(\hat{v}(\mu))=\operatorname{Pr}\left(\mu_{h} \mid \pi\right) \hat{v}\left(\mu_{h}\right)<\operatorname{Pr}\left(\mu_{h} \mid \pi^{\prime}\right) \hat{v}\left(\mu_{h}\right)=\mathbb{E}_{\pi\left(\mu_{h}, 0\right)}(\hat{v}(\mu))
$$


Third, the optimal system has $\mu_{h}=\bar{\mu}_{2}$. For a system with $\mu_{l}=0$ and $\bar{\mu}_{1}<\mu_{h}<\bar{\mu}_{2}$

$$
\mathbb{E}\left(\hat{v}\left(\mu^{\prime}\right)\right)=\operatorname{Pr}\left(\mu_{h} \mid \pi\right) \hat{v}\left(\mu_{h}\right)=\operatorname{Pr}\left(\mu_{h} \mid \pi\right) \mu_{h}\left(B+s N_{g}\right)=\mu_{0}\left(B+s N_{g}\right)
$$

But a system with $\mu_{h} \geqslant \bar{\mu}_{2}$ achieves a higher expected payoff:

$$
\begin{aligned}
\mathbb{E}_{\pi\left(\mu_{h}, 0\right)}(\hat{v}(\mu)) & =\operatorname{Pr}\left(\mu_{h} \mid \pi\right) \hat{v}\left(\mu_{h}\right)=\operatorname{Pr}\left(\mu_{h} \mid \pi\right)\left(B+s\left(\mu_{h} N_{g}+s\left(1-\mu_{h}\right) N_{b}\right)\right) \\
& =\operatorname{Pr}\left(\mu_{h} \mid \pi\right) B+s\left(\mu_{0} N_{g}+\left(1-\mu_{0}\right) N_{b}\right) \leqslant \mathbb{E}_{\pi\left(\bar{\mu}_{2}, 0\right)}(\hat{v}(\mu))
\end{aligned}
$$

So the optimal system has $\mu_{h}=\bar{\mu}_{2}$ and $\mu_{l}=0$, with $\lambda_{g}=1$ and $\lambda_{b}=$ $\frac{\mu_{0}}{1-\mu_{0}} \frac{1-\bar{\mu}_{2}}{\bar{\mu}_{2}}$.

\section{A.3 Proof of Corollary 1}

Proof. This corollary has two parts.

1. Note that $\bar{\mu}_{2}=1-\frac{m}{(1-s)\left|N_{b}\right|}$. For a mis-aligned manager, the range of market priors in which an uninformative system is optimal is $[\tilde{\mu}, 1]$, where $\tilde{\mu}=\min \left\{\bar{\mu}, \bar{\mu}_{2}\right\}$. As $m$ decreases, $\bar{\mu}_{2}$ and $\tilde{\mu}$ increase. Thus, the range of informative disclosure expands and the range of uninformative disclosure $[\tilde{\mu}, 1]$ shrinks.

2. Note that a mis-aligned manager chooses $\lambda_{g}=1$ and $\lambda_{b}=\frac{\mu_{0}}{1-\mu_{0}} \frac{1-\bar{\mu}_{2}}{\bar{\mu}_{2}}$. In the range of priors with informative systems, the disclosure is liberal since $1-\lambda_{g}<\lambda_{b}$. As $m$ decreases, $\lambda_{b}$ decreases, and by the definitions of bias and informativeness in Section 2, the system becomes less liberal and more informative. 


\section{A.4 Proof of Corollary 2}

Proof. For a common prior belief $\mu_{0} \in(0, \tilde{\mu})$, the optimal system has $\mu_{l}=0$ and $\mu_{h}=\tilde{\mu}$, and thus $\operatorname{Pr}(h)=\frac{\mu_{0}}{\tilde{\mu}}$. If $\mu_{0} \in(\tilde{\mu}, 1)$, the system is uninformative and the investor approves outright with probability 1 . Thus, the probability of investment

is $\min \left\{\frac{\mu_{0}}{\tilde{\mu}}, 1\right\}$, while the efficient level of investment is $\mu_{0}$ (only good projects are approved, and the probability that a project is good is $\left.\mu_{0}\right)$. The firm over-invests in some bad projects when $\mu_{0}<\tilde{\mu}$ (note $\lambda_{g}=1$ and $\lambda_{b}>0$ ), and also when $\tilde{\mu} \leqslant \mu_{0}<1$ (the system is uninformative and the investor invests with probability 1).

Thus, the level of over-investment is $\min \left\{\frac{\mu_{0}}{\tilde{\mu}}, 1\right\}-\mu_{0}$. As $m$ decreases, $\bar{\mu}_{2}$ weakly increases and over-investment weakly decreases.

\section{A.5 Proof of Proposition 2}

Proof. When two systems give the manager the same expected gross payoff, the manager prefers the least informative system since it is least costly.

1. If $m \geqslant \bar{m}$ : consider any system $\pi$ :

$$
\begin{aligned}
\mathbb{E}(\hat{v}(\mu)) & =\mathbb{E}\left(\mathbf{1}(\mu \geqslant \bar{\mu})\left(B+s\left(\mu N_{g}+(1-\mu) N_{b}\right)\right)\right) \\
& =\mathbb{E}\left(\mathbf{1}(\mu \geqslant \bar{\mu}) \mu\left(B+s N_{g}\right)\right)+\mathbb{E}\left(\mathbf{1}(\mu \geqslant \bar{\mu})(1-\mu)\left(B+s N_{b}\right)\right) \\
& \leqslant \mathbb{E}\left(\mathbf{1}(\mu \geqslant \bar{\mu}) \mu\left(B+s N_{g}\right)\right) \leqslant \mu_{0}\left(B+s N_{g}\right)=v_{F I}\left(\mu_{0}\right)
\end{aligned}
$$

The optimal reporting system is fully revealing.

2. If $m<\bar{m}$ : First note that for any $\mu_{0}$, the maximum expected payoff to the manager has an upper bound. For any system $\pi$,

$$
\begin{aligned}
\mathbb{E}(\hat{v}(\mu)) & =\mathbb{E}\left(\mathbf{1}\left(\mu \geqslant \bar{\mu}_{1}\right) \mu\left(B+s N_{g}\right)+\mathbf{1}\left(\mu>\bar{\mu}_{2}\right)(1-\mu)\left(B+s N_{b}\right)\right) \\
& \leqslant \mathbb{E}\left(\mu\left(B+s N_{g}\right)\right)=\mu_{0}\left(B+s N_{g}\right)
\end{aligned}
$$


(a) If $\mu_{0}>\bar{\mu}_{2}$ : Under the uninformative system, the manager's expected payoff is:

$$
\hat{v}\left(\mu_{0}\right)=\left(B+s\left(\mu_{0} N_{g}+\left(1-\mu_{0}\right) N_{b}\right)\right)
$$

Smaller than $\mu_{0}\left(B+s N_{g}\right)$. But with $\mu_{h}=1$ and $\mu_{l}=\bar{\mu}_{2}$, the manager's expected payoff is:

$$
\begin{aligned}
& \mathbb{E}_{\pi\left(1, \bar{\mu}_{2}\right)}(\hat{v}(\mu)) \\
= & \mathbb{E}_{\pi\left(1, \bar{\mu}_{2}\right)}\left(\mathbf{1}\left(\mu \geqslant \bar{\mu}_{1}\right) \mu\left(B+s N_{g}\right)+\mathbf{1}\left(\mu>\bar{\mu}_{2}\right)(1-\mu)\left(B+s N_{b}\right)\right) \\
= & \mu_{0}\left(B+s N_{g}\right)
\end{aligned}
$$

When $\mu=\bar{\mu}_{2}$, the investor is indifferent between inspecting at cost $m$ and investing without inspection, and I assume that she chooses the wellaligned manager's favored action, i.e., to inspect the project. The optimal disclosure must have $\mu_{h}=1$. Assume $1>\mu_{h} \geqslant \mu_{0}>\bar{\mu}_{2}$,

$$
\begin{aligned}
& \mathbb{E}_{\pi\left(\mu_{h}, \mu_{l}\right)}(\hat{v}(\mu)) \\
= & \mathbb{E}_{\pi\left(\mu_{h}, \mu_{l}\right)}\left(\mathbf{1}\left(\mu \geqslant \bar{\mu}_{1}\right) \mu\left(B+s N_{g}\right)+\mathbf{1}\left(\mu>\bar{\mu}_{2}\right)(1-\mu)\left(B+s N_{b}\right)\right) \\
< & \mathbb{E}_{\pi\left(\mu_{h}, \mu_{l}\right)}\left(\mathbf{1}\left(\mu \geqslant \bar{\mu}_{1}\right) \mu\left(B+s N_{g}\right)\right) \leqslant \mu_{0}\left(B+s N_{g}\right)
\end{aligned}
$$

Only when $\mu_{h}=1$ and $\mu_{l} \leqslant \bar{\mu}_{2}$, we have $\mathbb{E}_{\pi\left(\mu_{h}, \mu_{l}\right)}(\hat{v}(\mu))=\mu_{0}\left(B+s N_{g}\right)$. Since the manager chooses the least informative system within a class of systems with the same expected payoff, the optimal system has $\mu_{h}=1$ and $\mu_{l}=\bar{\mu}_{2}$. Thus $\lambda_{b}=0$ and $\lambda_{g}=1-\frac{1-\mu_{0}}{\mu_{0}} \frac{\bar{\mu}_{2}}{1-\bar{\mu}_{2}}$.

(b) If $\bar{\mu}_{1} \leqslant \mu_{0}<\bar{\mu}_{2}$ : The optimal system is uninformative. In this case, under the uninformative system, the investor will inspect and the manager's expected payoff is $\hat{v}\left(\mu_{0}\right)=\mu_{0}\left(B+s N_{g}\right)$, which is the highest achievable payoff. Other systems with $\bar{\mu}_{1} \leqslant \mu_{l} \leqslant \mu_{h} \leqslant \bar{\mu}_{2}$ all give the same expected payoff. Since the manager picks the least informative one among the 
class of systems with the same expected payoff, his optimal system is uninformative with $\mu_{h}=\mu_{l}=\mu_{0}$.

(c) If $\mu_{0}<\bar{\mu}_{1}$ : First, the optimal system must have $\mu_{h} \geqslant \bar{\mu}_{1}$. Otherwise, since $\mu_{l} \leqslant \mu_{h}<\bar{\mu}_{1}$, no investment occurs and the manager's payoff is 0 . Second, the optimal system must have $\mu_{l}=0$. Otherwise, if $\mu_{l}>0$, an alternative system with the same $\mu_{h}$ but $\mu_{l}=0$ will yield a higher expected payoff:

$$
\mathbb{E}_{\pi\left(\mu_{h}, \mu_{l}\right)}(\hat{v}(\mu))=\operatorname{Pr}\left(\mu_{h} \mid \pi\right) \hat{v}\left(\mu_{h}\right)<\operatorname{Pr}\left(\mu_{h} \mid \pi^{\prime}\right) \hat{v}\left(\mu_{h}\right)=\mathbb{E}_{\pi\left(\mu_{h}, 0\right)}(\hat{v}(\mu))
$$

Systems with $\bar{\mu}_{1} \leqslant \mu_{h} \leqslant \bar{\mu}_{2}$ and $\mu_{l}=0$ achieve the maximum expected payoff.

$$
\mathbb{E}_{\pi\left(\mu_{h} \geqslant \bar{\mu}_{1}, 0\right)}(\hat{v}(\mu))=\mu_{0}\left(B+s N_{g}\right)
$$

The optimal system has $\mu_{l}=0$ and $\mu_{h}=\bar{\mu}_{1}$, since this is the least informative system among the class that obtains the highest payoff. The manager chooses $\lambda_{g}=1$ and $\lambda_{b}=\frac{\mu_{0}}{1-\mu_{0}} \frac{1-\bar{\mu}_{1}}{\bar{\mu}_{1}}$.

\section{A.6 Proof of Corollary 3}

Proof. This corollary has two parts.

1. For a well-aligned manager, when $m<\bar{m}$, as $m$ decreases, $\bar{\mu}_{1}=\frac{m}{(1-s) N_{g}}$ decreases, and $\bar{\mu}_{2}$ increases. Thus, the range of priors over which the manager prefers uninformative systems, $\mathbb{U}=\left[\bar{\mu}_{1}, \bar{\mu}_{2}\right]$, expands. The range of priors over which the manager prefers informative systems, $\mathbb{I}=\left(0, \bar{\mu}_{1}\right) \cup\left(\bar{\mu}_{2}, 1\right)$, shrinks.

2. When $\mu_{0} \in\left(0, \bar{\mu}_{1}\right)$, the optimal system has $\lambda_{g}=1$ and $\lambda_{b}=\frac{\mu_{0}}{1-\mu_{0}} \frac{1-\bar{\mu}_{1}}{\bar{\mu}_{1}}$. The system is liberal since $1-\lambda_{b}<\lambda_{g}$. When $m$ decreases, $\lambda_{b}$ increases; thus $\mu_{h}$ 
decreases and $\mu_{l}$ remains 0 , and the system becomes more liberal and less informative.

3. When $\mu_{0} \in\left(\bar{\mu}_{2}, 1\right)$, the optimal system has $\lambda_{b}=0$ and $\lambda_{g}=1-\frac{1-\mu_{0}}{\mu_{0}} \frac{\bar{\mu}_{2}}{1-\bar{\mu}_{2}}$. The system is conservative since $\lambda_{g}<1-\lambda_{b}$. When $m$ decreases, $\lambda_{g}$ decreases; thus $\mu_{l}$ increases and $\mu_{h}$ remains 1 , and the system becomes less informative and more conservative.

\section{A.7 Proof of Lemma 2}

Proof. First note that once the private information is disclosed and the information asymmetry is resolved, the manager can choose the optimal system the same as in the basic model. If $\mu^{\prime}>\mu^{\prime \prime}>\tilde{\mu}$, managers will choose the uninformative system regardless of their private information.

If $\mu^{\prime \prime}<\mu^{\prime}<\tilde{\mu}$, there is a separating equilibrium in which only the manager with $\mu^{\prime}$ voluntarily discloses his private information. The manager with less favorable private information $\left(\mu^{\prime \prime}\right)$ has no incentive to disclose, since disclosure does not benefit him and is costly. He chooses a system with $\lambda_{g}=1$ and $\lambda_{b}=\frac{\mu^{\prime \prime}}{1-\mu^{\prime \prime}} \frac{1-\tilde{\mu}}{\tilde{\mu}}$. The manager with more favorable private information $\left(\mu^{\prime}\right)$ has an incentive to disclose because he can choose a more liberal system and obtain a higher expected payoff when disclosure results in a higher common belief about the project's profitability; note that with common prior belief $\mu$, the mis-aligned manager's optimal system has $\lambda_{g}=1$ and $\lambda_{b}=\frac{\mu}{1-\mu} \frac{1-\tilde{\mu}}{\tilde{\mu}}$, with $\lambda_{b}$ increasing with $\mu$. The investor's optimal action is to reject outright upon seeing a $l$-signal and to approve outright upon seeing a $h$-signal. Thus 
the manager discloses if the benefit from disclosure exceeds its cost:

$$
\begin{aligned}
d & \leqslant V\left(\mu^{\prime}\right)-V\left(\mu^{\prime \prime}\right) \\
& =\left(1-\mu^{\prime}\right) \frac{1-\tilde{\mu}}{\tilde{\mu}}\left(\frac{\mu^{\prime}}{1-\mu^{\prime}}-\frac{\mu^{\prime \prime}}{1-\mu^{\prime \prime}}\right)\left(B+s N_{b}\right)
\end{aligned}
$$

Similarly, if $\mu^{\prime \prime}<\tilde{\mu}<\mu^{\prime}$, the manager with $\mu^{\prime}$ has incentive to disclose voluntarily if the cost is sufficiently low: $d \leqslant\left(1-\mu^{\prime}\right)\left(B+s N_{b}\right)\left(1-\frac{\mu^{\prime \prime}}{1-\mu^{\prime \prime}} \frac{1-\tilde{\mu}}{\tilde{\mu}}\right)$.

\section{A.8 Proof of Lemma 3}

Proof. When the manager cannot disclose his private information truthfully at a sufficiently low cost, the mis-aligned manager's optimal system remains the same as in the basic model.

First I show that a separating equilibrium does not exist. Suppose a separating equilibrium exists, under which the manager with private information $\mu^{\prime}$ chooses System $\pi^{\prime}$, and the manager with $\mu^{\prime \prime}$ chooses System $\pi^{\prime \prime}$, and $\pi^{\prime} \neq \pi^{\prime \prime}$. The investor learns the manager's private information by observing their choices of system. Also, the manager with $\mu^{\prime}$ prefers System $\pi^{\prime}$ to $\pi^{\prime \prime}$, while the manager with $\mu^{\prime \prime}$ prefers System $\pi^{\prime \prime}$ to $\pi^{\prime}$. Since a l-signal leads to outright rejection and zero payoff to the manager, the Incentive Compatibility Constraints can be written as follows:

$$
\begin{aligned}
\mu^{\prime} \lambda_{g}^{\prime}+\left(1-\mu^{\prime}\right) \lambda_{b}^{\prime} & \geqslant \mu^{\prime} \lambda_{g}^{\prime \prime}+\left(1-\mu^{\prime}\right) \lambda_{b}^{\prime \prime} \\
\mu^{\prime \prime} \lambda_{g}^{\prime \prime}+\left(1-\mu^{\prime \prime}\right) \lambda_{b}^{\prime \prime} & \geqslant \mu^{\prime \prime} \lambda_{g}^{\prime}+\left(1-\mu^{\prime \prime}\right) \lambda_{b}^{\prime}
\end{aligned}
$$

which implies

$$
\mu^{\prime}\left(\lambda_{g}^{\prime}-\lambda_{g}^{\prime \prime}\right) \geqslant\left(1-\mu^{\prime}\right)\left(\lambda_{b}^{\prime \prime}-\lambda_{b}^{\prime}\right) \geqslant\left(1-\mu^{\prime}\right) \frac{\mu^{\prime \prime}}{\left(1-\mu^{\prime \prime}\right)}\left(\lambda_{g}^{\prime}-\lambda_{g}^{\prime \prime}\right)
$$

Since $\mu^{\prime}>\mu^{\prime \prime}$, it must be $1 \geqslant \lambda_{g}^{\prime}>\lambda_{g}^{\prime \prime}$. However, the manager with the less favorable private information $\mu^{\prime \prime}$ can always achieve a higher expected payoff by deviating to 
the system with $\lambda_{g}^{\prime \prime}=1$ and $\lambda_{b}^{\prime \prime}=\frac{\mu^{\prime \prime}}{1-\mu^{\prime \prime}} \frac{1-\tilde{\mu}}{\tilde{\mu}}$, which contradicts $1>\lambda_{g}^{\prime \prime}$. Thus, there is no separating equilibrium.

Next note that a pooling equilibrium exists, and the optimal system has $\lambda_{g}=1$ and $\lambda_{b}=\frac{\mu_{0}}{1-\mu_{0}} \frac{1-\tilde{\mu}}{\tilde{\mu}}$. Under this system, the investor approves outright upon seeing a $h$-signal and rejects outright upon seeing a $l$-signal. If a manager deviates, the investor has prior belief $\mu_{0}$ and updates her posterior belief according to the Bayes' Rule. This system gives the manager the highest expected payoff regardless of his private information.

\section{A.9 Proof of Lemma 4}

Proof. The proof is similar to the proof of the Revelation Principle. Consider the optimal reporting system when $\delta>0$. In equilibrium, the manager may or may not manipulate. Suppose, in equilibrium, the investor's posteriors upon receiving the disclosed signals are $\mu_{h^{\prime}}$ and $\mu_{l^{\prime}}$. Since the investor interprets the signals rationally, $\mathbb{E}\left(\mu_{y^{\prime}}\right)=\mu_{0}$; i.e., the post-manipulation posterior must satisfy the Bayesplausibility constraint. The manager's expected payoff $\mathbb{E}\left(r_{y}\right)=\mathbb{E}\left(\hat{v}\left(\mu_{y}^{\prime}\right)\right)-\Phi$, in which $\mathbb{E}\left(\hat{v}\left(\mu_{y}^{\prime}\right)\right)$ is determined by this distribution of the investor's posterior, and $\Phi \geqslant 0$ is the expected cost from manipulation. For any distribution of $\mu_{h^{\prime}}, \mu_{l^{\prime}}$, the manager can always find a system $\pi$ under the no-manipulation case that generates the same distribution of posteriors $\left(\mu_{h}=\mu_{h^{\prime}}\right.$ and $\left.\mu_{l}=\mu_{l^{\prime}}\right)$. In the no-manipulation case, this system yields the same expected gross payoff, but will not incur the cost of manipulation. Thus, the manager is ex ante better off in the no-manipulation case. 


\section{A.10 Proof of Lemma 5}

Proof. The manager's maximum payoff in the manipulation case will not exceed his payoff in the no-manipulation case. For a well-aligned manager, if his optimal system in the no-manipulation case yields the same expected payoff as in the manipulation case, this system must be optimal in the manipulation case as well. This is indeed the case. Under the optimal system from the no-manipulation case, the well-aligned manager has no incentive to manipulate, since the investor will always take the manager's favored action. Manipulation is then not only costly, but may also lead the investor to choose a less desirable action. Thus, allowing the well-aligned manager to manipulate the disclosed signal will not change his reporting system.

\section{A.11 Lemma A1 and its proof}

Lemma A1. When manipulation is allowed, a mis-aligned manager never manipulates when he privately observes a h-signal, i.e., $\phi_{h}=0$.

Lemma A1 shows that the manager with a $h$-signal never manipulates. In the following discussion, I simplify the notation $\mu_{y^{\prime}}\left(\phi_{h}, \phi_{l}\right)$ to $\mu_{y^{\prime}}\left(\phi_{l}\right)$.

Proof. Suppose the reporting system is in place and the original signals $h(l)$ lead to posterior $\mu_{h}\left(\mu_{l}\right)$. The requirement $\lambda_{g} \geqslant \lambda_{b}$ ensures $\mu_{h} \geqslant \mu_{l}$. The proof has two steps. First, I show that at most one type of manager will manipulate; second, I show that the manager with a $h$-signal never manipulates.

1. Suppose managers with both $h$-signal and $l$-signal manipulate. Then

$$
\begin{aligned}
& (1-\delta) \hat{v}\left(\mu_{h^{\prime}}\right)+\delta \hat{v}\left(\mu_{l^{\prime}}\right)-F \geqslant \hat{v}\left(\mu_{h^{\prime}}\right) \\
& (1-\delta) \hat{v}\left(\mu_{l^{\prime}}\right)+\delta \hat{v}\left(\mu_{h^{\prime}}\right)-F \geqslant \hat{v}\left(\mu_{l^{\prime}}\right)
\end{aligned}
$$

This implies $-F \geqslant \delta\left(\hat{v}\left(\mu_{h^{\prime}}\right)-\hat{v}\left(\mu_{l^{\prime}}\right)\right) \geqslant F$, which is a contradiction. Thus, at most one type manipulates. 
2. Suppose the manager with a $h$-signal manipulates. Then $\hat{v}\left(\mu_{l^{\prime}}\right)>\hat{v}\left(\mu_{h^{\prime}}\right)$. However, since $\mu_{h} \geqslant \mu_{l}$, we have $\mu_{h^{\prime}}\left(\phi_{h}, 0\right)=\mu_{h}>\mu_{l^{\prime}}\left(\phi_{h}, 0\right)$, and $\hat{v}\left(\mu_{h^{\prime}}\left(\phi_{h}, 0\right)\right)>$ $\hat{v}\left(\mu_{l^{\prime}}\left(\phi_{h}, 0\right)\right)$, which is a contradiction. Thus, a $h$-signal manager will not manipulate, and $\phi_{h}=0$ in equilibrium. Only managers with a $l$-signal may manipulate $\left(\phi_{l} \geqslant 0\right)$.

\section{A.12 Lemma A2 and its proof}

Lemma A2. Assume $\lambda_{g}=1$. Upon observing the original output $y$, the mis-aligned manager's manipulation decision and the investor's inspection decision are as follows:

1. If $\lambda_{b}>\frac{1-\tilde{\mu}}{\tilde{\mu}} \frac{\mu_{0}}{1-\mu_{0}}$ : The l-signal manager does not manipulate $\left(\phi_{l}=0\right)$. The investor inspects if $\mu_{y^{\prime}}\left(\phi_{l}=0\right) \geqslant \bar{\mu}_{1}$, and invests only if inspection reveals a good state.

2. If $\frac{1}{1-\delta}\left(\frac{1-\tilde{\mu}}{\tilde{\mu}} \frac{\mu_{0}}{1-\mu_{0}}-\delta\right)<\lambda_{b}<\frac{1-\tilde{\mu}}{\tilde{\mu}} \frac{\mu_{0}}{1-\mu_{0}}$ : The l-signal manager manipulates with probability $\phi_{l}=\frac{1}{\delta}\left(\frac{\mu_{0}(1-\tilde{\mu}) \lambda_{g}}{\left(1-\mu_{0}\right) \tilde{\mu}}-\lambda_{b}\right)$. The investor invests without inspection upon seeing the $h^{\prime}$-signal with probability $\chi_{h}=\frac{F}{\delta\left(B+s N_{b}\right)}$.

3. If $\lambda_{b}<\frac{1}{1-\delta}\left(\frac{1-\tilde{\mu}}{\tilde{\mu}} \frac{\mu_{0}}{1-\mu_{0}}-\delta\right)$ : The l-signal manager manipulates with probability $\phi_{l}=1$. The investor invests without inspection upon seeing the $h^{\prime}$-signal with probability $\chi_{h^{\prime}}=1$.

Lemma A2 shows that the $l$-signal manager manipulates only if manipulation increases the probability of approval without inspection, since he knows that his project is bad for sure $\left(\mu_{l^{\prime}}\left(\phi_{l}\right)=0\right)$, which will be revealed in an inspection. Furthermore, a more liberal system leads to less manipulation. That is, $\phi_{l}$ weakly decreases in $\lambda_{b}$. This is intuitive, as a higher $\lambda_{b}$ makes the $h^{\prime}$-signal less indicative of a good state 
and the investor less willing to invest. The lower payoff to a $h^{\prime}$-signal leads to less manipulation.

Proof. There are three cases.

1. If $\lambda_{b}>\frac{1-\tilde{\mu}}{\tilde{\mu}} \frac{\mu_{0}}{1-\mu_{0}}$ : In this case, the original signal yields posteriors $\mu_{h}<\tilde{\mu}$ and $\mu_{l}=0$. The disclosed signal has $\mu_{h^{\prime}}\left(\phi_{l}\right)<\tilde{\mu}$, regardless of $\phi_{l}$, and a $h^{\prime}$-signal leads to inspection if $\mu_{h^{\prime}}\left(\phi_{l}\right)>\bar{\mu}_{1}$, or outright rejection if $\mu_{h^{\prime}}\left(\phi_{l}\right)<\bar{\mu}_{1}$. In either case, the l-signal manager has no incentive to manipulate, since he knows for sure that his project is bad and will be revealed by the inspection. Thus, in equilibrium $\phi_{l}=0$.

2. If $\frac{1}{1-\delta}\left(\frac{1-\tilde{\mu}}{\tilde{\mu}} \frac{\mu_{0}}{1-\mu_{0}}-\delta\right)<\lambda_{b}<\frac{1-\tilde{\mu}}{\tilde{\mu}} \frac{\mu_{0}}{1-\mu_{0}}$ : In this case, the original signal gives posteriors $\mu_{h}>\tilde{\mu}$ and $\mu_{l}=0$, but the disclosed signal has $\mu_{h^{\prime}}(1)<\tilde{\mu}<\mu_{h^{\prime}}(0)$. It can not constitute an equilibrium if the l-signal manager employs a pure strategy, i.e., if he manipulates for sure or if he never manipulates. If he manipulates for sure, the investor's posterior $\mu_{h^{\prime}}(1)<\tilde{\mu}$ is too low to justify outright approval, and hence manipulation does not pay off. On the other hand, if he never manipulates, then $\mu_{h^{\prime}}(0)>\tilde{\mu}$, and the investor approves upon seeing the $h^{\prime}$ signal with certainty, which leads the manager to strictly prefer manipulation. Thus, in equilibrium, the $l$-signal manager manipulates with a positive probability $\phi_{l}$ to make the investor indifferent between approval and inspection, and the investor approves upon seeing the $h^{\prime}$-signal with probability $\chi_{h^{\prime}}$ to make the $l$-signal manager indifferent between manipulation or not. This requires that $\mu_{h^{\prime}}\left(\phi_{l}\right)=\tilde{\mu}$, and $\chi_{h^{\prime}}\left(B+s N_{b}\right)-F=0$, i.e., $\phi_{l}=\frac{1}{\delta}\left(\frac{\mu_{0}(1-\tilde{\mu}) \lambda_{g}}{\left(1-\mu_{0}\right) \tilde{\mu}}-\lambda_{b}\right)$ and $\chi_{h^{\prime}}=\frac{F}{\delta\left(B+s N_{b}\right)}$.

3. If $\lambda_{b}<\frac{1}{1-\delta}\left(\frac{1-\tilde{\mu}}{\tilde{\mu}} \frac{\mu_{0}}{1-\mu_{0}}-\delta\right)$ : In this case, the original signal gives posteriors 
$\mu_{h}>\tilde{\mu}$ and $\mu_{l}=0$. The disclosed signal has $\mu_{h^{\prime}}(1)>\tilde{\mu}$. That is, even if the $l$-signal manager manipulates for sure, the investor is still willing to approve without inspection upon seeing the $h^{\prime}$-signal. For a $l$-signal manager, the expected payoff from manipulation is $\delta\left(B+s N_{b}\right)$, greater than the cost $F$. Thus, he manipulates with certainty. $\chi_{h^{\prime}}=1$ and $\phi_{l}=1$ constitutes an equilibrium.

\section{A.13 Lemma A3 and its proof}

Lemma A3. When $m \geqslant \bar{m}$, depending on the investor's response to a $h^{\prime}$-signal, the systems that give the mis-aligned manager a positive expected payoff can be classified into two exhaustive and mutually exclusive types. Denote $r_{y}=\mathbb{E}\left(\hat{v}\left(\mu_{y^{\prime}}\left(\phi_{h}, \phi_{l}\right)\right) \mid y\right)$, the manager's expected payoff conditional on observing an original signal $y$. The types and the optimal system within each type are identified as follows.

1. Systems that induce the investor to approve outright with probability $\chi_{h^{\prime}}<$ 1 upon receiving the $h^{\prime}$-signal. Among this class, the optimal system is $\pi_{1}$ with $\lambda_{g}=1$ and $\lambda_{b}=\frac{1-\bar{\mu}}{\bar{\mu}} \frac{\mu_{0}}{1-\mu_{0}}$. Under $\pi_{1}$, upon seeing a $h^{\prime}$-signal, the investor approves outright with probability $\chi_{h^{\prime}}=\frac{F}{\delta\left(B+s N_{b}\right)}$, and rejects outright with probability $1-\chi_{h^{\prime}}$. The manager's expected payoff is $\mathbb{E}_{\pi_{1}}\left(r_{y}\left(\mu_{0}\right)\right)=$ $\frac{F}{\delta\left(B+s N_{b}\right)}\left(\mu_{0}\left(B+s N_{g}\right)+\mu_{0} \frac{1-\bar{\mu}}{\bar{\mu}}\left(B+s N_{b}\right)\right)$.

2. Systems that induce the investor to approve outright upon receiving the $h^{\prime}$ signal. Among this class, the optimal system is $\pi_{2}$ with $\lambda_{g}=1$ and $\lambda_{b}=$ $\frac{1}{1-\delta}\left(\frac{1-\bar{\mu}}{\bar{\mu}} \frac{\mu_{0}}{1-\mu_{0}}-\delta\right)$. Under $\pi_{2}$, the investor approves outright upon seeing a $h^{\prime}-$ signal with probability $\chi_{h^{\prime}}=1$, and the l-signal manager manipulates with probability $\phi_{l}=1$. The manager's expected payoff is $\mathbb{E}_{\pi_{2}}\left(r_{y}\left(\mu_{0}\right)\right)=\mu_{0}\left(B+s N_{g}\right)+$ 


$$
\mu_{0} \frac{1-\bar{\mu}}{\bar{\mu}}\left(B+s N_{b}\right)-\left(1-\mu_{0}\right)\left(1-\frac{1}{1-\delta}\left(\frac{1-\bar{\mu}}{\bar{\mu}} \frac{\mu_{0}}{1-\mu_{0}}-\delta\right)\right) F
$$

Lemma A3 introduces the reporting systems that are optimal within a given type of system, and are thus potential candidates for the optimal system.

Proof. The idea in the proof of Lemma A3 is that, when $m \geqslant \bar{m}$, for any reporting system $\pi$ with $\lambda_{g}<1$, there exists a reporting system with $\lambda_{g}=1$ that gives the mis-aligned manager a weakly higher payoff in expectation. Suppose, in equilibrium, the $l$-signal manager manipulates with probability $\phi_{l}$. I first classify all reporting systems into two types and identify the optimal system within each type.

1. Systems that induce the investor to approve outright with probability $\left(0<\chi_{h^{\prime}}<1\right)$ : Since in equilibrium the investor uses a mixed strategy, she must be indifferent between outright approval and outright rejection, which is possible only when $\mu_{h^{\prime}}\left(\phi_{l}\right)=\bar{\mu}$. In addition, to make the manager indifferent between manipulating or not, $\hat{v}\left(\mu_{l^{\prime}}\right)=0$ and $\chi_{h^{\prime}}=\frac{F}{\delta\left(B+s\left(\mu_{l} N_{g}+\left(1-\mu_{l}\right) N_{b}\right)\right)}$. Thus $\chi_{h^{\prime}}$ decreases in $\mu_{l}$. Since the manager's ex-ante expected payoff is $\operatorname{Pr}\left(\mu_{h^{\prime}} \mid \pi\right) \chi_{h^{\prime}}\left(B+s\left(\bar{\mu} N_{g}+(1-\bar{\mu}) N_{b}\right)\right)$, System $\pi_{1}$ with $\mu_{h}=\bar{\mu}$ and $\mu_{l}=0$ is optimal within this class, which maximizes $\chi_{h^{\prime}} \operatorname{Pr}\left(\mu_{h^{\prime}} \mid \pi\right)$. Under System $\pi_{1}$, in equilibrium, managers with either $h$ or l-signal do not manipulate, and the investor's posterior is either $\mu_{h^{\prime}}=\bar{\mu}$ or $\mu_{l^{\prime}}=0$. The investor approves outright upon seeing a $h^{\prime}$-signal with probability $\chi_{h^{\prime}}=\frac{F}{\delta\left(B+s N_{b}\right)}$. Under System $\pi_{1}$, the manager's expected payoff is:

$$
\begin{aligned}
\mathbb{E}_{\pi_{1}}\left(r_{y} \mid m \geqslant \bar{m}\right) & =\mathbb{E}_{\pi_{1}}\left(\hat{v}\left(\mu_{y^{\prime}}\right)\right)-\Phi_{\pi_{1}} \\
& =\chi_{h^{\prime}, 1} \operatorname{Pr}\left(h^{\prime}\right) v\left(\mu_{h^{\prime}}, a=A\right) \\
& =\frac{F}{\delta\left(B+s N_{b}\right)}\left[\mu_{0}\left(B+s N_{g}\right)+\mu_{0} \frac{1-\bar{\mu}}{\bar{\mu}}\left(B+s N_{b}\right)\right]
\end{aligned}
$$

2. Systems that induce outright approval $\left(\chi_{h^{\prime}}=1\right)$ : In this type of system, in equilibrium, $\mu_{h^{\prime}}\left(\phi_{h}, \phi_{l}\right) \geqslant \bar{\mu}$ and $\chi_{h^{\prime}}=1$. The investor approves outright upon 
receiving a $h^{\prime}$-signal. I show that System $\pi_{2}\left(\lambda_{g}=1, \lambda_{b}=\frac{1}{1-\delta}\left(\frac{1-\bar{\mu}}{\bar{\mu}} \frac{\mu_{0}}{1-\mu_{0}}-\delta\right)\right)$ achieves the highest expected payoff among this type of system. Under System $\pi_{2}$, the original signal has $\mu_{h}>\bar{\mu}$ and $\mu_{l}=0$. A $l$-signal manager manipulates with certainty $\left(\phi_{l}=1\right)$, and the investor's posteriors are $\mu_{h^{\prime}}=\bar{\mu}$ and $\mu_{l^{\prime}}=0$. As such, the $h^{\prime}$-signal is sufficiently convincing that upon seeing a $h^{\prime}$-signal the investor approves outright. For any system $\pi$ in this class, I show that $\mathbb{E}_{\pi}(\hat{v}(\mu)) \leqslant \mathbb{E}_{\pi_{2}}(\hat{v}(\mu))$ by similar proof of Proposition 1. Furthermore, note that the expected manipulation cost is

$$
\Phi_{\pi}=F\left(\left(1-\mu_{0}\right)\left(1-\lambda_{b}\right)+\mu_{0}\left(1-\lambda_{g}\right)\right)
$$

Thus, System $\pi_{2}$ gives the lowest manipulation cost since it has the largest $\lambda_{g}$, $\lambda_{b}$ within this type. Thus, $\pi_{2}$ achieves the highest expected payoff within this class:

$$
\mathbb{E}_{\pi}\left(r_{y}\right)=\mathbb{E}_{\pi}\left(\hat{v}_{y^{\prime}}\right)-\Phi_{\pi} \leqslant \mathbb{E}_{\pi_{2}}(\hat{v}(\mu))-\Phi_{\pi_{2}}
$$

And the mis-aligned manager's expected payoff under System $\pi_{2}$ is:

$$
\begin{aligned}
& \mathbb{E}_{\pi_{2}}\left(r_{y}\right) \\
= & \mathbb{E}_{\pi_{2}}\left(\hat{v}\left(\mu_{y^{\prime}}\right)\right)-\Phi_{\pi_{2}}=\operatorname{Pr}\left(h^{\prime}\right) v\left(\mu_{h^{\prime}}, a=A\right)-\left(1-\mu_{0}\right)\left(1-\lambda_{b, 2}\right) F \\
= & \mu_{0}\left(B+s N_{g}\right)+\mu_{0} \frac{1-\bar{\mu}}{\bar{\mu}}\left(B+s N_{b}\right)-\left(1-\mu_{0}\right)\left(1-\frac{1}{1-\delta}\left(\frac{1-\bar{\mu}}{\bar{\mu}} \frac{\mu_{0}}{1-\mu_{0}}-\delta\right)\right) F
\end{aligned}
$$

To summarize, when $m \geqslant \bar{m}$, the optimal system is either System $\pi_{1}$ or $\pi_{2}$, and always has $\lambda_{g}=1$.

\section{A.14 Proof of Proposition 3}

Proof. To prove Proposition 3, I first prove Lemmas A1, A2, and A3 (see above). With these lemmas, I next prove Proposition 3. When $m>\bar{m}$ and $\mu_{0}<\bar{\mu}$, 
1. If $\delta>\frac{\mu_{0}}{1-\mu_{0}} \frac{1-\bar{\mu}}{\bar{\mu}}$ : From Lemma A3, the optimal system is either System $\pi_{1}$ or System $\pi_{2}$. When $\delta>\frac{\mu_{0}}{1-\mu_{0}} \frac{1-\bar{\mu}}{\bar{\mu}}$, System $\pi_{2}$ gives a zero expected payoff since $\mu_{h^{\prime}}\left(\phi_{l}\right)<\bar{\mu}$ : The scope of manipulation $\delta$ is so large that a $h^{\prime}$-signal can not convince the investor to provide outright approval. Thus, the optimal system must be $\pi_{1}$.

2. If $\delta<\frac{\mu_{0}}{1-\mu_{0}} \frac{1-\bar{\mu}}{\bar{\mu}}$ : In this case, both $\pi_{1}$ and $\pi_{2}$ are potentially optimal. Since $\mathbb{E}_{\pi_{1}}\left(r_{y}\right)$ increases in $F$ and $\mathbb{E}_{\pi_{2}}\left(r_{y}\right)$ decreases in $F$, the optimality of the system depends on $F$. Denote $\bar{f}_{1}$ the cutoff $F$ above which the mis-aligned manager prefers System $\pi_{1}$ to System $\pi_{2}: \bar{f}_{1}=\frac{\left(B+s N_{g}\right)+\frac{1-\bar{\mu}}{\bar{\mu}}\left(B+s N_{b}\right)}{\frac{1-\mu_{0}}{\mu_{0}}\left(1-\tilde{\lambda}_{b}\right)+\frac{1}{\delta\left(B+s N_{b}\right)}\left(\left(B+s N_{g}\right)+\frac{1-\bar{\mu}}{\bar{\mu}}\left(B+s N_{b}\right)\right)}$, where $\tilde{\lambda}_{b}=\frac{1}{1-\delta}\left(\frac{1-\tilde{\mu}}{\tilde{\mu}} \frac{\mu_{0}}{1-\mu_{0}}-\delta\right)$ is the $\lambda_{b}$ in System $\pi_{2}$.

(a) If $F \leqslant \bar{f}_{1}$ : The optimal system is $\pi_{2}$ with $\lambda_{g}=1, \lambda_{b}=\frac{1}{1-\delta}\left(\frac{1-\bar{\mu}}{\bar{\mu}} \frac{\mu_{0}}{1-\mu_{0}}-\delta\right)$. In equilibrium, the $l$-signal manager manipulates with probability $\phi_{l}=1$. Despite the manipulation, the investor's posterior $\mu_{h^{\prime}}\left(\phi_{l}=1\right)=\bar{\mu}$, and she approves outright with certainty upon seeing a $h^{\prime}$-signal.

(b) If $F>\bar{f}_{1}$ : The optimal system is $\pi_{1}$ with $\lambda_{g}=1, \lambda_{b}=\frac{\mu_{0}}{1-\mu_{0}} \frac{1-\bar{\mu}}{\bar{\mu}}$. In equilibrium, the $l$-signal manager manipulates with probability $\phi_{l}=0$, and the investor's posterior has $\mu_{h^{\prime}}\left(\phi_{l}=1\right)=\bar{\mu}$. However, the investor approves upon seeing a $h^{\prime}$-signal with probability $\chi_{h^{\prime}}=\frac{F}{\delta\left(B+s N_{b}\right)}$.

\section{A.15 Lemma A4 and its proof}

Lemma A4. When $m<\bar{m}$ and $\mu_{0}<\bar{\mu}_{1}$, depending on the investor's response to a $h^{\prime}$-signal, the systems with a positive expected payoff to the mis-aligned manager can 
be classified into three exhaustive and mutually exclusive types. The types and the optimal system within each type are identified as follows.

1. Systems that induce the investor to approve without inspection with probability $\chi_{h^{\prime}}<1$ upon receiving the $h^{\prime}$-signal. Among this class, the optimal system is $\pi_{1}$ with $\lambda_{g}=1$ and $\lambda_{b}=\frac{1-\bar{\mu}_{2}}{\bar{\mu}_{2}} \frac{\mu_{0}}{1-\mu_{0}}$. Under $\pi_{1}$, upon seeing a $h^{\prime}$-signal, the investor approves outright with probability $\chi_{h^{\prime}}=\frac{F}{\delta\left(B+s N_{b}\right)}$, and inspects with probability $1-\chi_{h^{\prime}}$. The manager's expected payoff is $\mathbb{E}_{\pi_{1}}\left(r_{y}\left(\mu_{0}\right)\right)=$ $\mu_{0}\left(B+s N_{g}\right)+\mu_{0} \frac{1-\bar{\mu}_{2}}{\bar{\mu}_{2}} \frac{F}{\delta}$.

2. Systems that induce the investor to approve outright upon receiving the $h^{\prime}$ signal. Among this class, the optimal system is $\pi_{2}$ with $\lambda_{g}=1$ and $\lambda_{b}=$ $\frac{1}{1-\delta}\left(\frac{1-\bar{\mu}_{2}}{\bar{\mu}_{2}} \frac{\mu_{0}}{1-\mu_{0}}-\delta\right)$. Under $\pi_{2}$, the investor approves outright upon seeing a $h^{\prime}$-signal with probability $\chi_{h^{\prime}}=1$, and the l-signal manager manipulates with probability $\phi_{l}=1$. The manager's expected payoff is $\mathbb{E}_{\pi_{2}}\left(r_{y}\left(\mu_{0}\right)\right)=$ $\mu_{0}\left(B+s N_{g}\right)+\mu_{0} \frac{1-\bar{\mu}_{2}}{\bar{\mu}_{2}}\left(B+s N_{b}\right)-\left(1-\mu_{0}\right)\left(1-\frac{1}{1-\delta}\left(\frac{1-\bar{\mu}_{2}}{\bar{\mu}_{2}} \frac{\mu_{0}}{1-\mu_{0}}-\delta\right)\right) F$.

3. Systems that induce inspection when the investor receives a $h^{\prime}$-signal, $\bar{\mu}_{1}<$ $\mu_{h^{\prime}}\left(\phi_{l}\right)<\bar{\mu}_{2}$. Among this class, the optimal system is $\pi_{4}$ with $\lambda_{g}=1$ and $\lambda_{b}=$ $\frac{1-\bar{\mu}_{1}}{\bar{\mu}_{1}} \frac{\mu_{0}}{1-\mu_{0}}$. Under System $\pi_{4}$, the manager's expected payoff is $\mathbb{E}_{\pi_{4}}\left(r_{y}\left(\mu_{0}\right)\right)=$ $\mu_{0}\left(B+s N_{g}\right)$.

Proof. The idea in the proof of Lemma A4 is that, when $m<\bar{m}$ and $\mu_{0}<\bar{\mu}_{1}$, for any reporting system $\pi$ with $\lambda_{g}<1$, there exists a reporting system with $\lambda_{g}=1$ that provides the mis-aligned manager with a weakly higher expected payoff. Suppose, in equilibrium, the $l$-signal manager manipulates with probability $\phi_{l}$. I first classify all reporting systems into three types and identify the optimal system within each type. 
1. Systems that induce the investor to approve outright with probability $(0<$ $\left.\chi_{h^{\prime}}<1\right)$ : Since in equilibrium the investor uses a mixed strategy, she must be indifferent between outright approval and another action, which is possible only when $\mu_{h^{\prime}}\left(\phi_{h}, \phi_{l}\right)=\bar{\mu}_{2}$. I show that the system $\pi_{1}\left(\lambda_{g}=1, \lambda_{b}=\frac{1-\bar{\mu}_{2}}{\bar{\mu}_{2}} \frac{\mu_{0}}{1-\mu_{0}}\right)$ achieves the highest payoff among this class of systems. Under System $\pi_{1}, \mu_{h}=\bar{\mu}_{2}$ and $\mu_{l}=0$. In equilibrium, managers with either a $h$ - or $l$-signal do not manipulate, and the investor's posterior is either $\mu_{h^{\prime}}=\bar{\mu}_{2}$ or $\mu_{l^{\prime}}=0$. She approves outright upon seeing a $h^{\prime}$-signal with probability $\chi_{h^{\prime}}=\frac{F}{\delta\left(B+s N_{b}\right)}$. The reduced approval rate ensures that the manager has no incentive to manipulate.

$$
\mathbb{E}_{\pi}\left(r_{y}\right)=\mathbb{E}_{\pi}\left(\tilde{v}_{y^{\prime}}\right)-\Phi_{\pi} \leqslant \mathbb{E}_{\pi_{1}}(\hat{v}(\mu))-\Phi_{\pi_{1}}
$$

where $\Phi_{\pi}$ is the expected manipulation cost under system $\pi$. This is easy to see that $\Phi_{\pi} \geqslant \Phi_{\pi_{1}}=0$; next I show $\mathbb{E}_{\pi_{1}}(\hat{v}(\mu)) \geqslant \mathbb{E}_{\pi}(\hat{v}(\mu))$. Note that

$$
\begin{aligned}
\delta \hat{v}\left(\mu_{l^{\prime}}\right) & =\delta\left(\chi_{h^{\prime}}\left(B+s\left(\mu_{l} N_{g}+\left(1-\mu_{l}\right) N_{b}\right)\right)+\left(1-\chi_{h^{\prime}}\right) \mu_{l}\left(B+s N_{g}\right)\right)-F \\
\chi_{h^{\prime}} & =\frac{\delta\left(\hat{v}\left(\mu_{l^{\prime}}\right)-\mu_{l}\left(B+s N_{g}\right)\right)+F}{\delta\left(1-\mu_{l}\right)\left(B+s N_{b}\right)}
\end{aligned}
$$

Since $\mu_{l}<\mu_{0}<\bar{\mu}_{1}$, the investor is indifferent between outright approval and rejection; thus $\hat{v}\left(\mu_{l^{\prime}}\right)=0$ and $\chi_{h^{\prime}}=\frac{F-\mu_{l} \delta\left(B+s N_{g}\right)}{\delta\left(1-\mu_{l}\right)\left(B+s N_{b}\right)} . \chi_{h^{\prime}}$ decreases in $\mu_{l}$. As a result, System $\pi_{1}$ with $\mu_{h}=\bar{\mu}_{2}$ and $\mu_{l}=0$ is optimal within this type, since it maximizes $\chi_{h^{\prime}}$ and $\operatorname{Pr}\left(h^{\prime}\right)$. The manager's expected payoff is:

$$
\begin{aligned}
\mathbb{E}_{\pi_{1}}\left(r_{y}\right) & =\chi_{h^{\prime}, 1} \operatorname{Pr}\left(h^{\prime}\right) v\left(\mu_{h^{\prime}}, a=A\right)+\left(1-\chi_{h^{\prime}, 1}\right) \operatorname{Pr}\left(h^{\prime}\right) v\left(\mu_{h^{\prime}}, a=I\right) \\
& =\mu_{0}\left(B+s N_{g}\right)+\frac{F}{\delta} \frac{\mu_{0}}{\bar{\mu}_{2}}\left(1-\bar{\mu}_{2}\right)
\end{aligned}
$$

2. Systems that induce the investor to approve outright $\left(\chi_{h^{\prime}}=1\right)$ : Under this type of systems, in equilibrium, $\mu_{h^{\prime}}\left(\phi_{h}, \phi_{l}\right) \geqslant \bar{\mu}_{2}$ and $\chi_{h^{\prime}}=1$. The investor invests outright upon receiving a $h^{\prime}$-signal. I show that System $\pi_{2}\left(\lambda_{g}=1, \lambda_{b}=\frac{1}{1-\delta}\left(\frac{1-\bar{\mu}_{2}}{\bar{\mu}_{2}} \frac{\mu_{0}}{1-\mu_{0}}-\delta\right)\right)$ 
achieves the highest payoff among this class of systems. Under System $\pi_{2}$, the original signal has $\mu_{h}>\bar{\mu}_{2}$ and $\mu_{l}=0$. In equilibrium, a $l$-signal manager manipulates with certainty $\left(\phi_{l}=1\right)$, and the investor's posteriors are $\mu_{h^{\prime}}=\bar{\mu}_{2}$ and $\mu_{l^{\prime}}=0$. As such, the $h^{\prime}$-signal is sufficiently convincing that the investor approves outright upon seeing a $h^{\prime}$-signal.

$$
\mathbb{E}_{\pi}\left(r_{y}\right)=\mathbb{E}_{\pi}\left(\hat{v}_{y^{\prime}}\right)-\Phi_{\pi} \leqslant \mathbb{E}_{\pi_{2}}(\hat{v}(\mu))-\Phi_{\pi_{2}}
$$

For any system in this class, with similar proof to that of Proposition 1, I show that $\mathbb{E}_{\pi}(\hat{v}(\mu)) \leqslant \mathbb{E}_{\pi_{2}}(\hat{v}(\mu))$, and $\Phi_{\pi} \leqslant \Phi_{\pi_{2}}$, since the expected manipulation cost is $F\left(\left(1-\mu_{0}\right)\left(1-\lambda_{b}\right)+\mu_{0}\left(1-\lambda_{g}\right)\right)$, and System $\pi_{2}$ has $\lambda_{g}=1, \lambda_{b}=\frac{1}{1-\delta}\left(\frac{1-\bar{\mu}_{2}}{\bar{\mu}_{2}} \frac{\mu_{0}}{1-\mu_{0}}-\delta\right)$. Thus, $\pi_{2}$ achieves the highest expected payoff within this class:

$$
\begin{aligned}
& \mathbb{E}_{\pi_{2}}\left(r_{y}\right) \\
= & \mathbb{E}_{\pi_{2}}\left(\hat{v}\left(\mu_{y^{\prime}}\right)\right)-\Phi_{\pi_{2}}=\operatorname{Pr}\left(h^{\prime}\right) v\left(\mu_{h^{\prime}}, a=A\right)-\left(1-\mu_{0}\right)\left(1-\lambda_{b, 2}\right) F \\
= & \mu_{0}\left(B+s N_{g}\right)+\mu_{0} \frac{1-\bar{\mu}_{2}}{\bar{\mu}_{2}}\left(B+s N_{b}\right)-\left(1-\mu_{0}\right)\left(1-\frac{1}{1-\delta}\left(\frac{1-\bar{\mu}_{2}}{\bar{\mu}_{2}} \frac{\mu_{0}}{1-\mu_{0}}-\delta\right)\right) F
\end{aligned}
$$

3. Systems that induce inspection upon seeing a $h^{\prime}$-signal $\left(\chi_{h^{\prime}}=0\right.$ and $\bar{\mu}_{1} \leqslant$ $\left.\mu_{h^{\prime}}<\bar{\mu}_{2}\right)$

If in equilibrium, the investor inspects upon receiving a $h^{\prime}$-signal, then the manager's expected payoff is:

$$
V_{\delta}^{m}\left(\mu_{0}\right) \leqslant \operatorname{Pr}\left(h^{\prime}\right) \hat{v}\left(\mu_{h^{\prime}}\right)+\operatorname{Pr}\left(l^{\prime}\right) \hat{v}\left(\mu_{l^{\prime}}\right) \leqslant \mu_{0}\left(B+s N_{g}\right)
$$

Thus, under this type of system, the mis-aligned manager's expected payoff is always lower than under System $\pi_{1}$, and thus this type of system is never optimal. As a result, when $\mu_{0}<\bar{\mu}_{1}$, the optimal system is either System $\pi_{1}$ or $\pi_{2}$, with $\lambda_{g}=1$. 


\section{A.16 Proof of Proposition 4}

Proof. The proof of Proposition 4 utilizes Lemma A1, A2, and A4. Lemma A4 shows that when $m<\bar{m}$ and $\mu_{0}<\bar{\mu}_{1}$, the mis-aligned manager's optimal system is either System $\pi_{1}$ or $\pi_{2}$.

1. If $\delta>\frac{\mu_{0}}{1-\mu_{0}} \frac{1-\bar{\mu}_{2}}{\bar{\mu}_{2}}$, the optimal system is $\pi_{1}$, since under $\pi_{2}$ the investor has $\mu_{h^{\prime}}\left(\phi_{l}\right)<\bar{\mu}_{2}$ regardless of $\phi_{l}$, and will not approve outright.

2. If $\delta<\frac{\mu_{0}}{1-\mu_{0}} \frac{1-\bar{\mu}_{2}}{\bar{\mu}_{2}}$, both $\pi_{1}$ and $\pi_{2}$ are potentially optimal; since both $\mathbb{E}_{\pi_{1}}\left(r_{y}\right), \mathbb{E}_{\pi_{2}}\left(r_{y}\right)$ are linear in $F$, I obtain the cutoff $\bar{f}_{2}$, above which the manager is better off with System $\pi_{1}: \bar{f}_{2}=\frac{\frac{1-\bar{\mu}_{2}}{\bar{\mu}_{2}} \delta\left(B+s N_{b}\right)}{\frac{1-\bar{\mu}_{2}}{\bar{\mu}_{2}}+\frac{1-\mu_{0}}{\mu_{0}}\left(1-\lambda_{b, 2}\right) \delta}$, where $\lambda_{b, 2}=\frac{1}{1-\delta}\left(\frac{1-\bar{\mu}_{2}}{\bar{\mu}_{2}} \frac{\mu_{0}}{1-\mu_{0}}-\delta\right)$.

(a) If $F<\bar{f}_{2}, \mathbb{E}_{\pi_{2}}\left(r_{y}\right) \geqslant \mathbb{E}_{\pi_{1}}\left(r_{y}\right)$. The optimal system is $\pi_{2}$ with $\lambda_{g}=1$, $\lambda_{b, 2}=\frac{1}{1-\delta}\left(\frac{1-\bar{\mu}_{2}}{\bar{\mu}_{2}} \frac{\mu_{0}}{1-\mu_{0}}-\delta\right)$. In equilibrium, the $l$-signal manager manipulates with probability $\phi_{l}=1$. Despite the manipulation, the investor's posterior $\mu_{h^{\prime}}\left(\phi_{l}=1\right)=\bar{\mu}_{2}$, and she will approve outright upon seeing a $h^{\prime}$-signal.

(b) If $F>\bar{f}_{2}, \mathbb{E}_{\pi_{1}}\left(r_{y}\right) \geqslant \mathbb{E}_{\pi_{2}}\left(r_{y}\right)$. In equilibrium, the $l$-signal manager manipulates with probability $\phi_{l}=0$, and the investor's posterior has $\mu_{h^{\prime}}\left(\phi_{l}=1\right)=\bar{\mu}_{2}$. However, the investor approves upon seeing a $h^{\prime}$-signal with probability $\chi_{h^{\prime}}=\frac{F}{\delta\left(B+s N_{b}\right)}$.

\section{A.17 Proof of Corollary 4}

Proof. This corollary has two parts. 
1. Note that $\mathbb{E}_{\pi_{1}}\left(r_{y}\right), \mathbb{E}_{\pi_{2}}\left(r_{y}\right)$ decrease in $\delta$ :

$$
\begin{aligned}
\frac{\partial \mathbb{E}_{\pi_{1}}\left(r_{y}\right)}{\partial \delta} & =-\frac{F}{\delta^{2}\left(B+s N_{b}\right)}\left[\mu_{0}\left(B+s N_{g}\right)+\mu_{0} \frac{1-\tilde{\mu}}{\tilde{\mu}}\left(B+s N_{b}\right)\right]<0 \\
\frac{\partial \mathbb{E}_{\pi_{2}}\left(r_{y}\right)}{\partial \delta} & =\left(1-\mu_{0}\right) \frac{1}{(1-\delta)^{2}}\left(\frac{1-\tilde{\mu}}{\tilde{\mu}} \frac{\mu_{0}}{1-\mu_{0}}-1\right) F<0
\end{aligned}
$$

The second inequality uses $\mu_{0}<\tilde{\mu}$. Thus, $V_{\delta}^{m}\left(\mu_{0}\right)=\max \left\{\mathbb{E}_{\pi_{1}}\left(r_{y}\right), \mathbb{E}_{\pi_{2}}\left(r_{y}\right)\right\}$ decreases in $\delta$.

2. When $\delta<\frac{\mu_{0}}{1-\mu_{0}} \frac{1-\tilde{\mu}}{\tilde{\mu}}$, System $\pi_{2}$ is potentially optimal. When $F$ is sufficiently small (i.e., $F<\bar{f}_{2}$ in the $m<\bar{m}$ case and $F<\bar{f}_{1}$ in the $m \geqslant \bar{m}$ case), the optimal system is $\pi_{2}$. Since $\mathbb{E}_{\pi_{2}}\left(r_{y}\left(\mu_{0}\right)\right)$ decreases in $F, V_{\delta}^{m}\left(\mu_{0}\right)=$ $\max \left\{\mathbb{E}_{\pi_{1}}\left(r_{y}\right), \mathbb{E}_{\pi_{2}}\left(r_{y}\right)\right\}$ decreases in $F$ when $F$ is small. When $F$ is sufficiently large (i.e., $F>\bar{f}_{2}$ in the $m<\bar{m}$ case, and $F>\bar{f}_{1}$ in the $m \geqslant \bar{m}$ case), the optimal system is $\pi_{1}$. Since $\mathbb{E}_{\pi_{1}}\left(r_{y}\left(\mu_{0}\right)\right)$ increases in $F, V_{\delta}^{m}\left(\mu_{0}\right)$ increases in $F$ when $F$ is sufficiently large.

\section{A.18 Proof of Corollary 5}

Proof. Note that the investor's posteriors in the manipulation case are $\mu_{h^{\prime}}=\tilde{\mu}$ and $\mu_{l^{\prime}}=0$, the same as the posteriors in the no-manipulation case. Thus, as $m$ decreases, the post-manipulation signal $y^{\prime}$ becomes less liberal and more informative.

\section{A.19 Proof of Corollary 6}

Proof. Under System $\pi_{1}$, over-investment exists since bad projects receive a $h^{\prime}$-signal with probability $\lambda_{b}=\frac{1-\tilde{\mu}}{\tilde{\mu}} \frac{\mu_{0}}{1-\mu_{0}}$, and conditional on receiving a $h^{\prime}$-signal, are approved outright with probability $\chi_{h^{\prime}}=\frac{F}{\delta\left(B+s N_{b}\right)}$ and inspected with probability $1-\chi_{h^{\prime}}$. System $\pi_{2}$ leads to over-investment since bad projects receive a $h^{\prime}$-signal with probability 
$\frac{1-\tilde{\mu}}{\tilde{\mu}} \frac{\mu_{0}}{1-\mu_{0}}$ and are approved with certainty. As $\delta$ increases, the optimal system changes from $\pi_{2}$ to $\pi_{1}$ and the probability of inspection $\left(1-\chi_{h^{\prime}}\right)$ increases. As a result, overinvestment deceases in $\delta$. 


\title{
Appendix B
}

\author{
Summary of Results
}


Table B.1: The Mis-aligned Manager's Optimal System in the Basic Model

\begin{tabular}{ccc} 
Prior & High inspection cost $(m>\bar{m})$ & Low inspection cost $(m<\bar{m})$ \\
\hline Optimistic & Uninformative & Uninformative \\
$\left(\mu_{0} \geqslant \max \left(\bar{\mu}, \bar{\mu}_{2}\right)\right)$ & $\mu_{h}=\mu_{0}, \mu_{l}=\mu_{0}$ & $\mu_{h}=\mu_{0}, \mu_{l}=\mu_{0}$ \\
& $\lambda_{g}=\lambda_{b}$ & $\lambda_{g}=\lambda_{b}$ \\
\hline Intermediate & $\mathrm{N} / \mathrm{A}$ & Partially Revealing, Positive Bias \\
$\left(\bar{\mu}_{1}<\mu_{0}<\bar{\mu}_{2}\right)$ & $\begin{array}{c}\mu_{h}=\bar{\mu}_{2}, \mu_{l}=0 \\
\end{array}$ & $\lambda_{g}=1, \lambda_{b}=\frac{\mu_{0}}{1-\mu_{0}} \frac{1-\bar{\mu}_{2}}{\bar{\mu}_{2}}$ \\
\hline Pessimistic & Partially Revealing, Positive Bias & Partially Revealing, Positive Bias \\
$\left(\mu_{0}<\min \left(\bar{\mu}, \bar{\mu}_{1}\right)\right)$ & $\mu_{h}=\bar{\mu}, \mu_{l}=0$ & $\mu_{h}=\bar{\mu}_{2}, \mu_{l}=0$ \\
& $\lambda_{g}=1, \lambda_{b}=\frac{\mu_{0}}{1-\mu_{0}} \frac{1-\bar{\mu}}{\bar{\mu}}$ & $\lambda_{g}=1, \lambda_{b}=\frac{\mu_{0}}{1-\mu_{0}} \frac{1-\bar{\mu}_{2}}{\bar{\mu}_{2}}$
\end{tabular}


Table B.2: The Well-aligned Manager's Optimal System in the Basic Model

\begin{tabular}{ccc} 
Prior & High inspection cost $(m>\bar{m})$ & Low inspection cost $(m<\bar{m})$ \\
\hline Optimistic & Fully Revealing & Partially Revealing, Negative Bias \\
$\left(\mu_{0} \geqslant \max \left(\bar{\mu}, \bar{\mu}_{2}\right)\right)$ & $\mu_{h}=1, \mu_{l}=0$ & $\mu_{h}=1, \mu_{l}=\bar{\mu}_{2}$ \\
& $\lambda_{g}=1, \lambda_{b}=0$ & $\lambda_{g}=\frac{\mu_{0}-\bar{\mu}_{2}}{\mu_{0}\left(1-\bar{\mu}_{2}\right)}, \lambda_{b}=0$ \\
\hline Intermediate & $\mathrm{N} / \mathrm{A}$ & Uninformative \\
$\left(\bar{\mu}_{1}<\mu_{0}<\bar{\mu}_{2}\right)$ & $\mu_{h}=\mu_{0}, \mu_{l}=\mu_{0}$ \\
& $\lambda_{g}=\lambda_{b}$ \\
\hline Pessimistic & Fully Revealing & Partially Revealing, Positive Bias \\
$\left(\mu_{0}<\min \left(\bar{\mu}, \bar{\mu}_{1}\right)\right)$ & $\mu_{h}=1, \mu_{l}=0$ & $\mu_{h}=\bar{\mu}_{1}, \mu_{l}=0$ \\
& $\lambda_{g}=1, \lambda_{b}=0$ & $\lambda_{g}=1, \lambda_{b}=\frac{\mu_{0}}{1-\mu_{0}} \frac{1-\bar{\mu}_{1}}{\bar{\mu}_{1}}$ \\
\hline
\end{tabular}


Table B.3: The Mis-aligned Manager's Optimal System in the Extended Model

\begin{tabular}{ccc} 
Prior & High inspection cost $(m>\bar{m})$ & Low inspection cost $(m<\bar{m})$ \\
\hline Optimistic & Same as in Table B.1 & Same as in Table B.1 \\
$\left(\mu_{0} \geqslant \max \left(\bar{\mu}, \bar{\mu}_{2}\right)\right)$ & $\mathrm{N} / \mathrm{A}$ & $\begin{array}{c}\text { Partially Revealing, Positive Bias } \\
\mu_{h^{\prime}}=\bar{\mu}_{2}, \mu_{l^{\prime}}=0 \text { or } \mu_{l^{\prime}} \in\left(\bar{\mu}_{1}, \mu_{0}\right) \\
\chi_{h^{\prime}}=1 \text { or } \frac{F}{\delta\left(1-\mu_{l^{\prime}}\right)\left(B+s N_{b}\right)}\end{array}$ \\
\hline Intermediate & $\begin{array}{c}\text { Partially Revealing, Positive Bias } \\
\left(\bar{\mu}_{1}<\mu_{0}<\bar{\mu}_{2}\right)\end{array}$ & $\begin{array}{c}\mu_{h^{\prime}}=\bar{\mu}_{2}, \mu_{l^{\prime}}=0 \\
\chi_{h^{\prime}}=1 \text { or } \frac{F}{\delta\left(B+s N_{b}\right)}\end{array}$ \\
\hline Pessimistic & Partially Revealing, Positive Bias \\
$\left(\mu_{0}<\min \left(\bar{\mu}, \bar{\mu}_{1}\right)\right)$ & $\mu_{h^{\prime}}=\bar{\mu}, \mu_{l^{\prime}}=0$ & $F$ \\
& $\chi_{h^{\prime}}=1$ or $\frac{F}{\delta\left(B+s N_{b}\right)}$ &
\end{tabular}


Table B.4: The Well-aligned Manager's Optimal System in the Extended Model

\begin{tabular}{ccc} 
Prior & High inspection cost $(m>\bar{m})$ & Low inspection cost $(m<\bar{m})$ \\
\hline $\begin{array}{c}\text { Optimistic } \\
\left(\mu_{0} \geqslant \max \left(\bar{\mu}, \bar{\mu}_{2}\right)\right)\end{array}$ & Same as in Table B.2 & Same as in Table B.2 \\
\hline $\begin{array}{c}\text { Intermediate } \\
\left(\bar{\mu}_{1}<\mu_{0}<\bar{\mu}_{2}\right)\end{array}$ & Same as in Table B.2 \\
\hline $\begin{array}{c}\text { Pessimistic } \\
\left(\mu_{0}<\min \left(\bar{\mu}, \bar{\mu}_{1}\right)\right)\end{array}$ & Same as in Table B.2 & Same as in Table B.2 \\
& &
\end{tabular}




\section{Bibliography}

Aboody, D. and Kasznik, R. (2000), "CEO stock option awards and the timing of corporate voluntary disclosures," Journal of Accounting and Economics, 29, 73100.

Armstrong, C., Taylor, D., and Verrecchia, R. (Forthcoming), "Asymmetric Reporting," Journal of Financial Reporting.

Arya, A., Glover, J., and Sunder, S. (1998), "Earnings Management and the Revelation Principle," Review of Accounting Studies, 3, 7-34.

Bagnoli, M. and Watts, S. (2005), "Conservative Accounting Choices," Management Science, 51, 786-801.

Balakrishnan, K., Billings, M., Kelly, B., and Ljungqvist, A. (2014), "Shaping Liquidity: On the Causal Effects of Voluntary Disclosure," The Journal of Finance, $69,2237-2278$.

Ball, R., Robin, A., and Wu, J. (2003), "Incentives Versus Standards: Properties of Accounting Income in Four East Asian countries," Journal of Accounting and Economics, 36, 235-270.

Barton, J. and Simko, P. (2002), "The Balance Sheet as an Earnings Management Constraint," The Accounting Review, 77, 1-27.

Basu, S. (1997), "The conservatism principle and the asymmetric timeliness of earnings," Journal of Accounting and Economics, 24, 3-37.

Bertomeu, J. and Cheynel, E. (2015), "Asset Measurement in Imperfect Credit Markets," Journal of Accounting Research, 53, 965-984.

Bertomeu, J., Beyer, A., and Dye, R. A. (2011), "Capital Structure, Cost of Capital, and Voluntary Disclosures," The Accounting Review, 86, 857-886.

Beyer, A. and Guttman, I. (2012), "Voluntary Disclosure, Manipulation, and Real Effects," Journal of Accounting Research, 50, 1141-1177.

Beyer, A., Guttman, I., and Marinovic, I. (2014), "Optimal Contracts with Performance Manipulation," Journal of Accounting Research, 52, 817-847. 
Biddle, G., Hilary, G., and Verdi, R. (2009), "How does Financial Reporting Quality Relate to Investment Efficiency?" Journal of Accounting and Economics, 48, 112131.

Burgstahler, D. and Dichev, I. (1997), "Earnings Management to Avoid Earnings Decreases and Losses," Journal of Accounting and Economics, 24, 99-126.

Caskey, J. and Hughes, J. (2011), "Assessing the impact of alternative fair value measures on the efficiency of project selection and continuation," The Accounting Review, 87, 483-512.

Chen, Q. and Vashishtha, R. (2015), "The Effects of Bank Mergers on Corporate Information Disclosure," Working Paper.

Chen, Q., Hemmer, T., and Zhang, Y. (2007), "On the relation between conservatism in accounting standards and incentives for earnings management," Journal of Accounting Research, 45, 541-565.

Chen, Q., Lewis, T., Schipper, K., and Zhang, Y. (F), "Uniform vs. Discretionary Regimes in Reporting Information with Unverifiable Precision and a Coordination Role," Journal of Accounting Research.

Dechow, P., Ge, W., and Schrand, C. (2010), "Understanding earnings quality: A review of the proxies, their determinants and their consequences," Journal of Accounting and Economics, 50, 344-401.

DeFond, M. and Zhang, J. (2014), "A review of archival auditing research," Journal of Accounting and Economics, 58, 275-326.

DeFond, M., Erkens, D., and Zhang, J. (2015), "Does PSM Really Eliminate the Big N Audit Quality Effect?" Working Paper.

Demski, J. and Feltham, G. (1994), "Market response to financial reports," Journal of Accounting and Economics, 17, 3-40.

Diamond, D. (1985), "Optimal Release of Information By Firms," The Journal of Finance, 40, 1071-1094.

Diamond, D. and Verrecchia, R. (1991), "Disclosure, Liquidity, and the Cost of Capital," The Journal of Finance, 46, 1325-1359.

Dutta, S. and Gigler, F. (2002), "The Effect of Earnings Forecasts on Earnings Management," Journal of Accounting Research, 40, 631-655.

Dye, R. (1985), "Disclosure of Nonproprietary Information," Journal of Accounting Research, 23, 123-145. 
Dye, R. and Sridhar, S. (2002), "Resource Allocation Effects of Price Reactions to Disclosures," Contemporary Accounting Research, 19, 385-410.

FASB (2010), "Statement of Financial Accounting Concepts No. 8: Conceptual Framework for Financial Reporting," .

Francis, J. and Martin, X. (2010), "Acquisition profitability and timely loss recognition," Journal of Accounting and Economics, 49, 161-178.

Francis, J., Schipper, K., and Vincent, L. (2002a), "Earnings announcements and competing information," Journal of Accounting and Economics, 33, 313-342.

Francis, J., Schipper, K., and Vincent, L. (2002b), "Expanded Disclosures and the Increased Usefulness of Earnings Announcements," The Accounting Review, 77, $515-546$.

Friedman, H., Hughes, J., and Michaeli, B. (2015), "The Impact of Discretionary Disclosure on Financial Reporting Systems: An Extension of Bayesian Persuasion," Working Paper.

Gao, P. (2013), "A measurement approach to conservatism and earnings management," Journal of Accounting and Economics, 55, 251-268.

Gao, P. and Liang, P. (2013), "Informational Feedback, Adverse Selection, and Optimal Disclosure Policy," Journal of Accounting Research, 51, 1133-1158.

Gentzkow, M. and Kamenica, E. (2014), "Costly Persuasion," American Economic Review, 104, 457-62.

Gigler, F. and Hemmer, T. (2001), "Conservatism, optimal disclosure policy, and the timeliness of financial reports," The Accounting Review, 76, 471-493.

Gigler, F., Kanodia, C., Sapra, H., and Venugopalan, R. (2009), "Accounting Conservatism and the Efficiency of Debt Contracts," Journal of Accounting Research, $47,767-797$.

Givoly, D. and Hayn, C. (2000), "The changing time-series properties of earnings, cash flows and accruals: Has financial reporting become more conservative?" Journal of Accounting and Economics, 29, 287-320.

Goex, R. and Wagenhofer, A. (2009), "Optimal impairment rules," Journal of Accounting and Economics, 48, 2-16.

Healy, P. and Palepu, K. (2001), "Information asymmetry, corporate disclosure, and the capital markets: A review of the empirical disclosure literature," Journal of Accounting and Economics, 31, 405-440. 
Hemmer, T. and Labro, E. (2008), "On the Optimal Relation between the Properties of Managerial and Financial Reporting Systems," Journal of Accounting Research, 46, 1209-1240.

Hutton, A., Lee, L., and Shu, S. (2012), "Do Managers Always Know Better? The Relative Accuracy of Management and Analyst Forecasts," Journal of Accounting Research, 50, 1217-1244.

Jayaraman, S. (2008), "Earnings Volatility, Cash Flow Volatility, and Informed Trading," Journal of Accounting Research, 46, 809-851.

Jiang, X. (2012), "Accounting Conservatism and Debt Contract Efficiency with Soft Information," Working Paper.

Jiang, X. and Xin, B. (2015), "Accounting Discretion and Informativeness of Voluntary Disclosure," Working Paper.

Jiang, X. and Yang, M. (2015), "Properties of Optimal Accounting Rules in a Signalling Game," Working Paper.

Jung, W. and Kwon, Y. (1988), "Disclosure When the Market Is Unsure of Information Endowment of Managers," Journal of Accounting Research, 26, 146-153.

Kamenica, E. and Gentzkow, M. (2011), "Bayesian Persuasion," American Economic Review, 101, 2590-2615.

Kanodia, C. and Lee, D. (1998), "Investment and Disclosure: The Disciplinary Role of Periodic Performance Reports," Journal of Accounting Research, 36, 33-55.

Kanodia, C., Sapra, H., and Venugopalan, R. (2004), "Should Intangibles Be Measured: What Are the Economic Trade-Offs?" Journal of Accounting Research, 42, 89-120.

Kanodia, C., Singh, R., and Spero, A. (2005), "Imprecision in Accounting Measurement: Can It Be Value Enhancing?" Journal of Accounting Research, 43, 487-519.

Kasznik, R. and Lev, B. (1995), "To Warn or Not to Warn: Management Disclosures in the Face of an Earnings Surprise," The Accounting Review, 70, 113-134.

Kim, O. and Verrecchia, R. (1997), "Pre-announcement and event-period private information," Journal of Accounting and Economics, 24, 395-419.

Kothari, S., Shu, S., and Wysocki, P. (2009), "Do Managers Withhold Bad News?" Journal of Accounting Research, 47, 241-276.

Kumar, P., Langberg, N., and Sivaramakrishnan, K. (2012), "Voluntary Disclosures, Corporate Control, and Investment," Journal of Accounting Research, 50, 10411076 . 
Laux, V. (2014), "Pay Convexity, Earnings Manipulation, and Project Continuation," The Accounting Review, 89, 2233-2259.

Lawrence, A., Minutti-Meza, M., and Zhang, P. (2011), "Can Big 4 versus Non-Big 4 Differences in Audit-Quality Proxies Be Attributed to Client Characteristics?" The Accounting Review, 86, 259-286.

Leuz, C., Nanda, D., and Wysocki, P. (2003), "Earnings Management and Investor Protection: An International Comparison," Journal of Financial Economics, 69, 505-527.

Lev, B. and Zarowin, P. (1999), "The Boundaries of Financial Reporting and How to Extend Them," Journal of Accounting Research, 37, 353-385.

Liu, C. and Ryan, S. (2006), "Income Smoothing over the Business Cycle: Changes in Banks' Coordinated Management of Provisions for Loan Losses and Loan ChargeOffs from the Pre-1990 Bust to the 1990s Boom," The Accounting Review, 81, 421-441.

Magee, R. (2001), "Discussion of "Contracting theory and accounting"," Journal of Accounting and Economics, 32, 89-96.

McNichols, M. and Trueman, B. (1994), "Public disclosure, private information collection, and short-term trading," Journal of Accounting and Economics, 17, 69-94.

Michaeli, B. (2014), "Divide and Inform: Rationing Information to Facilitate Persuasion," Working Paper.

Povel, P., Singh, R., and Winton, A. (2007), "Booms, Busts, and Fraud," Review of Financial Studies, 20, 1219-1254.

Roychowdhury, S. (2010), "Discussion of "Acquisition profitability and timely loss recognition" by J. Francis and X. Martin," Journal of Accounting and Economics, 49, 179-183.

Sapra, H. (2002), "Do Mandatory Hedge Disclosures Discourage or Encourage Excessive Speculation?" Journal of Accounting Research, 40, 933-964.

Sims, C. (2006), "Rational inattention: Beyond the linear-quadratic case," American Economic Review, 96, 158-163.

Skinner, D. (1994), "Why Firms Voluntarily Disclose Bad News," Journal of Accounting Research, 32, 38-60.

Tucker, J. and Zarowin, P. (2006), "Does Income Smoothing Improve Earnings Informativeness?" The Accounting Review, 81, 251-270. 
Verrecchia, R. (1983), "Discretionary disclosure," Journal of Accounting and Economics, 5, 179-194.

Zuo, L. (2013), "The Informational Feedback Effect of Stock Prices on Management Forecasts," MIT Sloan Doctoral Dissertation. 


\section{Biography}

Zeqiong Huang graduated from Tsinghua University in 2008 with a bachelor's degree in Management (Accounting). She also earned a master's degree in Economics from Duke University in 2010. She will receive her Ph.D. in Business Administration (Accounting) from Duke University in 2016. She will join the School of Management at Yale University in July, 2016. 